\title{
THE FEASIBILITY AND APPLICATION OF OBSERVING SMALL LEO SATELLITES WITH AMATEUR TELESCOPES
}

\author{
A Thesis \\ Presented to \\ the Faculty of California Polytechnic State University \\ San Luis Obispo
}

\author{
In Partial Fulfillment \\ of the Requirements for the Degree \\ Master of Science in Aerospace Engineering
}

by

Brock Schmalzel

August 2013 
(C) 2013

Brock Schmalzel

ALL RIGHTS RESERVED 
COMMITTEE MEMBERSHIP

TITLE:

The Feasibility and Application of Observing Small LEO Satellites with Amateur Telescopes

AUTHOR: $\quad$ Brock Schmalzel

DATE SUBMITTED: $\quad$ August 2013

COMMITTEE CHAIR: Dr. Kira Abercromby, Assistant Professor, Department of Aerospace Engineering

COMMITTEE MEMBER: Dr. Russell M. Genet, Research Scholar in Residence

COMMITTEE MEMBER: Dr. John Keller, Assistant Professor, Department of Physics

COMmitTeE MEMBER: Dr. Jordi Puig-Suari, Professor, Department of Aerospace Engineering 


\begin{abstract}
The Feasibility and Application of Observing Small LEO Satellites with Amateur Telescopes

Brock Schmalzel
\end{abstract}

This thesis demonstrates that any individual can provide relevant observational data to further research efforts within the Aerospace community, through the use of amateur telescopes. A Meade LX200 12 in. telescope and Lumenera Skynyx 2.0 camera were utilized to observe small LEO satellites, using a welldocumented point-and-wait staring method. Over a period of three months, a total of 186 observation attempts were made resulting in 97 successful captures. From the gathered data, three possible aerospace applications were analyzed: validation of a satellite brightness prediction model, angles-only orbit determination including extended Kalman filtering, and temporal error growth in TLE-based orbit propagation. Further investigations include a preliminary optimization using MATLAB's fmincon function (informed by the previous analyses) to determine an optimal telescope size for performing LEO observations. 


\section{ACKNOWLEDGMENTS}

Special thanks is given to my advisor, Dr. Kira Abercromby, for her generous help and persistent encouragement throughout this project. I would also like to thank Dr. Jordi Puig-Suari and Dr. Rob McDonald for their valuable input, and Dr. Russ Genet and Dr. John Kelly - their knowledge and resources proved invaluable to an aerospace engineer disguising himself as an astronomer! Lastly much gratitude is given to my friends and family who offered their advice, love, and support during this project. 


\section{TABLE OF CONTENTS}

LIST OF TABLES viii

LIST OF FIGURES L x

LIST OF ACRONYMS X xii

LIST OF SYMBOLS xiv

1 INTRODUCTION 1

2 BACKGROUND AND LITERATURE REVIEW 3

2.1 Satellite Observation ... . . . . . . . . . . . . 3

2.1.1 Orbit Determination - Astrometry . . . . . . . . . 6

2.1.2 Satellite Apparent Brightness - Photometry . . . . . . . . 9

2.2 Initial Plan to Observe CP5 Cubesat . . . . . . . . . . . . . . . . 12

3 INSTRUMENTATION AND PROCEDURE 13

3.1 Instrumentation . . . . . . . . . . . . . . . . . . . 13

3.1 .1 Observatory ........................ 13

3.1.2 Telescope ....................... 13

3.1.3 Video Camera . . . . . . . . . . . . . . . . . . . 14

3.1.4 Focal Reducer . . . . . . . . . . . . . . . . . 16

3.1.5 Software ..................... 16

3.2 Procedure . . . . . . . . . . . . . . . . . . . . 18

3.2.1 Preparation ..................... 18

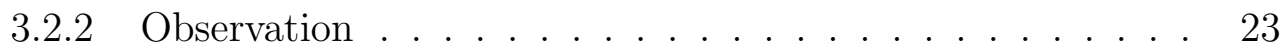

4 ANALYSIS 27

4.1 Review of Images . . . . . . . . . . . . . . . . . . . 27

4.2 Astrometry . . . . . . . . . . . . . . . . . . . 28

4.2.1 Data Reduction . . . . . . . . . . . . . . . . 28

4.2.2 Orbit Determination . . . . . . . . . . . . . 31

4.2.3 Temporal TLE Error Growth . . . . . . . . . . . . . . 32 
4.3 Photometry . . . . . . . . . . . . . . . . . . . 34

4.3 .1 Data Reduction . . . . . . . . . . . . . . . . . . . 34

4.3.2 Magnitude Prediction Model . . . . . . . . . . . . . . . 35

4.4 Telescope Optimization . . . . . . . . . . . . . . . . . . . . . 37

5 RESULTS AND DISCUSSION 41

5.1 Orbit Determination . . . . . . . . . . . . . . . . . 42

5.2 Temporal TLE Error Growth _ . . . . . . . . . . . . . 48

5.3 Magnitude Prediction Model . . . . . . . . . . . . . . . . . 51

5.4 Telescope Optimization . . . . . . . . . . . . . . . . . . . 53

6 CONCLUSIONS $\quad 59$

6.1 Recommendations for Future Research . . . . . . . . . . 60

7 APPENDICES 65

7.1 Selected Image Sets \& Raw Data . . . . . . . . . . . 65

7.2 Lessons Learned . . . . . . . . . . . . . . . . . . . . . . 75

7.3 Custom List of Satellite Targets _ . . . . . . . . . . . 77

7.4 Explanation of Selected MATLAB Codes . . . . . . . . . . 79

7.5 Extended Kalman Filter . . . . . . . . . . . . . . . . . 97

7.6 Error Analysis . . . . . . . . . . . . . . . . . . . . . 100

$\begin{array}{ll}\text { BIBLIOGRAPHY } & 101\end{array}$ 


\section{LIST OF TABLES}

2.1 Predicted apparent magnitudes of the CP5 cubesat . . . . . . . . 12

3.1 Meade LX200 12" Telescope Specifications . . . . . . . . . . . . . 14

3.2 Lumenera SKYnyx2-0M Camera Specifications . . . . . . . . . . . 16

3.3 Information Archived from Preparation . . . . . . . . . . . . . 23

4.1 Details of the Fmincon Telescope Optimizer . . . . . . . . . . . 38

4.2 Fmincon Optimizer Constraints . . . . . . . . . . . . . . . . 40

5.1 Single Pass Orbit Determination Results . . . . . . . . . . . . 43

5.2 Single Pass Orbit Determination Results cont. . . . . . . . . . . . 44

5.3 Correlation between Determined COE and State Vector Errors, and Observational Parameters . . . . . . . . . . . . . . 45

5.4 Comparison of Performance Times for Orbit Determination Methods 46

5.5 Double Pass Orbit Determination Results . . . . . . . . . . . . . . 47

5.6 Mean Accuracy of the Orbit Determination Methods . . . . . . . 47

5.7 Circularization of Orbit Determination Results with use of Fmincon 48

5.8 Telescope Optimization for three test cases . . . . . . . . . . 55

5.9 Commercial Telescopes matched to Optimization Results . . . . . 58

7.1 Observational Results - Astrometry Data (Observational data including the predicted target (Tar.) and actual determined (Det.) RA/Dec angles $\ldots \ldots \ldots . \ldots . \ldots 65$

7.2 Observational Results - Photometry Data Part 1 (Observational data relevant to photometric analysis, where D1 and D2 are dimensions of each satellite in meters (if listed as 0 , dimension was not known), and TLE is the age of the reference TLE) . . . . . 71

7.3 Observational Results - Photometry Data Part 2 (Observational data relevant to photometric analysis-listed are the apparent magnitudes from each source, including the companion star) . . . . . 73 
7.4 Additional Single Pass Orbit Determination Results (Comparison of COE's generated by angles-only orbit determination method, to COE's gathered from propagation-source TLE's) . . . . . . . . . . 74

7.5 Satellites of Interest List _ . . . . . . . . . . . . . . . 79

7.6 Pixel Selection Errors determined by Monte-Carlo Method . . . . 100 


\section{LIST OF FIGURES}

1.1 Visualization of satellites and debris actively tracked in LEO . . . 2

2.1 Echo 1 Spacecraft launched on August 12, 1960 . . . . . . . . 4

2.2 Visualization of Right Ascension and Declination within the Celestial Sphere . . . . . . . . . . . . . . 8

2.3 Radial, transverse, and cross-track residuals for Stella satellite over 7 days . . . . . . . . . . . . . . . . . . . . 9 9

2.4 Least squares fitted polynomial for standard deviation of radial, transverse, and cross-track residuals for Stella satellite . . . . . . 10

2.5 Telescope limiting magnitude for a staring system . . . . . . . . . 11

3.1 Cal Poly Dome Observatory . . . . . . . . . . . . . . . 14

3.2 Meade LX200 Telescope . . . . . . . . . . . . . . . . . . . . . . . 15

3.3 Lumenera SKYnyx2-0M Astrophotography Camera . . . . . . . . 15

3.4 Celestron $\mathrm{f} / 3.3$ Focal Reducer . . . . . . . . . . . . . . . . 17

3.5 Full camera setup/camera attached to back end of Meade telescope 17

3.6 Sample Output from N2YO website . . . . . . . . . . . . . . . . . 19

3.7 Output of VizieR Service for List of Targets Query . . . . . . . . 21

3.8 Active Interface of Lucam Recorder . . . . . . . . . . . . . . . 25

3.9 Step-by-step Observation Procedure. Includes information sent along each step: satellite Two-Line Element Sets (TLE), satellite apparent magnitude prediction (Mag), time and duration of satellite pass (Time), azimuth and elevation angles (Azi/Elev), right ascension and declination angles (RA/Dec), and companion star details (Star). . . . . . . . . . . . . . . 26

4.1 Visualization of Pixel-to-RA/Dec Transformation (Pictured top: Satellite movement from position 1 to 2 (red), separation between star and satellite position 1 (green), and frame axes (yellow). Pictured bottom: Predicted direction (blue), actual direction (red), frame rotation (purple), and RA/Dec axes (black)). . . . . . . 30 
5.1 Successfully Observed Satellites listed within STK Scenario . . . . 42

5.2 Cross-Track Error Results . . . . . . . . . . . . . . . . . . . . . . 49

5.3 Along-Track Error Results . . . . . . . . . . . . . . . . 50

5.4 Magnitude Prediction Model Results . . . . . . . . . . . . . . 52

5.5 Magnitude Prediction Model Results - Last 15 Observations . . . 53

5.6 Telescope Optimization - IMS-1, Slow Temporal Growth . . . . 54

5.7 Telescope Optimization - IMS-1, Fast Temporal Growth . . . . . 55

5.8 Telescope Optimization - Full Population, Slow Temporal Growth 56

5.9 Telescope Optimization - Full Population, Fast Temporal Growth 57

7.1 Observation of IMS-1. Images taken of the Indian Remote Sensing satellite, IMS-1, on April 17 ${ }^{\text {th }}, 2013$, at 21:18 PST. At $83 \mathrm{~kg}$, this satellite was the least massive of all objects observed . . . . . . 66

7.2 Observation of Globalstar M035. Images taken of Globalstar M035, on April 20 th, 2013 , at 23:03 PST. At an apparent magnitude of approximately 11.2, this was the dimmest object observed. For the purpose of presentation, every second image from the set was removed, and the brightness and contrast values for the images increased . . . . . . . . . . . . . . . . . . . . 68

7.3 Observation of Globalstar M002. Images taken of Globalstar M002, on March $12^{\text {th }}, 2013$, at 21:41 PST. At an apparent magnitude of approximately 4.93, this was the brightest object observed. Every second image from the set was removed . . . . . . . . . . . . 69

7.4 Observation of Globalstar M058. Images taken of Globalstar M058, on May $10^{\text {th }}, 2013$, at 20:49 PST. This observation had a notably low cross-track error of only $0.21 \mathrm{~km}$ - every second image from the set was removed . . . . . . . . . . . . . . 70

7.5 Altitude vs. Mass for Satellites of Interest List . . . . . . . . . . . 78 


\section{LIST OF ACRONYMS}

$\begin{array}{ll}\text { ACF } & \text { Advanced Coma Free } \\ \text { AER } & \text { Azimuth-Elevation-Range } \\ \text { ATE } & \text { Along-Track Error } \\ \text { CCD } & \text { Charge-Couple Device } \\ \text { COE } & \text { Common Orbital Elements } \\ \text { CTE } & \text { Cross-Track Error } \\ \text { DDW } & \text { Digital Dome Works } \\ \text { EKF } & \text { Extended Kalman Filter } \\ \text { FOV } & \text { Field of View } \\ \text { GEO } & \text { Geostationary Earth Orbit } \\ \text { GUI } & \text { Graphical User Interface } \\ \text { HEO } & \text { Highly Eccentric Orbit } \\ \text { HPOP } & \text { High Precision Orbit Propagator } \\ \text { LEO } & \text { Low Earth Orbit } \\ \text { LS } & \text { Least Squares Differential Corrector } \\ \text { MATLAB Matrix Laboratory } \\ \text { NORAD }\end{array}$


USB Universal Serial Bus 


\section{LIST OF SYMBOLS}

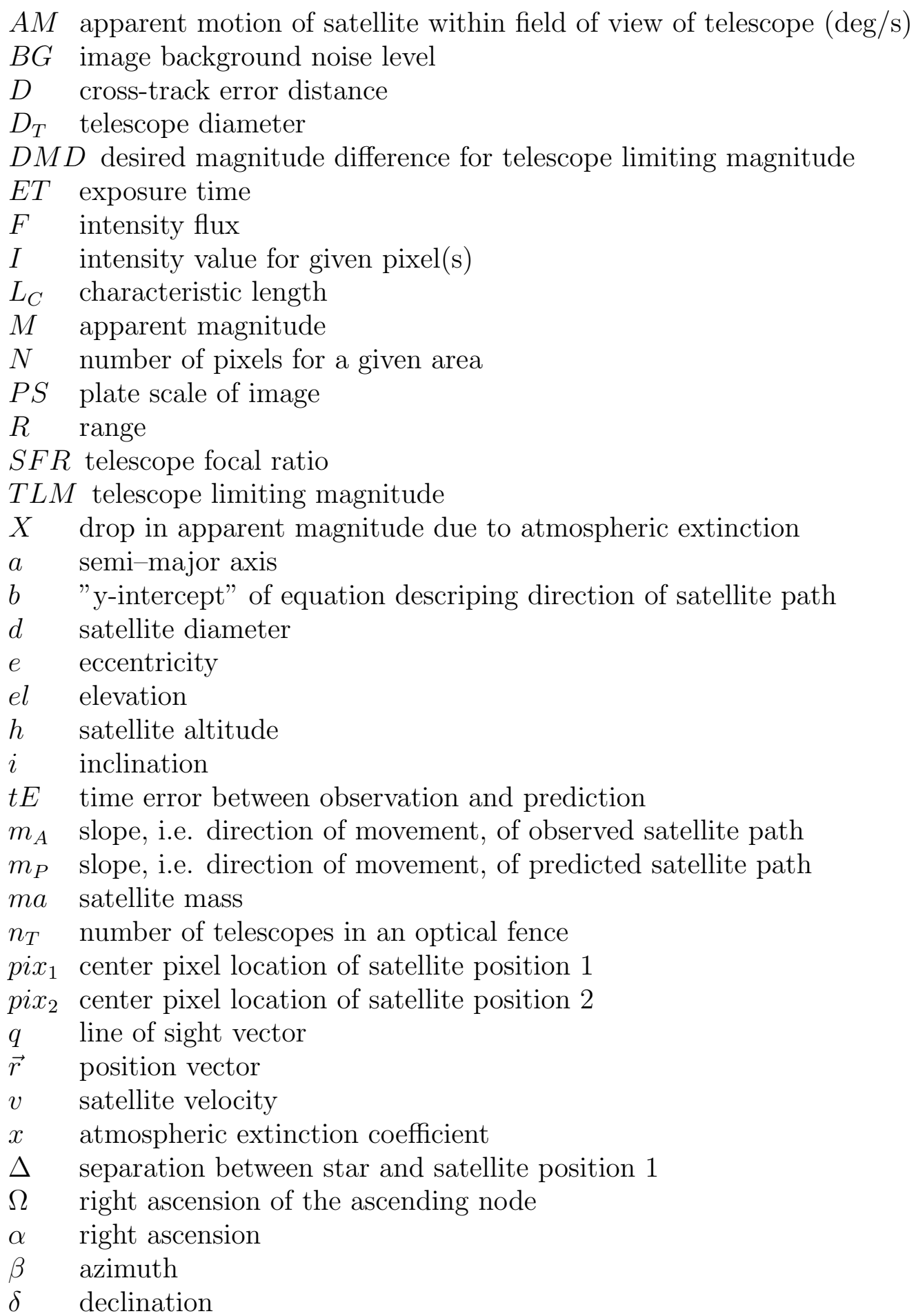


$\mu \quad$ gravitational parameter

$\nu \quad$ true anomaly

$\omega \quad$ argument of perigee

$\theta$ transformation angle from CCD pixel frame to RA/dec frame 


\section{CHAPTER 1}

\section{INTRODUCTION}

As of June $25^{\text {th }}, 2013$, the sum total of spacecraft, rocket bodies, and debris objects tracked by the US Strategic Command's Joint Functional Component Command for Space numbers at 16,824[25]. These satellites are commonly described by, and propagated with the use of, a two-line element set (TLE). TLE's are generated for a specific object from position-velocity state vectors - often created utilizing some form of recursive filtering - and contain information about the orbit of the object. The necessary state vectors for such orbit determination are provided by ground-based observatories through the use of either laser ranging, radar, or optical sensors[29]. Due to the ever-growing number of orbiting objects, established sensor networks such as the US Space Surveillance Network, Russian Space Surveillance System, and International Scientific Optical Network, face increased scheduling constraints. Because of these limits it follows that certain objects are given lower-priority status, e.g. cubesats and other university-built satellites. Where commercial satellites and high-risk debris are frequently targeted by laser ranging and radar observatories, respectively, low-priority satellites may be ideal targets for optical sensors - recently, amateur astronomers have observed many Low Earth Orbit (LEO) satellites, with thirteen observers providing over 21,500 observations in the 2010-11 year[32]. Figure 1.1 displays each of the tracked objects in LEO - the region of space below an altitude of about 2000 $\mathrm{km}$ - represented as white dots.

The primary aim of this paper is to investigate the feasibility and potential 


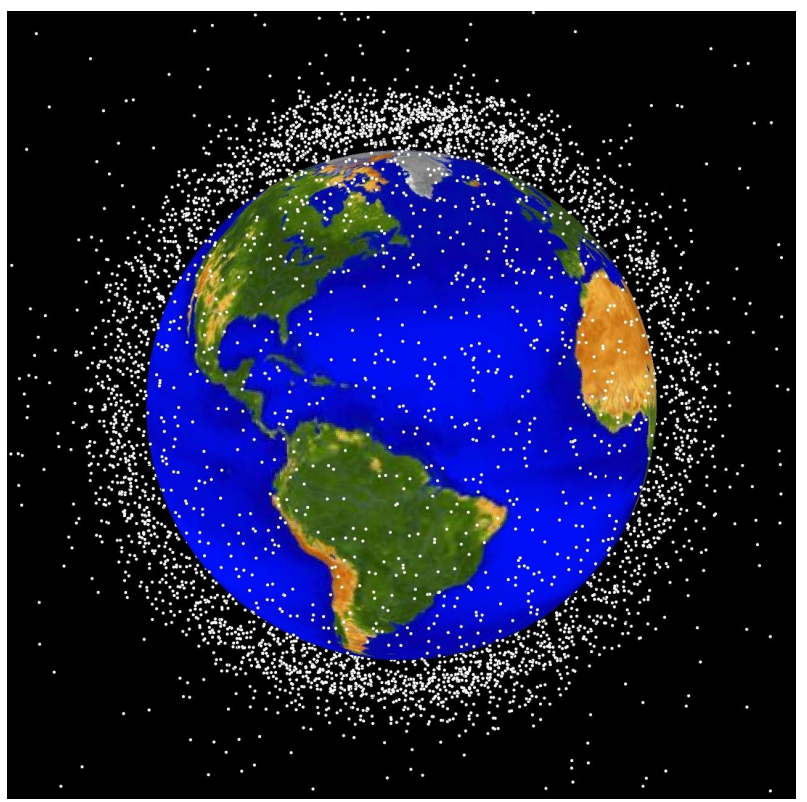

Figure 1.1: Visualization of satellites and debris actively tracked in LEO $[20]$

utility of using amateur, commercial-sized telescopes to observe the aforementioned low-priority, LEO satellites. If such observations can be accomplished by small, cheap telescopes, more operational time can be freed up for other observatories to focus upon targets of higher interest. When considering the potential growth of hazardous debris described by the "Kessler Syndrome" - an exponential increase in orbital debris brought about by collisions not unlike the 2009 collision of Iridium 33 and Cosmos 2251 - this may prove to be an invaluable tool[13]. Additional goals of this paper include the following four investigations that, if successful, could provide data relevant to research within the Aerospace community: preliminary angles-only orbit determination, photometric analysis of satellites for use in validation of a brightness prediction model, astrometrical analysis to provide a measurement of temporal error growth in TLE-based orbit propagation, and an optimization of an ideal telescope for LEO observations (informed by the previous two analyses). 


\section{CHAPTER 2}

\section{BACKGROUND AND LITERATURE REVIEW}

Amidst the search for an engaging and worthwhile thesis topic, advice was received in the form of challenge: find a topic that suits my interests and skills developed from both my aerospace and astronomy classwork. After some deliberation, it was clear that coupling my knowledge of observational astronomy and orbital mechanics would provide an exciting research path: the discipline of satellite observation. In pursuing this discipline - a type of "applied astronomy" - no shortage of academic precursors or historical precedent was found. Presented in this chapter is a review of the research and academic literature found to be influential or informative or both.

\subsection{Satellite Observation}

Telescopic observations of satellites have been performed since the first spacecraft were launched into orbit - the Asiago Astrophysical Observatory observed over a dozen satellites from Sep. 1960 to Aug. 1962, photographed using a moving film technique with a telescope aperture of 0.4-1 m diameter[14]. Satellites such as Echo 1 and the first-generation, Corona spy satellites - at the time known as the "Discoverer" class spacecraft - were targeted by the observatory. A lower limit of 3.5 apparent magnitude was achieved with a stationary camera, whereas a tracking method attained a lower limit of 7-8 magnitude by providing a sustained exposure along a $7^{\circ}$ arc of a satellite's path (see 2.1.2 for explanation 


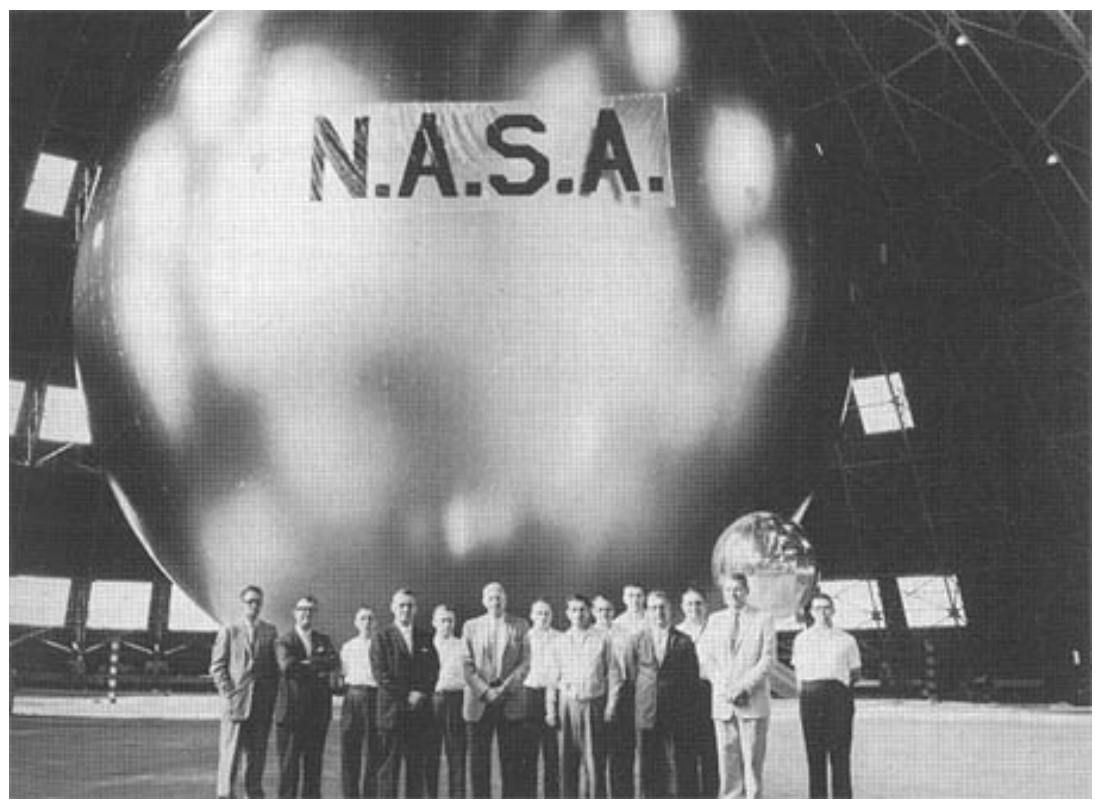

Figure 2.1: Echo 1 Spacecraft launched on August 12, 1960[12]

of apparent magnitude). These restrictive magnitude and exposure limitations were accompanied by additional drawbacks to the system including significant along- and cross-track errors (see 4.2.3). As would be expected, fifty years of technological progress has greatly improved satellite observations.

The improvement of telescopes and telescope control, the development of charge-couple devices (CCD) to replace film, and increased accuracy of orbit propagation methods, have combined to yield satellite observations that are more simple and successful. The $1 \mathrm{~m}$ Zimmerwald optical telescope operated by the University of Bern, routinely searched for debris in Geostationary Earth Orbit (GEO) - altitude of $35,786 \mathrm{~km}$ to provide a geosynchronous orbit - as radar observations are limited to $1 \mathrm{~m}$ or larger objects in GEO[23]. With a one-second exposure, the telescope was able to detect objects up to an apparent magnitude between 17.0 and 19.5 (corresponding to object sizes of 0.58 to $0.18 \mathrm{~m}$, for an albedo of 0.08). For optical observations of LEO objects, the United States Air Force's (USAF) Starfire Optical Range operates a 3.5 m diameter telescope capa- 
ble of seeing objects as small as $\sim 0.24 \mathrm{~m}$ at a range of $\sim 1600 \mathrm{~km}[10]$. For some additional material on the history and development of telescopes and satellites observations, this author suggests Artificial Satellite Observing... and Observational Astronomy[17][3]. More recent research has considered optical observations of LEO objects, which must confront the additional challenge of accounting for the high apparent motion.

Of particular influence was the research of USAF Captain Matthew Schmunk presented in his thesis, "Initial Determination of Low Earth Orbits Using Commercial Telescopes" [24]. Included in Schmunk's motivations for his research was a characterization of commercial telescopes as "inexpensive, mobile, and easily supported", therefore making admittedly less-powerful commercial systems viable for satellite surveillance. With the use of a Meade LX200 GPS telescope and a wide-field digital camera, Schmunk pursued the imaging of satellites for use in angles-only orbit determination. A MATLAB Graphical User Interface (GUI) was developed for satellite target selection, orbital pass and brightness prediction, and tracking - the Simplified General Perturbations-4 (SGP4) model was used for pass prediction. SGP4, initially released in its first form in 1980, uses mean orbital elements (generally from TLE's) with the "incorporation of resonances, third-body forces, atmospheric drag, and other perturbations" to propagate orbits [29]. Due to the telescope's small Field of View (FOV), the main optics were not used - Schmunk utilized a digital camera with a $5.7^{\circ}$ by $4.73^{\circ}$ FOV attached to the side of the telescope[24]. Although Schmunk does not elaborate on this decision, it is safe to assume there may have been significant errors arising from inaccuracies in either the telescope's pointing or the propagation scheme, that would prevent the use of a smaller FOV. At a frame-rate of 5 frames-per-second (fps), the system was limited by an apparent magnitude limit of $\sim 6$. The paper concludes with recommendations for future research including 
the following: improvements in the camera system, an operations optimization problems of 2 or more cameras, and automatic image processing.

Similar papers included USAF Captain Michael E. Graff's, "Development of a Remotely Operated Autonomous Tracking System," concerning a closed-loop control system for active satellite tracking, and United States Navy Commander Michael L. Thrall's, “Orbit Determination of Highly Eccentric Orbits Using a Raven Telescope," regarding the use of the Raven telescope to obtain anglesonly data of Highly Eccentric Orbit (HEO) objects[10][26]. Both theses are of a certain relevance as they concern the use of smaller telescopes (Graff: 10 in. Meade LX200, Thrall: 14.6 in. Raven). Graff joins Schmunk in recommending the development of multiple telescope systems used in parallel for satellite observations. In addition to using a commercial-sized telescope, Thrall utilized Analytical Graphic Incorporated's Systems Tool Kit (STK) program as well as implementing the popular Kalman Filter method for orbit determination. These tools will be revisited in Chapter 4 .

\subsubsection{Orbit Determination - Astrometry}

Observational Astronomy defines astrometry as a "general term...used to describe methods by which the positions of stars may be determined" [3]. But in the case of satellite observations, it is the positions of the satellites that are sought, from which orbital information can be derived. One of the earliest examples of orbit determination was accomplished by Edmond Halley in a famous 1705 publication, which successfully predicted the next appearance of Halley's comet through the use of Isaac Newton's graphical approximation method[29]. Many additional methods have been developed post-Newton, including methods utilizing only pointing angles (optical observations) and those benefiting from supplemen- 
tary range and range-rate information (provided by radar and laser observations). For cases when range data is available, Gauss' solution to Lambert's problem - an analytical solution based upon generalized Euler formulas - or Gibbs' geometrical solution can be used with a minimum of two observation sets. But because this paper concerns itself with optical observations, angles-only methods will be of greater importance.

Pointing angles describe the apparent angular position of an object and are often given as azimuth, $\beta$, and elevation, el, or right ascension, $\alpha$, and declination, $\delta$. Azimuth and elevation describe a position based on the observer's local frame of reference - azimuth measured clockwise from North, elevation measured up from the horizon. Right ascension and declination are independent of the local frame, as they are based upon the equatorial coordinate system-right ascension measured east from the vernal equinox direction, declination measured positive north of the equator and negative south of the equator (see Fig. 2.2). With three sets of these angles obtained with the use of an optical sensor, anglesonly orbit determination methods can be used. The earliest of these is Laplace's method, developed by Pierre-Simon de Laplace (1749-1827) and "remains valid for interplanetary observations"[29]. Another option is that of Gauss' method, which assumes two-body motion to enable conservation of angular momentum and the use of Lagrange coefficients to determine the state vector at the time of the second observation set[8]. Gauss' method can be further improved through an iterative process involving universal Kepler's equations - it is best used for observations of LEO satellites separated by no more than five to ten minutes. The last method, published by Escobal in 1965, is referred to as the Double R-iteration and works well with more widely-spaced observations-observations separated by as much as a full day[29]. Beginning with an initial bounded estimate, the algorithm advances by making additional intermediate guesses from 


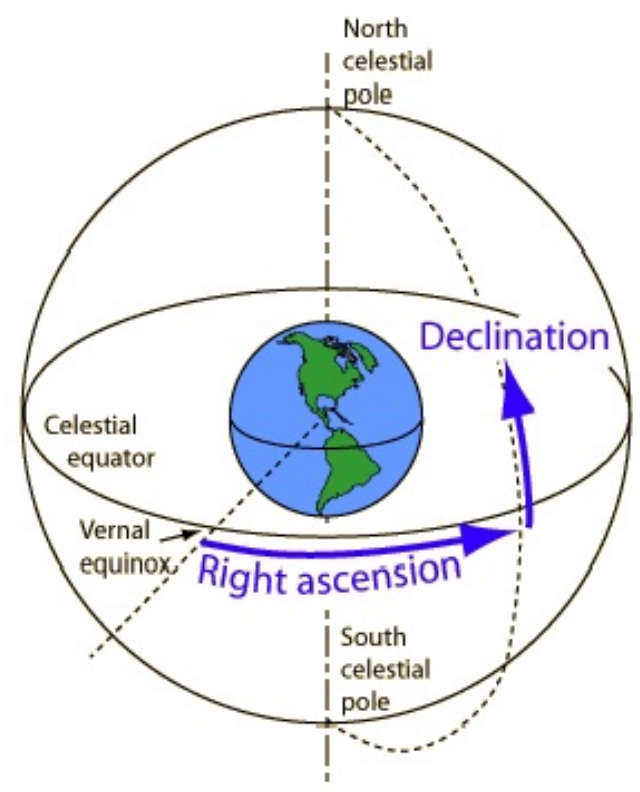

Figure 2.2: Visualization of Right Ascension and Declination within the Celestial Sphere [11]

which differential corrections are made. For a much more in-depth explanation of the ins and outs of orbit determination, see Theory of Orbit Determination by Andrea Milani and Giovanni F. Gronchi[16].

Once position-velocity state vectors have been found through use of the above orbit determination methods, the common orbital elements (COE's) can be calculated. Also known as Keplerian elements, COE's describe the qualities of the orbit through parameters including, but not limited to, the following: semi-major axis, $a$, eccentricity, $e$, inclination, $i$, right ascension of the ascending node, $\Omega$, argument of perigee, $\omega$, and true anomaly, $\nu[29]$. Mean COE's are usually bundled into the TLE's mentioned in the introduction of this paper. These bundles of orbital information - which include additional parameters such as the mean anomaly and B* terms - serve as the initial values for SGP4 propagation. But as one would expect, the accuracy of the TLE-based SGP4 propagation isn't perfect ( $\sim 1 \mathrm{~km}$ at epoch) and error accrues over time[30]. Figures 2.3 and 2.4 show the 

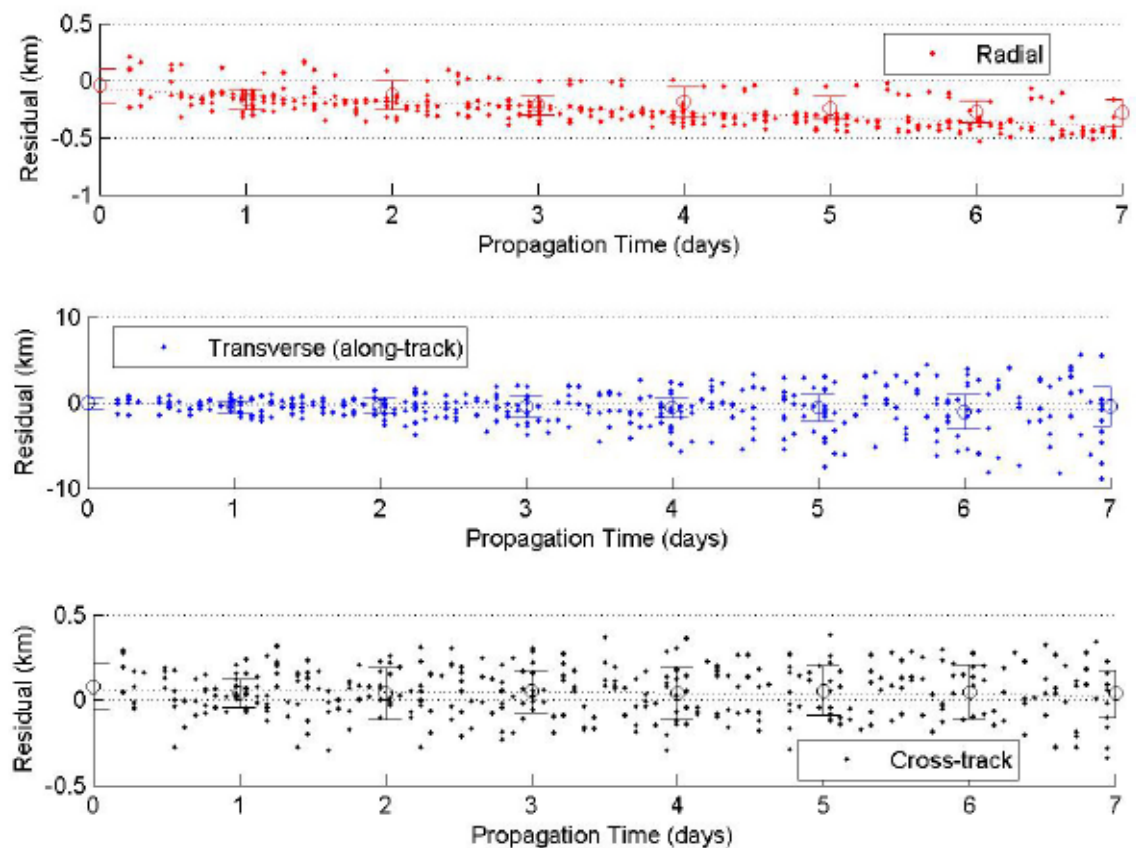

Figure 2.3: Radial, transverse, and cross-track residuals for Stella satellite over 7 days.

temporal error growth of TLE vs. Precision Orbit Ephemerides (POE) for the Stella satellite over the course of seven days[15].

\subsubsection{Satellite Apparent Brightness - Photometry}

Distinct from the problem of astrometry, photometry focuses on analyzing the brightness of a target object. The most simple and straightforward type of photometry is known as differential photometry, which "compares sources sufficiently close together on the sky so that differential first-order extinction can be neglected" [3]. In other words, if two objects such as a satellite and a star are contained within the FOV of a telescope, they are subjected to the same atmospheric effects. Astronomers quantify the brightness of objects with the magnitude scale, a logarithmic scale based upon human vision's nonlinear perception of increases in brightness (all references to magnitude in this paper pertains to apparent 

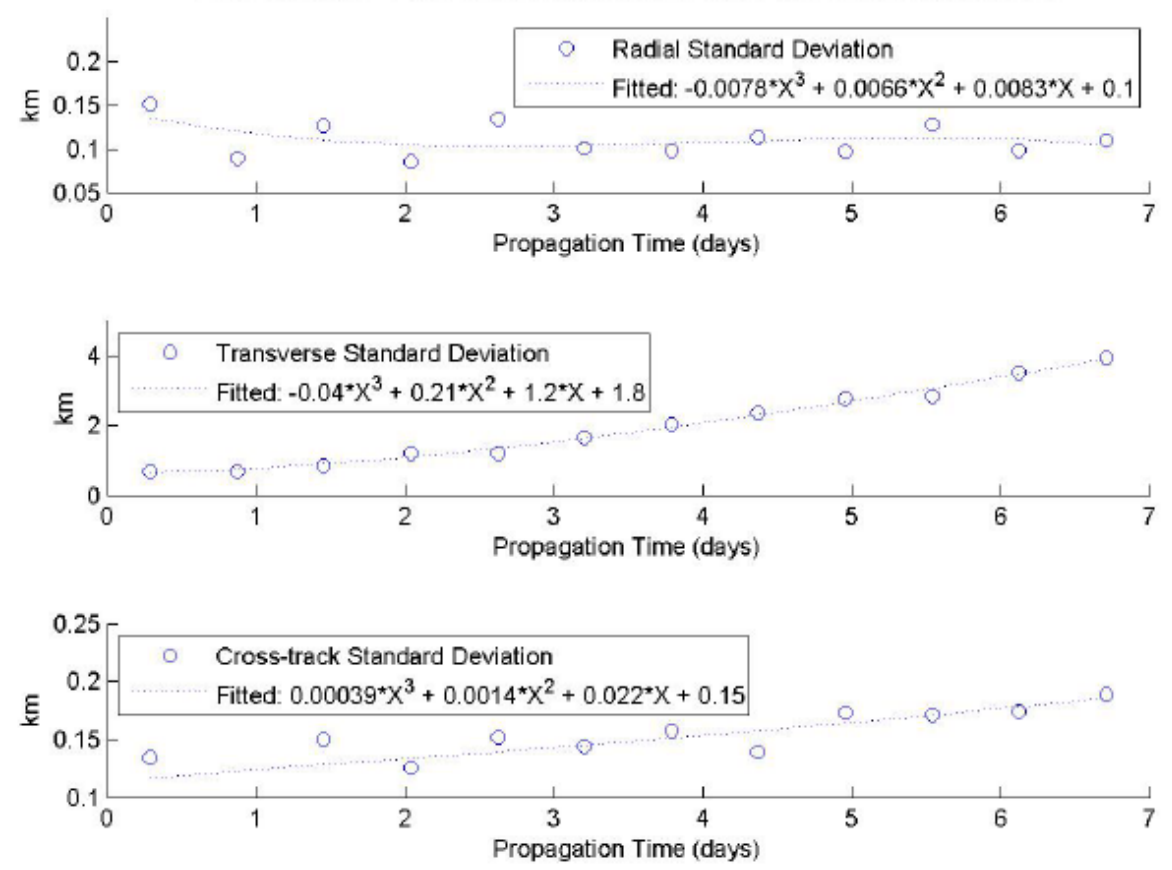

Figure 2.4: Least squares fitted polynomial for standard deviation of radial, transverse, and cross-track residuals for Stella satellite.

magnitude, not to be confused with the standardized scale of absolute magnitude). On the magnitude scale, brighter objects are classified with more negative values - the full moon is approximately - 12.9 magnitude - while dimmer objects receive more positive values - Uranus at its brightest is approximately 5.3 magnitude. The ratio of one object's apparent brightness to another's is converted to magnitudes by the following relationship,

$$
M_{2}-M_{1}=-2.5 \log _{10}\left(\frac{F_{2}}{F_{1}}\right)
$$

where $M$ is magnitude and $F$ is intensity flux. Therefore the +18.2 difference in magnitude between the full moon and bright Uranus is equivalent to a $\sim 2 \times 10^{7}$ reduction in apparent brightness.

Predicting the apparent magnitude for a given satellite is a combination of several factors including but not limited to the following: observatory altitude, observer-to-satellite range, solar phase angle (SPA), elevation angle, design of 


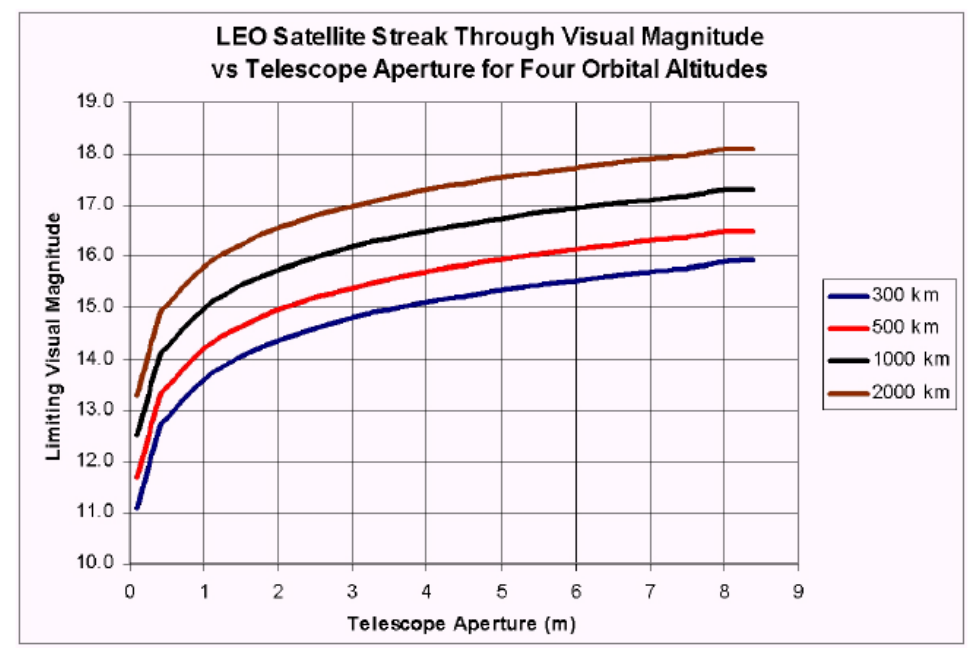

Figure 2.5: Telescope limiting magnitude for a staring system.

telescope, atmospheric seeing conditions, satellite characteristic size, and satellite albedo (reflection coefficient)[1]. An estimate for the expected brightness of target satellite is undeniably important for astronomers, as the difference between the satellite magnitude and the telescope's limiting magnitude dictates the success of the observation. While investigating blind search techniques for micro-satellites in LEO, Ackermann et al. estimated the limiting magnitudes for variable telescope size[1]. These limits are shown in Fig. 2.5-note that this is for a "staring" system (telescope is kept stationary as satellite passes through the FOV), therefore the detection threshold is lower than that for a comparable tracking system. Certain photometry research includes the modeling of debris magnitude, for which a mean albedo value of 0.175 is applied[18]. To estimate the characteristic length, $L_{C}$, of a debris object based upon its apparent magnitude, $M_{a p p}$, a Lambert sphere is assumed and the following equation is used,

$$
L_{C}=10^{\left(M_{\text {Sun }}-M_{a p p}\right) / 5} \frac{R}{\sqrt{A}} \sqrt{\frac{6 \pi}{\sin (S P A)+(\pi-S P A) \cos (S P A)}}
$$

where $M_{\text {Sun }}$ is the magnitude of the Sun, $M_{a p p}$ is the apparent magnitude of the debris object, $\mathrm{R}$ is the range, $\mathrm{A}$ is the albedo, and PA is the solar phase angle. 


\section{Table 2.1: Predicted apparent magnitudes of the CP5 cubesat.}

\begin{tabular}{|c|c|c|c|}
\hline & Mean & Standard Dev. & Ackermann Mean \\
\hline Undeployed Sail & $\mathbf{1 1 . 4 9}$ & $\mathbf{0 . 5}$ & $\sim 10.5$ \\
\hline Deployed Sail & $\mathbf{6 . 9 9}$ & $\mathbf{0 . 5}$ & - \\
\hline
\end{tabular}

Additional research includes obtaining light curves for tumbling rocket bodies[7]. Such information would be pertinent to "plan and develop proximity and docking for potential future Active Debris Removal operations", or to analyze the effects of perturbations upon satellite rotational dynamics.

\subsection{Initial Plan to Observe CP5 Cubesat}

This project began with a specific target in mind: the CP5 cubesat developed by Cal Poly's PolySat laboratory and launched September 13, 2012[22]. It was thought to be an ideal small, LEO satellite for optical observation, particularly due to its deployable sail payload. Normally a 0.1 by $0.1 \mathrm{~m}$ cube $\left(L_{C}\right.$ equal to $0.1414 \mathrm{~m})$, the deployed sail raises the cross-sectional area to $\sim 1 \mathrm{~m}\left(L_{C}\right.$ equal to $1.128 \mathrm{~m}$ ), therefore making it a more viable target. STK and MATLAB were utilized to predict several parameters of CP5 for use in observation preparation, which are shown in Table 2.1 in comparison to predicted values from Ackermann et al[1]. The discrepancy between the values predicted in this report, and those predicted by Ackermann et al., is likely due to differences to some combination of the following factors: observation altitude, albedo, and mean satellite range. Additional predicted values included the mean apparent motion of CP5, 18.6 $\operatorname{arcmin} / \mathrm{s}$, and the maximum apparent motion, $52.7 \mathrm{arcmin} / \mathrm{s}$. But in an unfortunate turn of events, the sail failed to deploy once CP5 was inserted into orbit; due to the failure of the sail deployment, focus was turned toward other satellite targets indefinitely. 
CHAPTER 3

\section{INSTRUMENTATION AND PROCEDURE}

\subsection{Instrumentation}

\subsubsection{Observatory}

Observations were performed at the Cal Poly on-campus dome observatory, shown in Fig. 3.1. While it is located in a convenient location, the observatory suffers from high amounts of light pollution (some effort was made to place boxes over nearby light fixtures) and elevation pointing restrictions. Depending upon the azimuthal direction, the elevation is limited to higher than $20-30^{\circ}$ due to surrounding buildings and trees. Automated tracking is available for the dome, but a delay in response time and slow rotation speed would inhibit attempts at active satellite tracking. The approximate location of the observatory is $35.30^{\circ}$ $\mathrm{N}, 120.66^{\circ} \mathrm{W}$, at an altitude of $105.8 \mathrm{~m}$.

\subsubsection{Telescope}

The main optics of a Meade LX 200 12" telescope was used for this project and can be see in Fig. 3.2. The telescope is an Advanced Coma Free (or SchmidtCassegrain) optical design and and is mounted on a double-tine, fork type mount in the center of the Cal Poly dome observatory. Due to physical constraints on the mount, the telescope is unable to reach within $+/-20^{\circ}$ of North. Table 3.1 lists important specifications of the telescope. At a cost of $\$ 4,499$, this telescope 


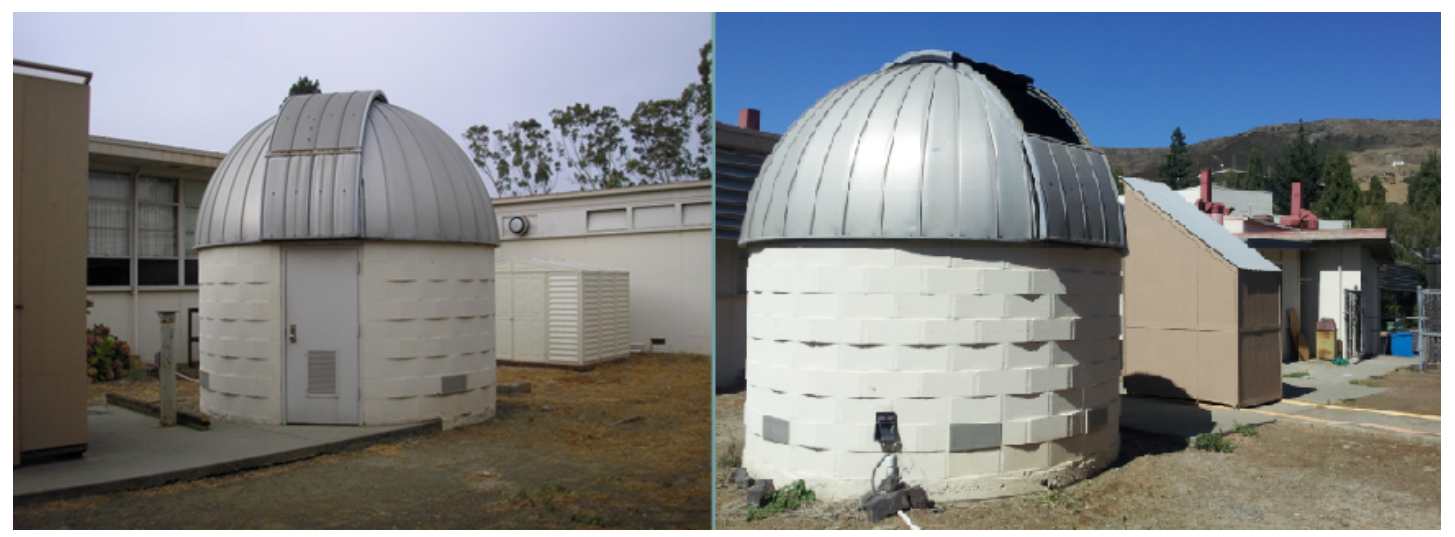

Figure 3.1: Cal Poly Dome Observatory

Table 3.1: Meade LX200 12" Telescope Specifications.

\begin{tabular}{|c|c|}
\hline Specification & Value \\
\hline Aperture & $305 \mathrm{~mm}$ \\
\hline Optical Design & Advanced Coma Free (ACF) \\
\hline Focal Length & $3,048 \mathrm{~mm}$ \\
\hline Focal Ratio & $\mathrm{f} / 10$ \\
\hline Resolving Power & $\mathbf{0 . 3 8}$ arcseconds \\
\hline Eyepiece & $\mathbf{2 6} \mathbf{~ m m}$ Series 4000 Super Plossi \\
\hline Pointing Precision & 1 arcminute \\
\hline
\end{tabular}

is considered to be "amateur-sized", at a price thought to be reasonable for a serious amateur astronomer or undergraduate student.

\subsubsection{Video Camera}

The Lumenera SKYnyx2-0M astrophotography camera was used for recording images produced out of the main optics of the Meade telescope, and is shown in Fig. 3.3. As the camera utilizes a USB interface, it is relatively simple to connect to a computer or laptop for viewing of a live feed or saving of images. Table 3.2 lists the relevant specifications of the camera. This camera is priced at approximately $\$ 1,500$. 


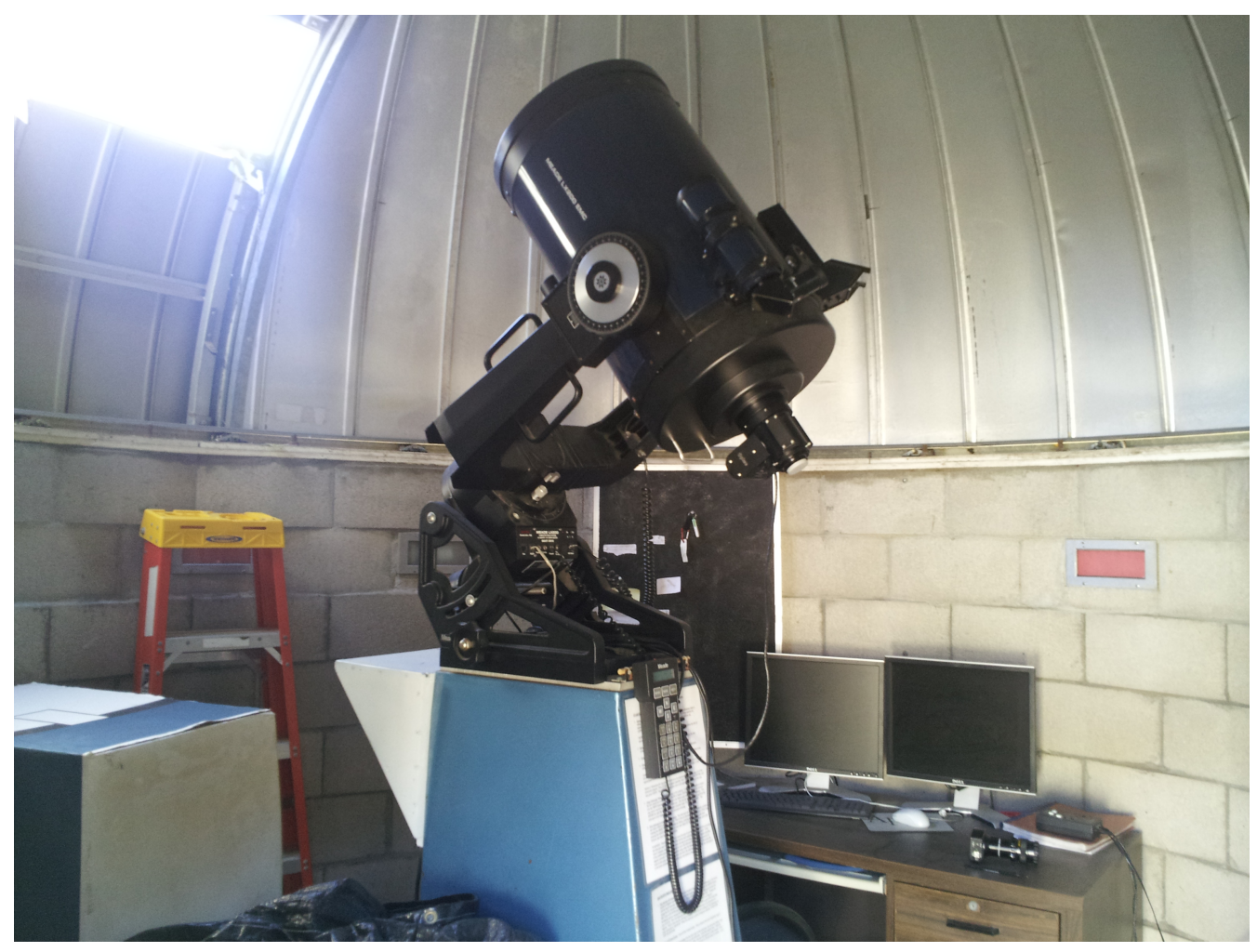

Figure 3.2: Meade LX200 Telescope.

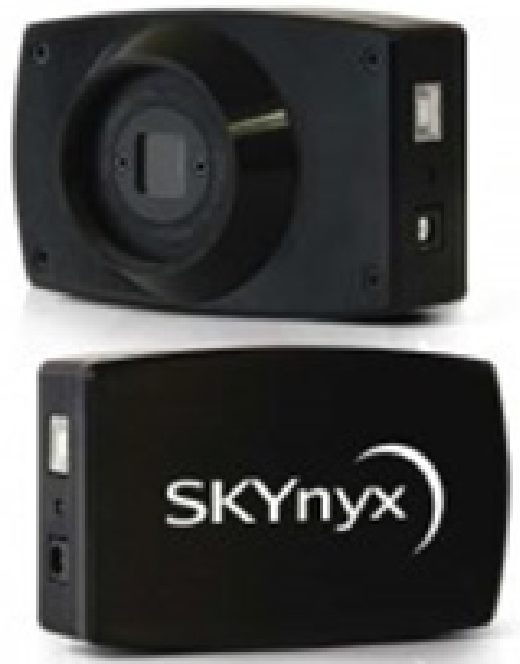

Figure 3.3: Lumenera SKYnyx2-0M Astrophotography Camera. 
Table 3.2: Lumenera SKYnyx2-0M Camera Specifications.

\begin{tabular}{|c|c|}
\hline Image Sensor & Monochrome CCD, $5.8 \times 4.9 \mathrm{~mm}$ array \\
\hline Specification & Value \\
\hline Resolution & $640 \times 480$ pixels \\
\hline Max. Frame Rate & $60 \mathrm{fps}$ \\
\hline Gain Range & 1 to $10 \times$ optimizable \\
\hline Dynamic Range & $>60 \mathrm{~dB}$ \\
\hline Integration Time & $1 / 1000$ to 16 sec. \\
\hline Interface & Standard USB 2.0 high-speed \\
\hline
\end{tabular}

\subsubsection{Focal Reducer}

To increase the FOV of the telescope, a focal reducer was used along with both size and thread adapter barrels. The focal reducer used was a Celestron $\mathrm{f} / 3.3$ - it is pictured in Fig. 3.4. A similar f/6.3 focal reducer is priced at approximately $\$ 130$. Figure 3.5 shows the focal reducer attached to the camera (along with a barrel extension and thread converter) and attached to the back end of the telescope. The decision to incorporate a focal reducer was made when observation failures were incorrectly thought to be occurring due to cross-track error-see Appendix 7.2. However, there are additional advantages gained with the utilization of a focal reducer: a satellite is observed for a longer period of time (reduction in the standard deviation of the observed magnitude value) and the likelihood of the inclusion of secondary companion stars increases. The possible advantages of secondary companion stars are discussed in Section 6.1.

\subsubsection{Software}

Several software applications were involved in the observation process - their uses will be further detailed in the following section. Software Bisque's TheSkyX v10.2 astronomy software was used for computerized control of the telescope in the dome observatory - the Professional edition sells for $\$ 330$. Additional hard- 


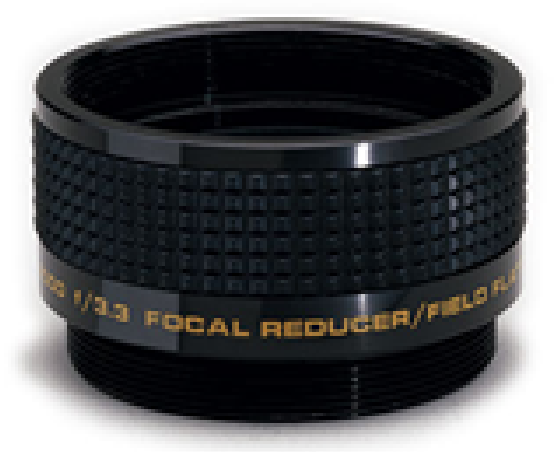

Figure 3.4: Celestron f/3.3 Focal Reducer.

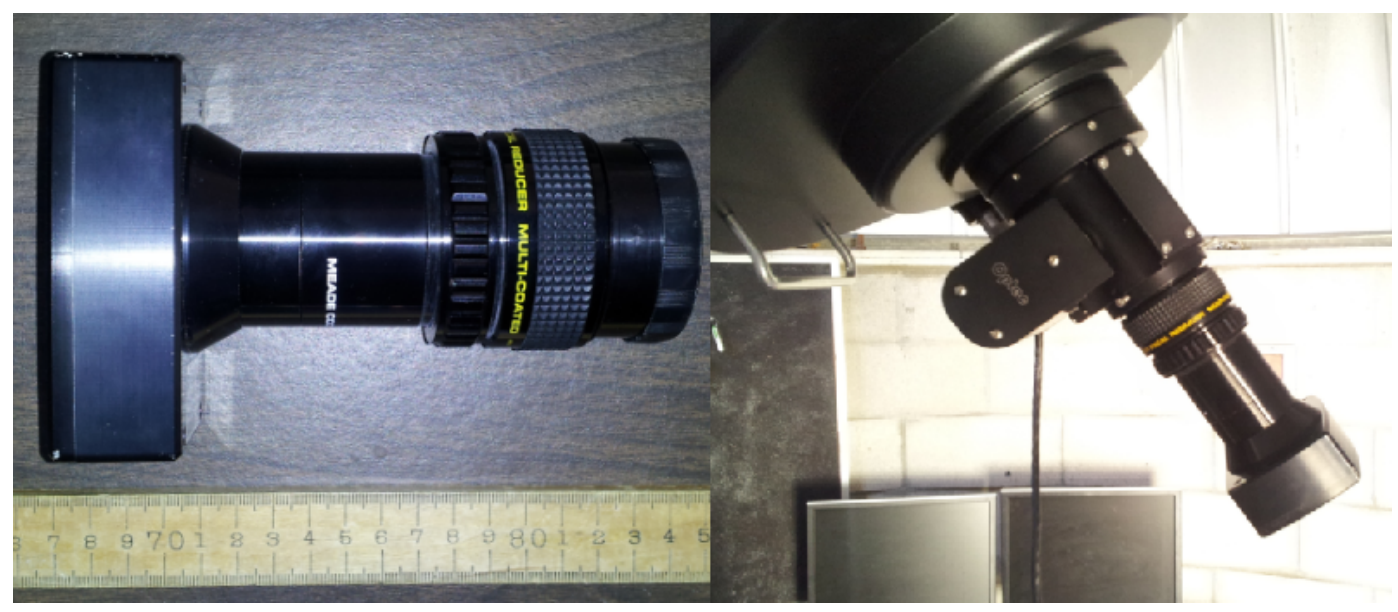

Figure 3.5: Left: full camera setup. Right: camera attached to back end of Meade telescope. 
ware/software involved in the observatory functionality was Technical Innovations' Digital Dome Works v5.2 (DDW) observatory automation system. Such a system allows for a continous, automated tracking of the telescope by the dome. Lucam Recorder v2.7 software was used for control of the Lumenera camera and archiving of recorded images. It is developed by Heiko Wilkens and is available as a free download. For observation preparation and data analysis, MathWorks' MATLAB R2010a and Analytical Graphics Incorporated's STK v10 were used.

\subsection{Procedure}

\subsubsection{Preparation}

On the day of a planned night of observations, preparations normally began two to three hours before astronomical twilight. This process was rather involved, and included the propagation of satellite targets, analysis and selection of those targets, and a generation of observation angles and times at which to observe the targets.

Preparation began with visiting the Space Track website, which provides upto-date TLE data for all documented objects in orbit, and downloading the most recent TLE's for several satellite sets: Globalstar (52 satellites), Visible (214), Weather (57), and Custom (32)[25]. The first three sets are created and main-

tained by the Space Track website, but the Custom list was compiled for the purpose of this project and is shown in Appendix 7.3. Automated download tools are available, such as Celestrak's Space Track TLE Retriever, but were not used for this project[27].

These TLE's were opened by the MATLAB file passPredict.m with the following user-specified inputs: day and time of observation session, duration of 


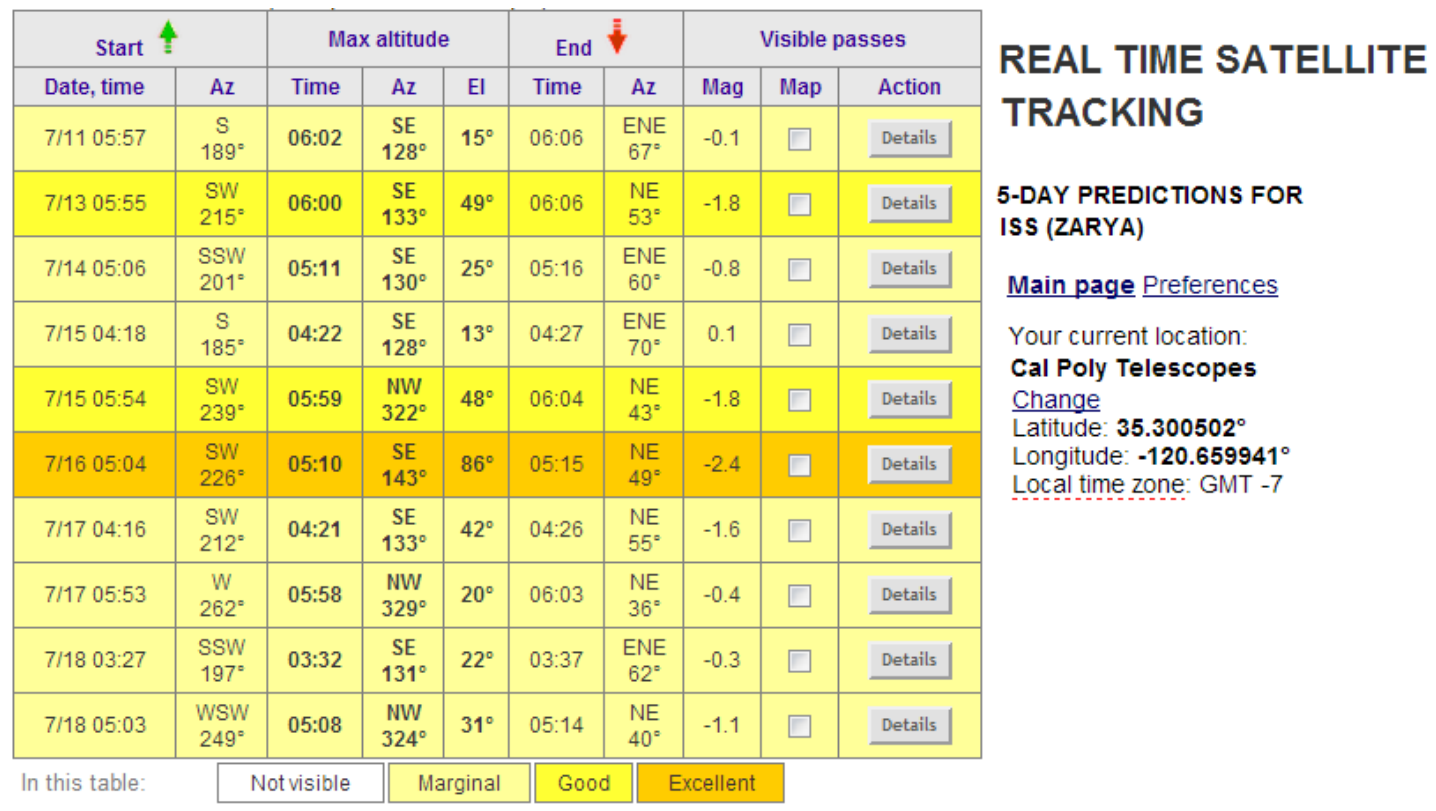

Figure 3.6: Sample Output from N2YO website.

the session, maximum age of the TLE, and minimum elevation attainable by the telescope. Each satellite was propagated forward in its orbit at three minute intervals - satellites found to be both visible (line of sight is possible) and illuminated (at least in partial sunlight) were tagged and the following information was outputted from the code: NORAD ID number, observation time, TLE age, and elevation of observation.

Next, the most attractive targets were selected and their NORAD ID numbers put into the search bar in the real time satellite tracking website n2yo.com[19]. Targets are deemed attractive based upon the length of time they are both visible and illuminated, and their maximum elevation angle; the longer and higher the pass, the better. Maintained by a Ham radio enthusiast (n2yo is their callsign), the website provides valuable information about a satellite's overhead passinformation that is utilized as a means for validation and comparison. Figure 3.6 shows a sample output from the n2yo website. This information is then saved for 
further reference ${ }^{1}$.

Chosen satellites were then inserted into a preexisting STK scenario - if the satellite was already designated within the scenario, only its TLE must be updated via the Space Track option in the TLE Source box within the satellite properties window ${ }^{2}$. SGP4 was then used to propagate through a designated time period, from which an Azimuth-Elevation-Range (AER) report was generated for a given observatory-bounded sensor. The sensor was modeled as Simple Conic type with a $90^{\circ}$ cone half angle. To detect a mid-pass illumination drop-off (a cessation of sunlight partway through the satellite pass), a Lighting Times report was generated ${ }^{3}$. Because LEO satellites are located at such a low altitude, illuminated passes occur for only a few hours after sunset - it is a common occurence that some overhead passes occur at the precise moment that a satellite passes into the Earth's shadow. If such an expiration in illumination was found, the AER report was limited to the times at which the satellite was illuminated. A comma-separated values (csv) version of the AER report was saved at one-second intervals in a Julian date (Jdate) format.

AER reports generated from STK are loaded into MATLAB, at which point the code STK2VIZIER.m was used to convert the azimuth and elevation angles to right ascension and declination angles, which utilizes Algorithms 15 (LSTime.m), 28 (AzElToRaDec.m), 29 (SUN.m), site.m, ecef2eci.m, and razel2rv.m from Vallado[29]. It is worth noting that these RA/Dec angles were then converted from their Current Epoch frame to the more standard J2000 frame (see

\footnotetext{
${ }^{1}$ Because this step is primarily used for prediction validation, with little use in data analysis, it can easily be skipped in any future attempts to replicate this procedure.

${ }^{2}$ Sometime around the release of STK version 10.0.1, the frequency of updates to the AGI server increased from every 2-3 days, to at least once a day. This makes automatic updating of TLE's a more attractive option than manually downloading from Space Track.

${ }^{3}$ This step could potentially be removed if the following two conditions are met: 1) SGP4 propagator used in MATLAB is deemed to be at least as accurate as STK, 2) Propagator interval is significantly reduced from three minutes.
} 


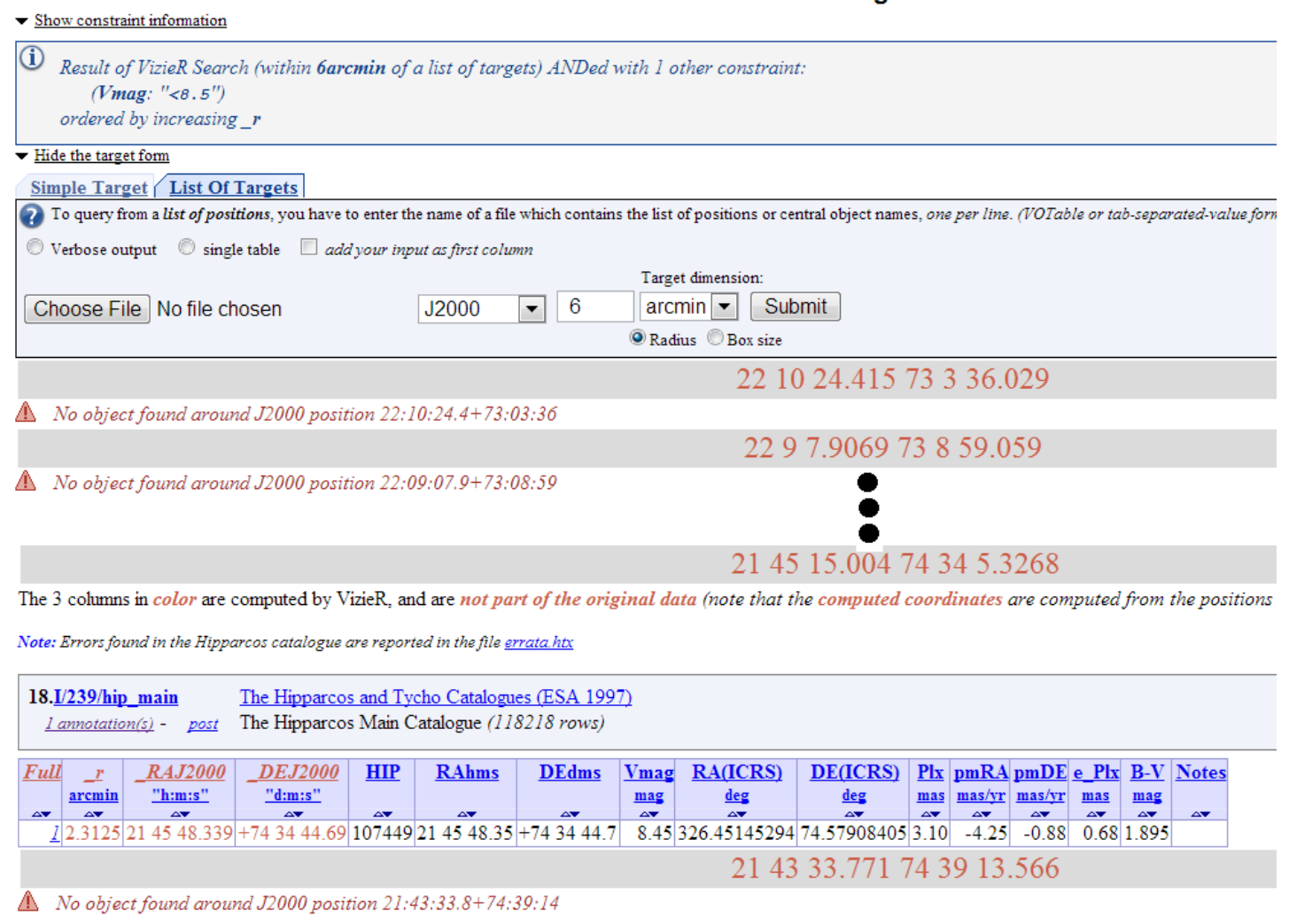

\section{Figure 3.7: Output of VizieR Service for List of Targets Query.}

7.2). These angles were saved to a space-delimited text file, and uploaded to the Strasbourg Astronomical Data Center's (CDS) VizieR service[4]. This service was used to locate stars that qualify as suitable "companion stars" along the projected path of the satellite. At each of the RA/Dec positions, VizieR was assigned to search for stars brighter than 8.5 apparent magnitude (a level visible at the planned exposure time for the camera) and within less than $1 / 2$ the telescope's smaller FOV dimension. The Hipparcos Main Catalogue of 118,218 stars was selected, and the RA/Dec text file was queried as a "List of Targets" with the aforementioned target dimension $(<1 / 2$ FOV). Figure 3.7 displays a few lines of the VizieR output - for a 10 minutes pass, the total number of lines would be 600. Depending upon the intent of the observations, between 1-4 qualifying stars 
were chosen, and the pertinent information was archived for future analysis (star catalog number, star-target separation, and $\mathrm{V}$ magnitude). If orbit determination is the intent of the researcher, then at least three angle sets with a qualifying companion star are required. Because a staring method was used for observing the satellites, and the telescope must be slewed and realigned between each angle set, at least $\sim 2$ minutes was required between each selected set. Between this restriction and those enforced by the pointing limits of the telescope, at times it may be difficult to locate suitable companion stars. There are several methods to combat this problem: 1) reduce the step time to less than one-second intervals, 2) increase exposure time to allow for lower-magnitude companion stars, 3) Query additional star catalogs in VizieR (e.g. Tycho catalog), and 4) increase FOV of the telescope.

Once the angle sets had been selected, the index numbers to the left of the query responses (e.g. "18" in Fig. 3.7) were entered into the latter portion of the STK2VIZIER.m code. This portion of the code returned the following information: time of the observation, azimuth and elevation degree values, satellite range, solar phase angle, and right ascension and declination sexigesimal values. Solar phase angle was calculated with the following method,

$$
\begin{array}{r}
\overrightarrow{r_{A}}=r_{S A T}-r_{S I T E}, \\
\overrightarrow{r_{B}}=r_{S A T}-r_{S U N}, \\
S P A=\arccos \left(\frac{\overrightarrow{r_{A}} \cdot \overrightarrow{r_{B}}}{\left\|\overrightarrow{r_{A}}\right\| \| \overrightarrow{r_{B} \|}}\right)
\end{array}
$$

where $r_{S A T}, r_{S I T E}$, and $r_{S U N}$ are the position vectors of the satellite, observatory, and Sun, respectively. Table 3.3 summarizes the preparation procedure. 
Table 3.3: Information Archived from Preparation.

\begin{tabular}{|c|c|c|c|c|}
\hline Source & MATLAB & N2YO & STK & VizieR \\
\hline Info. & $\begin{array}{c}\text { RA/Dec } \\
\text { SPA } \\
\text { Satellite Name/ID }\end{array}$ & $\begin{array}{l}\text { Satellite Magnitude } \\
\text { Pass start/end times }\end{array}$ & $\begin{array}{c}\text { Illumination Check } \\
\text { Az/Elev } \\
\text { Range }\end{array}$ & $\begin{array}{c}\text { Star Name } \\
\text { Star Magnitude }\end{array}$ \\
\hline & Observation Time & & Observation Jdate & \\
\hline
\end{tabular}

\subsubsection{Observation}

Nights at the Cal Poly Observatory began with activating power to the dome and telescope, and opening DDW and SkyX software. In the attempt to reduce nearby light pollution, boxes were placed over nearby exterior lights and nearby classroom lights were turned off. However this was not always possible, as certain laboratories remained open into the night. Because many observations approached the lower elevation limit of $20^{\circ}$, the lower portion of the dome shutter was raised to the top of the dome - this created an upper elevation limit of $\sim 65^{\circ}$. While a desktop computer was used for dome and telescope control, a separate laptop was used for camera control and image recording. The next step involved attaching the Lumenera camera to the rear of the telescope, and connecting the camera to the laptop via USB. Once a live feed was established, the telescope was commanded to slew to a nearby, bright star (e.g. Sirius). This action served to confirm the dome was tracking the telescope properly, enable synchronization of the telescope with SkyX, and to focus the telescope with the use of an Optec Temperature Compensating Focuser. Excel sheets containing the information gathered during preparation were opened and observations began shortly.

Several minutes before a planned observation, the telescope was commanded to slew to a bright star (>5 magnitude) near to the companion star. Synchronizing with this bright star allows for targeting with the use of the finder scope, and usually guarantees that a small slew $\left(<10-15^{\circ}\right)$ to the companion star will not arrive without the companion star within the FOV of the telescope. Be- 
cause the companion stars were often of a lower magnitude, the finder scope can not be used for synchronization should the telescope lose its pointing accuracyshould this occur, some form of an outward-spiral search pattern must be used with the telescope's hand controls. Considering the time-crunch inherent to the slew-and-stare method, it is much more preferable to use the sync-to-bright-starthen-slew-to-companion-star technique.

Having established that the telescope was accurately pointed at the companion star, a final slew was made to the RA/dec angles set through which the satellite is predicted to pass. Confident that the telescope was now in the right location, the camera remained on standby until 30-60 seconds before the predicted time at which the satellite will pass through the center of the FOV, at which point the recording began. The Lumenera camera was set to an exposure time of $60 \mathrm{~ms}$, maximum gain, and an integration rate of $\sim 17 \mathrm{fps}$, and recorded until 30-60 seconds after the predicted time of satellite passage - Fig. 3.8 shows the active interface of the Lucam Recorder. Images captured by the camera were automatically stored into folders classified by date and time - they were saved in .jpg format and are accompanied by .txt files detailing the stream information (exposure, gain, etc.) and the timestamps for each image. Timestamp accuracy was only as good as the laptop's internal clock, therefore the application Atomic Clock Sync v3.5 was used to synchronize the laptop earlier in the night[6].

Review and analysis of these images, and the archival of the resulting data, will be covered in detail in the following chapter. The entirety of the procedural process is shown step-by-step in Fig. 3.9. 


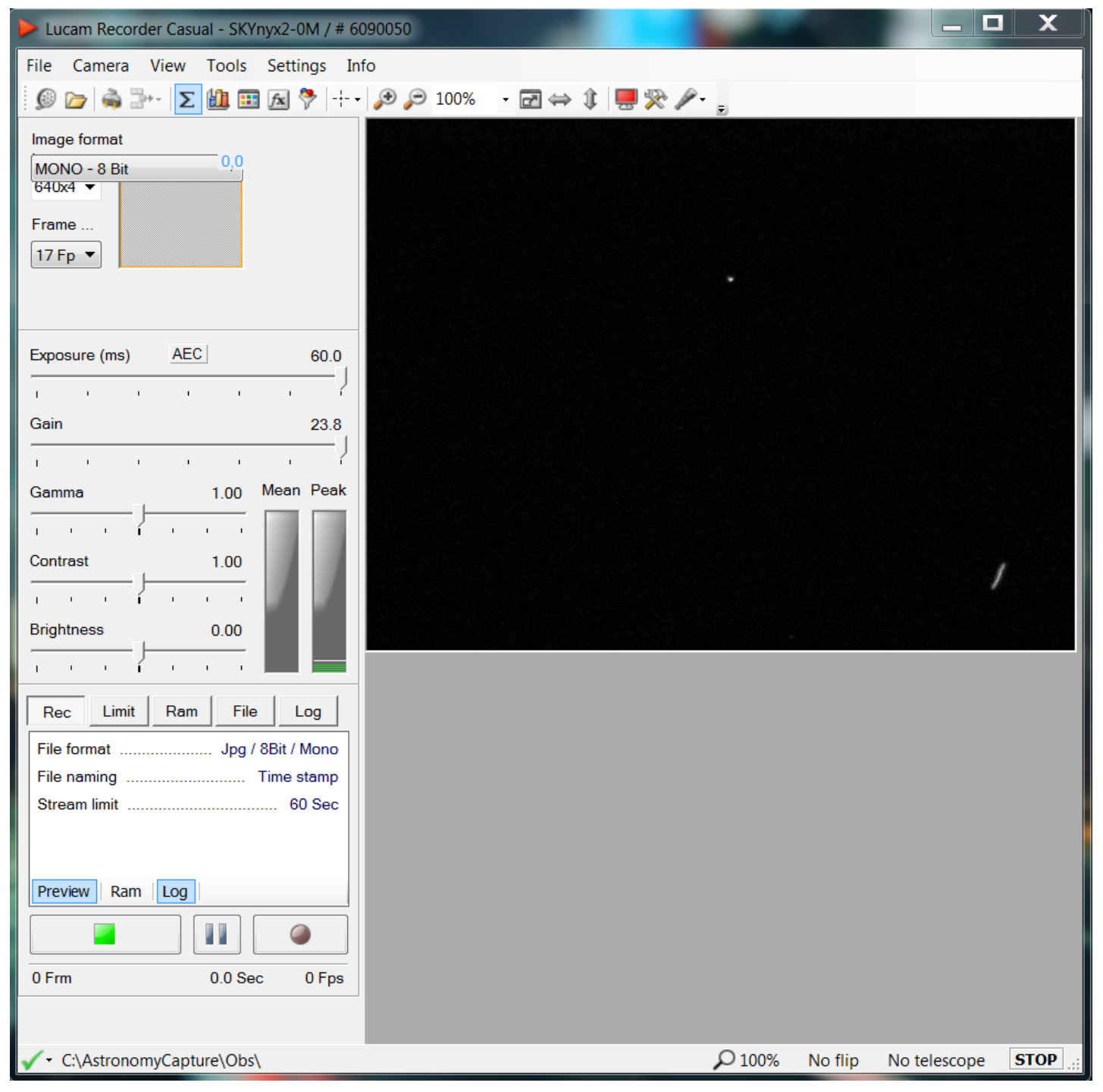

Figure 3.8: Active Interface of Lucam Recorder. 


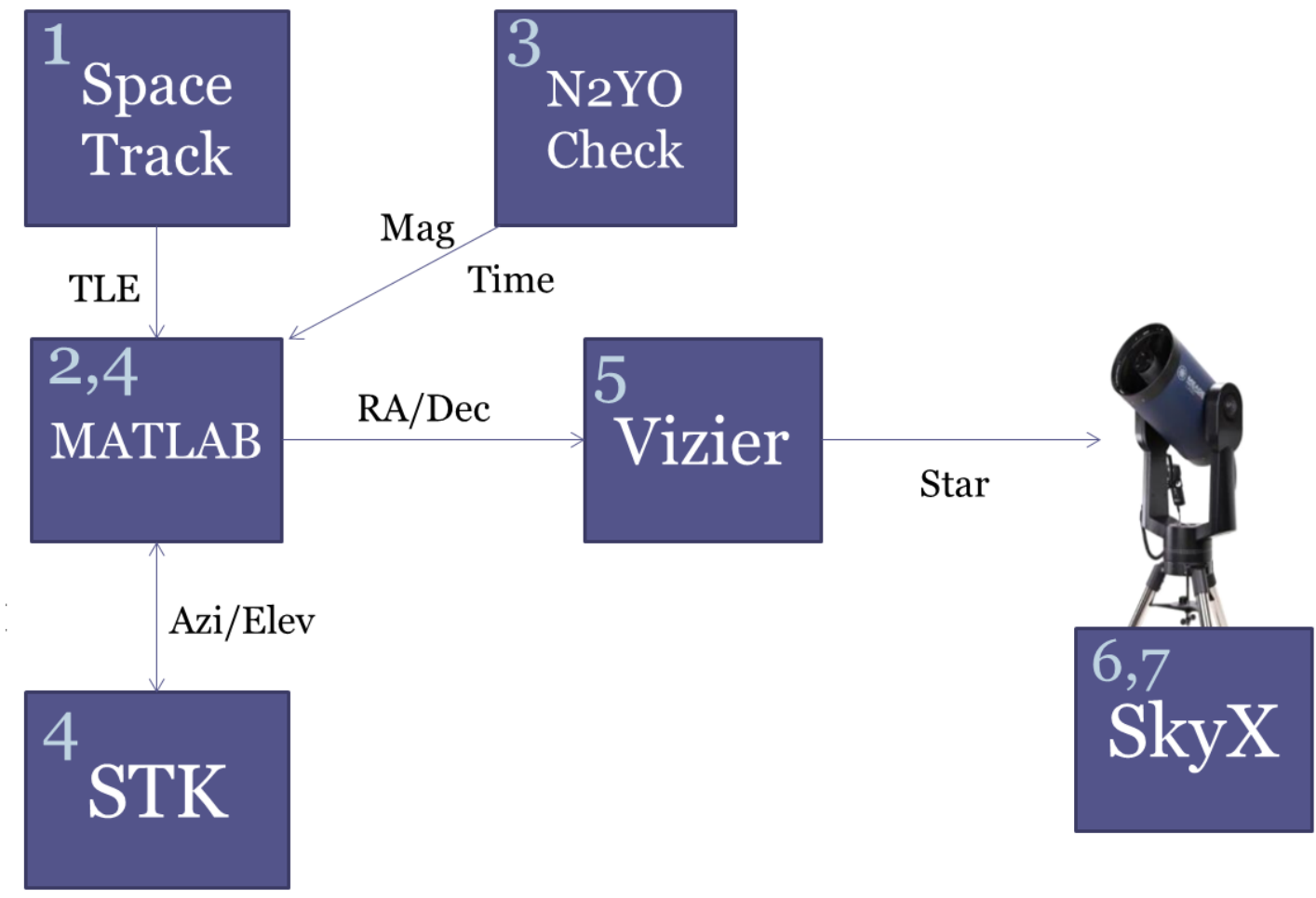

Figure 3.9: Step-by-step Observation Procedure. Includes information sent along each step: satellite Two-Line Element Sets (TLE), satellite apparent magnitude prediction (Mag), time and duration of satellite pass (Time), azimuth and elevation angles (Azi/Elev), right ascension and declination angles (RA/Dec), and companion star details (Star). 


\section{CHAPTER 4}

\section{ANALYSIS}

\subsection{Review of Images}

Images gathered during a night of observation were reviewed by means of a simple, manual "scan" of a set of images. A rather straightforward process, this involved opening a folder of images in Windows Photo Viewer and holding down the right arrow key to view an effective stop-motion animation of the images. At a frame rate of $17 \mathrm{fps}$, a 60 second duration recording would include 1020 images - using this scan method, all images can be viewed in approximately two minutes. With as many as 26 individual observation attempts in a single night, it was found to be both beneficial and efficient to begin scanning images 10 seconds before the predicted pass time, as opposed to 30 seconds. Given that the vast majority of along-track errors were found to be less than a 2-3 seconds, and clock errors of $\sim 5$ seconds or less, the satellites (if visible) would likely be seen within $\sim 30$ seconds. All image review was carried out in a dark room, allowing for easy identification of a moving satellite ${ }^{4}$. If and when the satellite was identified, the images containing the satellite were moved to a separate folder.

\footnotetext{
${ }^{4} \mathrm{~A}$ possible alternative method includes using an application such as Windows Live Movie Maker to both increase the brightness of the images (allowing for easier identification of the satellite) and the speed of the animation.
} 


\subsection{Astrometry}

\subsubsection{Data Reduction}

Conversion from image to data began with identifying the position of the pertinent information within the images. Microsoft Paint was utilized to determine the center $[\mathrm{x}, \mathrm{y}]$ pixel location, pix, of three positions: companion star, first satellite position (after it has fully entered the FOV), and the last satellite position (before exiting FOV), although any similar application that provides cursor information could be used. Because of the finite resolution of the pixels, and the haphazard atmospheric effect of scintillation, an uncertainty of \pm 1 pixel was given to the star positions, and \pm 2 pixels for the satellite positions. The larger uncertainty for satellites is due to the satellites' motion, as a pronounced blurring effect was encountered.

To obtain the necessary data for further analysis, the pixel locations must be converted to right ascension and declination angle sets. This can be accomplished by using the companion star as a reference position, and the direction of satellite's movement as a reference orientation. RA/Dec ephemeris coordinates for cataloged stars can be gathered from CDS's Simbad service - this is an especially helpful tool, as a list of stars can be queried[5]. For establishing the orientation of the FOV, the RA/Dec coordinates saved from the aforementioned STK2VIZIER.m code were used to generate a predicted direction of movement, or slope,

$$
m_{P}=\frac{\delta_{N}-\delta_{T}}{\alpha_{N}-\alpha_{T}}
$$

where the subscripts indicate $T$ for main target position, and $N$ for the next predicted position 1 second later. Replacing the predicted RA/Dec positions in Eq. 4.1 with the pix values for the two satellite positions, the actual FOV slope 
was found,

$$
m_{A}=\frac{p i x_{2 y}-p i x_{1 y}}{p i x_{2 x}-p i x 1_{1 x}} .
$$

To transform from the pixel frame to the RA/Dec frame, the following rotation shown in Fig. 4.1 was made through the correction angle, $\theta$. Any error in the predicted slope - due to an imperfect TLE propagation-was assumed to be negligible at this scale. Although the Meade LX200 telescope utilizes an equatorial mount, which can ensure a constant RA/Dec orientation (given precise camera attachement), the method shown in Fig. 4.1 will work for non-equatorial mounts as well. Now that the pixel frame had been rotated to the correct RA/Dec orientation, the separation vector between the star and satellite position $1, \Delta$, is added to the star's RA/Dec position,

$$
\begin{aligned}
& \theta=\arctan \left(m_{A}\right)-\arctan \left(\frac{m_{P}}{\cos \left(\delta_{\text {mean }}\right)}\right) \\
& {\left[\begin{array}{c}
\delta \\
\alpha
\end{array}\right]_{\text {sat }}=\left[\begin{array}{c}
\delta \\
\alpha
\end{array}\right]_{\text {star }}+\left[\begin{array}{cc}
\cos (\theta) & -\sin (\theta) \\
\sin (\theta) & \cos (\theta)
\end{array}\right]\left[\begin{array}{c}
\Delta_{y} \\
\Delta_{x} / \cos \left(\delta_{\text {mean }}\right)
\end{array}\right] P S}
\end{aligned}
$$

where $P S$ is the plate scale of the image, i.e. the conversion from pixels to arcseconds. The $\cos \left(\delta_{\text {mean }}\right)$ term in Eq. 4.3 was used to scale the right ascension values. Consider the case of longitude and latitude: as latitude increases away from the equator, the angular distance corresponding to a change in longitude decreases, due to the Earth's spherical shape. Therefore, the change in right ascension must be scaled by the mean declination angle at which the observation occurs. The process of determining the RA/Dec positions from the recorded images was handled by the MATLAB code angledet.m. 

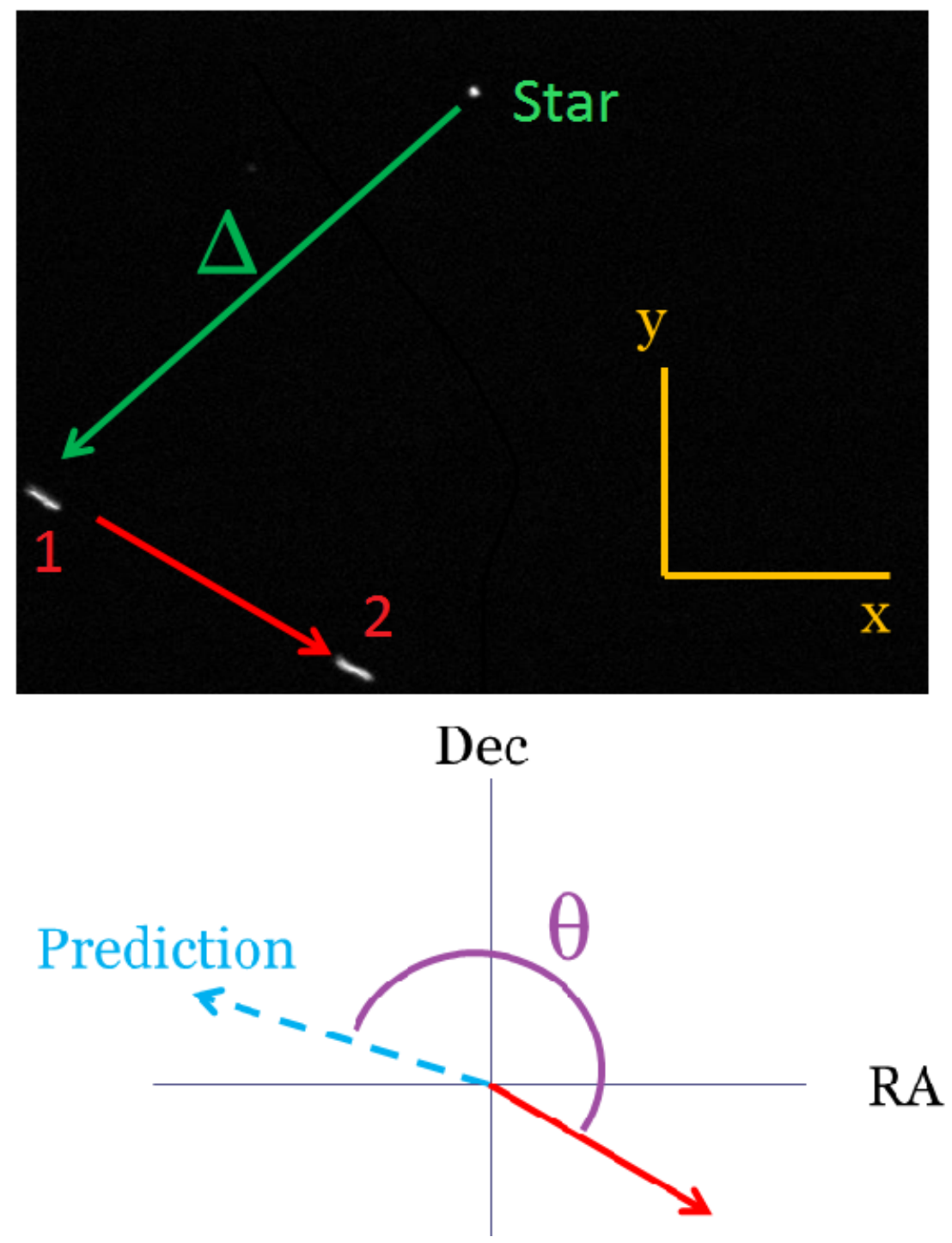

Figure 4.1: Visualization of Pixel-to-RA/Dec Transformation (Pictured top: Satellite movement from position 1 to 2 (red), separation between star and satellite position 1 (green), and frame axes (yellow). Pictured bottom: Predicted direction (blue), actual direction (red), frame rotation (purple), and $\mathrm{RA} /$ Dec axes (black)). 


\subsubsection{Orbit Determination}

Single Angle-Set

As mentioned in Section 2.1.1, the acquisition of at least three RA/Dec anglesets allows for preliminary orbit determination. For all instances in which three angle-sets were obtained, two angles-only methods were used. These included Vallado's rendition of Gauss' Method available online in C++, FORTRAN, Pascal, and MATLAB languages[28]. Scripts based on Vallado's Algorithm 52, ANGLES-DOUBLE-R, and Curtis' rendition of Gauss' Method were also used as a measure of comparison[29][8] . The input required for these methods included the following: line of site vectors, Jdates, and ground site vectors. Line of set vectors, $q$, were calculated from right ascension and declination:

$$
q=\left[\begin{array}{c}
\cos (\delta) \cos (\alpha) \\
\cos (\delta) \sin (\alpha) \\
\sin (\delta)
\end{array}\right]
$$

Jdates were found by converting the saved timestamps for the gathered imagesas a consistent clock error was found in most of the timestamp data, the mean error from a night's observations was removed. Lastly, ground site vectors were found by using site.m to find Earth-Centered, Earth-Fixed position vectors for the Cal Poly Observatory, which were then converted to Earth-Centered Inertial vectors through use of ecef2eci.m. The MATLAB code orbit_determination.m enacted the operations described by this paragraph, and includes a user-specified selection of the desired orbit determination method. Once the position-velocity state vectors were acquired from the output of the orbit determination method, the algorithm RV2COE2.m was used to obtain the COE's for the observed satellite. These parameters provide a comparative measure of success for the

\footnotetext{
${ }^{5}$ Issues were encountered when Vallado's actual Double-R MATLAB code was used.
} 
methods.

Multi Angle-Set

If favorable conditions granted the ability to obtain multiple three-angle-sets for a satellite, either from consecutive passes or on back-to-back nights, more advanced orbit determination methods were used. These advanced methods, an extended Kalman filter (EKF) and a least squares differential corrector (LS), were utilized along with Kepler universal variable propagation to iteratively improve the COE's generated by the initial angles-only orbit determination. For a summarized description of the EKF, see Appendix 7.5. The appendix also details the process noise, covariance, and measurement noise associated with the EKF. Implementation of the EKF and LS was done through the use of orbit_detM.m, a similar code to orbit_determination.m, and extKALMAN2.m and leastsquares.m. A detailed description of nonlinear, least squares differential correction applied to orbit determination can be in Algorithm 66 of Vallado[29].

\subsubsection{Temporal TLE Error Growth}

Considering the imperfect nature of TLE-based propagation, and the inherent difficult in providing an accurate model of orbital perturbations, a measure of temporal TLE error growth is invaluable to a satellite operator. To provide the necessary data for this analysis, observations of a single satellite must be acquired across several days. As a general rule for this project, every satellite successfully captured in a given night was checked for visible passes on the following nights. Should such a visible pass exist - one that does not conflict with a more higher priority observation (such as one involved in orbit determination) - the original TLE used for propagation on the previous night was used to further propagate 
the satellite.

The script pixel_analysis.m also contains the math responsible for this analysis. To determine the distance between the predicted direction of movement, and the actual, observed path, $D$, the following equations were used,

$$
\begin{array}{r}
b_{A}=\delta_{1}-m_{A} \alpha_{1} \\
D=\frac{\left|\delta_{T}-m_{A} \alpha_{T}-b_{A}\right|}{\sqrt{m_{A}^{2}+1}}
\end{array}
$$

where $b$ is the "y-intercept" for the direction of movement. This distance is then converted into the corresponding angular, cross-track error,

$$
C T E=\tan (D) R
$$

which is the observed error tangential to the satellite's direction of movement.

For determining the error in the direction of the satellite's motion, the recorded timestamps were required. The along-track error was found with the following equation:

$$
A T E=t E \sqrt{\frac{\mu}{r_{E}+h}}
$$

where tE is the time error between the predicted time at which the satellite crosses the FOV and the mean time of satellite position 1 and 2-additional values are the Earth's radius, $r_{E}$, Earth's Gravitational parameter, $\mu$, and the satellite altitude, $h$. This equation assumes a circular orbit to determine the orbital velocity, and that the orbital velocity is equal to the transverse velocity seen by the observer. 


\subsection{Photometry}

\subsubsection{Data Reduction}

The method used for analyzing the observed brightness of a satellite was the simple, but well-known, differential photometry approach. Performing this method requires the act of quantifying the level of light associated with both the satellite and the companion star. The astronomical software Image Reduction and Analysis Facility (IRAF) is commonly used for photometric analysis of Flexible Image Transport System (FITS) images. But because images for this project were saved in JPEG format, MATLAB was used to accomplish the photometry. Upon reading and opening images into MATLAB, the command ginput is used to enable the user to save cursor location ( $[\mathrm{x}, \mathrm{y}]$ pixel index) with every click of the mouse. Each pixel within the image carries an intensity "count", I, an integer proportional to the amount of light received by that pixel.

With four clicks, three locales within the image were analyzed: the center of the companion star ( $1^{\text {st }}$ click), two opposite corners of a box surrounding the satellite blur $\left(2^{\text {nd }}\right.$ and $\left.3^{\text {rd }}\right)$, and an empty region of the image $\left(4^{\text {th }}\right)$. Because the atmosphere and seeing conditions were inconsistent, the background noise in each image differs, thereby requiring analysis of a region of the image not host to stars or the satellite. The mean value of an $81 \times 81$ pixel square area surrounding the $4^{\text {th }}$ clicked pixel, was used to estimate the background noise value, $B G$. A $41 x 41$ pixel square area around the $1^{\text {st }}$ clicked pixel, and the square area constrained by the $2^{\text {nd }}$ and $3^{\text {rd }}$ clicks, provided the sum total of pixel intensity values for the 
star, $I_{\text {star }}$, and satellite, $I_{\text {sat }}$, to be used in calculating the magnitude difference:

$$
\begin{array}{r}
F_{\text {star }}=I_{\text {star }}-B G \times N_{\text {star }} \\
F_{\text {sat }}=I_{\text {sat }}-B G \times N_{\text {sat }} \\
M_{\text {sat }}-M_{\text {star }}=-2.5 \log _{10}\left(\frac{F_{\text {sat }}}{F_{\text {star }}}\right)
\end{array}
$$

where $N$ is the number of pixels for the given area (e.g. 1681 for the star area). This process was repeated for each image within a set-depending upon the particular observation, this could number between 1 and $>75$ images. A mean $M_{\text {sat }}-M_{\text {star }}$ value was generated from these images, omitting any outliers seen near the edges of the FOV or when the satellite was in close proximity to a star, along with the standard deviation. Photometric analysis was performed by the code imgPHOT.m and additional details can be found in Appendix 7.4.

\subsubsection{Magnitude Prediction Model}

The MATLAB function appMag.m was developed for this project; the function was purposed with providing a prediction of apparent magnitude for a given satellite pass. Relevant information saved from observation preparation-range, elevation, and SPA — was passed into the function. Beginning with an initial guess for apparent magnitude, Eq. 2.2 was looped until the correct magnitude is found. Satellite albedo is assumed to be 0.175 , whereas the characteristic length of the satellite was found through a more laborious process. While certain sources do provide some amount of satellite dimensions, such as NASA's National Space Science Data Center's Master Catalog, it is often the case that the dimensions are incomplete or unlisted[9]. To provide an estimate of satellite size, the stated mass of a satellite was utilized, along with a mass-volume density relationship. This relationship was determined from a statistical regression of just over two 
dozen satellites with known mass and dimensions,

$$
d=(5.05+0.0353 m a)^{1 / 3}
$$

where $d$ is satellite diameter and $m a$ is mass - it was assumed that the unknown shape of the satellite is a sphere.

Prediction of the satellite magnitude was done for two spacecraft sizes: a small estimate and a large estimate. If multiple dimensions were published for a given satellite, two different initial small and large estimates were provided. For the cases in which either only a single dimension was known, or Eq. 4.9 was used, this single dimension was used for both the small and large estimates. Further separation of the two size estimates was accomplished by the addition of an uncertainty margin - in the interest of modeling satellite drag, researchers have found that a satellite's surface area can vary by at least one order of magnitude[31]. Converting the uncertainty in area, to uncertainty in diameter (assuming circular cross-section), it was found that diameter can vary by $\sim 3.16$. Several test cases were ran, and it was determined that the magnitude predictor was most accurate when $40 \%$ of the margin was given to the small estimate $\left(0.5846 \times d_{\text {small }}\right)$, and $60 \%$ to the large $\left(1.6231 \times d_{\text {large }}\right)$. Size estimation is located within the ObservationResults.m code.

An additional method involving the use of a satellite's ballistic coefficient, found using the TLE B* term, was found to be unsuccessful at accurately predicting the satellite magnitude: predicted values exceeded the large-estimate magnitude prediction by an average magnitude of $\sim 2.9$. It is hypothesized that this inaccuracy is caused by the $\mathrm{B}^{*}$ term's inclusion of additional, time-varying parameters that describe the satellite[31].

Lastly, the effect of atmospheric extinction was applied. As an astronomical object loses elevation in the night sky, its light passes through more atmosphere 
en route to an observer[3]. This increase in path impedance reduces the apparent magnitude of the object by an amount, $k X$, where $k$ is the wavelength-dependent extinction coefficient, and $X$ is air mass and was found by the following expression,

$$
X=\sec (1-e l)\left[1-0.0012\left(\sec ^{2}(1-e l)-1\right)\right]
$$

For a wavelength of $550 \mathrm{~nm}$ (wavelength associated with the V magnitude band), a $\mathrm{k}$ value of 0.17 was used. Both the low and high apparent magnitude predictions, along with the N2YO prediction, which utilizes Michael McCants' method for satellite magnitude estimation, were saved for comparative analysis covered in Chapter 5.

\subsection{Telescope Optimization}

MATLAB's fmincon function, an abbreviation for "find minimum of constrained nonlinear multivariable", is a powerful tool used to optimize a system. Fmincon requires a working model of the system (dynamics of the system driven by input variable(s)), and uses system constraints, variable limitations, and a user-selected optimization algorithm (such as 'interior-point' or 'sequential quadratic programming') to determine the input variables that achieve the best results defined by the system model. For this report, fmincon was used to optimize a telescope purposed for satellite observations. Table 4.1 gives the various elements involved in the optimization scheme, elements detailed in the following paragraph.

In essence, the optimization determines the best telescope size at which to maximize the probability of a successful observation. The optimization process begins with defining the desired qualities of the observation, telescope, and satellite being observed. Once this had been accomplished, the probability of a suc- 
Table 4.1: Details of the Fmincon Telescope Optimizer.

\begin{tabular}{|c|c|c|c|}
\hline Element & Optimizer & Model & Constraints \\
\hline \multirow{2}{*}{ Purpose } & $\begin{array}{c}\text { Initialize fmincon, } \\
\text { Provide visual results }\end{array}$ & $\begin{array}{c}\text { Define system dynamics, } \\
\text { Calculate function value }\end{array}$ & $\begin{array}{c}\text { Enforce limits } \\
\text { on Optimizer }\end{array}$ \\
\hline \multirow{3}{*}{ Inputs } & $\begin{array}{c}\text { Satellite/Telescope qualities: } \\
L_{C}, h, i, \text { TLE age, } \\
n_{T}, \text { minimum } \text { el, } D M D,\end{array}$ & $\begin{array}{c}\text { Optimized variables: } \\
D_{T}, F R, S F R\end{array}$ & $\begin{array}{c}\text { Optimized } \\
\text { variables }\end{array}$ \\
& temporal error growth scheme & & \\
\hline \multirow{2}{*}{ MATLAB } & OptScope & foptScope, PGO & fconScope \\
& & PGOmag & \\
\hline
\end{tabular}

cessful optimization was calculated by determining the probability that the observation will fail due to two causes: A) cross-track error greater than telescope FOV (PGO.m), B) satellite apparent magnitude dimmer than telescope's limiting magnitude (PGOmag.m).

To determine the failure probability due to cross-track error, cause A, the chosen temporal error growth scheme is of utmost importance - either the growth shown in Fig. 2.4, or the results of Section 5.2 were used. Given the age of the TLE used for orbit propagation, an estimate of CTE was calculated. As for the FOV of the telescope, the following equation was used,

$$
F O V=\frac{0.833 n_{T}\left(\frac{12}{D_{T}}\right)}{S F R / 10}
$$

where $n_{T}$ is the number of telescopes should an optical fence be used, $D_{T}$ is the telescope diameter in inches, and SFR is the telescope's focal ratio. Assuming that the the orbit propagation error follows a normal distribution, the probability of a successful observation was found in the following manner,

$$
\begin{gathered}
x=\frac{F O V}{C T E} \\
\text { fProbability }(y)=\frac{\exp \left(\frac{-y^{2}}{2}\right)}{\sqrt{(2 \pi)}} \\
\text { Probability }=\int_{-x}^{x} \text { fProbability }(y) d y
\end{gathered}
$$

where $f$ Probability is the probability density function. 
Considering cause B for an observational error, it is first necessary to determine the effective magnitude limit of the telescope used for observations. The telescope limiting magnitude, TLM, is a function of the telescope's optical geometry, the camera exposure time, and the seeing conditions for a given night. For optimization purposes, however, seeing conditions were not considered. Unlike the user-specified exposure time that presides elsewhere in this project, the exposure time used for optimization is itself optimized - exposure time is set to $1 / 10^{\text {th }}$ the time the satellite takes to pass through the FOV. To simplify this calculation, an assumption of a circular orbit was used for calculating satellite range and velocity, $v$. The probability of a successful observation, in regards to the telescope limiting magnitude, was found in the following manner,

$$
\begin{gathered}
A M=\cot \left(\frac{v}{R}\right) \\
E T=\frac{F O V}{10 A M} \\
T L M=10+1.25 \log (E T)-D M D+2.1715 \log \left(D_{T} \sqrt{\frac{S F R \times F R}{10}}\right) \\
\text { fProbability }(y)=\frac{\exp \left(-\frac{1}{2}\left(\frac{y-M_{s a t}}{1.0993}\right)^{2}\right)}{1.0993 \sqrt{(2 \pi)}} \\
\text { Probability }=\int_{-20}^{T L M} \text { fProbability }(y) d y
\end{gathered}
$$

where $A M$ is the apparent motion of the satellite, ET is exposure time, and DMD is the desired magnitude difference between the theoretical telescope limiting magnitude and the limiting magnitude used for optimization. DMD was used as a means to apply margin to the optimization, and was set to a value of 1 magnitude. A mean magnitude prediction error of 0.95 and an error standard deviation of 0.75 , found from the results of the photometry analysis, give a probability density function with a standard deviation of 1.07. Both Eq. 4.12a and 4.13a utilized 
Table 4.2: Fmincon Optimizer Constraints.

\begin{tabular}{|c|c|c|c|}
\hline Constraint & Minimum & Maximum & Initial Value \\
\hline Telescope Diameter & $3 \mathrm{in}$. & 20 in. & 12 in. \\
\hline Focal Reduction & 0.2 & 1 & 0.6 \\
\hline Telescope Length & 14 in. & 34.5 in. & 26 in. \\
\hline Focal Ratio & 5 & 20 & 10 \\
\hline
\end{tabular}

relationships determined analytically with Sky \& Telescope's Scope Calculator utility[2].

The product of these two probability values was the value-of-interest for the optimizer-fmincon attempts to minimize the value with respect to the constraints on the system. These constraints are listed in Table 4.2, as well as the initial guessed values for the optimized variables. The variable limits were chosen based on the common dimensions seen in amateur-sized telescopes priced under $\sim \$ 10,000$. Various test cases will be shown in the following chapter. 


\section{CHAPTER 5}

\section{RESULTS AND DISCUSSION}

From February to May, observations were made on 29 separate nights, providing a total of 186 individual attempts at imaging a satellite. This does not include at least a dozen planned nights of operation, upon which either weather or a scheduling conflict (observatory was shared between several research projects) prevented any observations. Of the individual attempts conducted, 96 were successful-22 different satellites were captured and are shown through STK in Figure 5.1. Due to their large number and relatively high altitudes, Loral's Globalstar spacecraft made for ideal and consistent targets; Globalstar spacecraft accounted for 17 of the satellites captured.

Whereas 96 successful captures could be used for photometric analysis, only a fraction were suitable for astrometry. The requisite observational data for orbit determination consists of three consecutive angle sets, hereto known as "triocaptures". Over the course of this study, 7 distinct, single trio-captures were acquired. To enable supplementary use of EKF or LS, at least two consecutive trio-captures are required - three double trio-captures were obtained. As for analysis of temporal TLE error, observations of satellites obtained on consecutive days are needed -9 satellites were captured multiple times during a 2-6 day period, pertaining to a total of 42 observations. Several image sets can be seen in Appendix 7.1. 

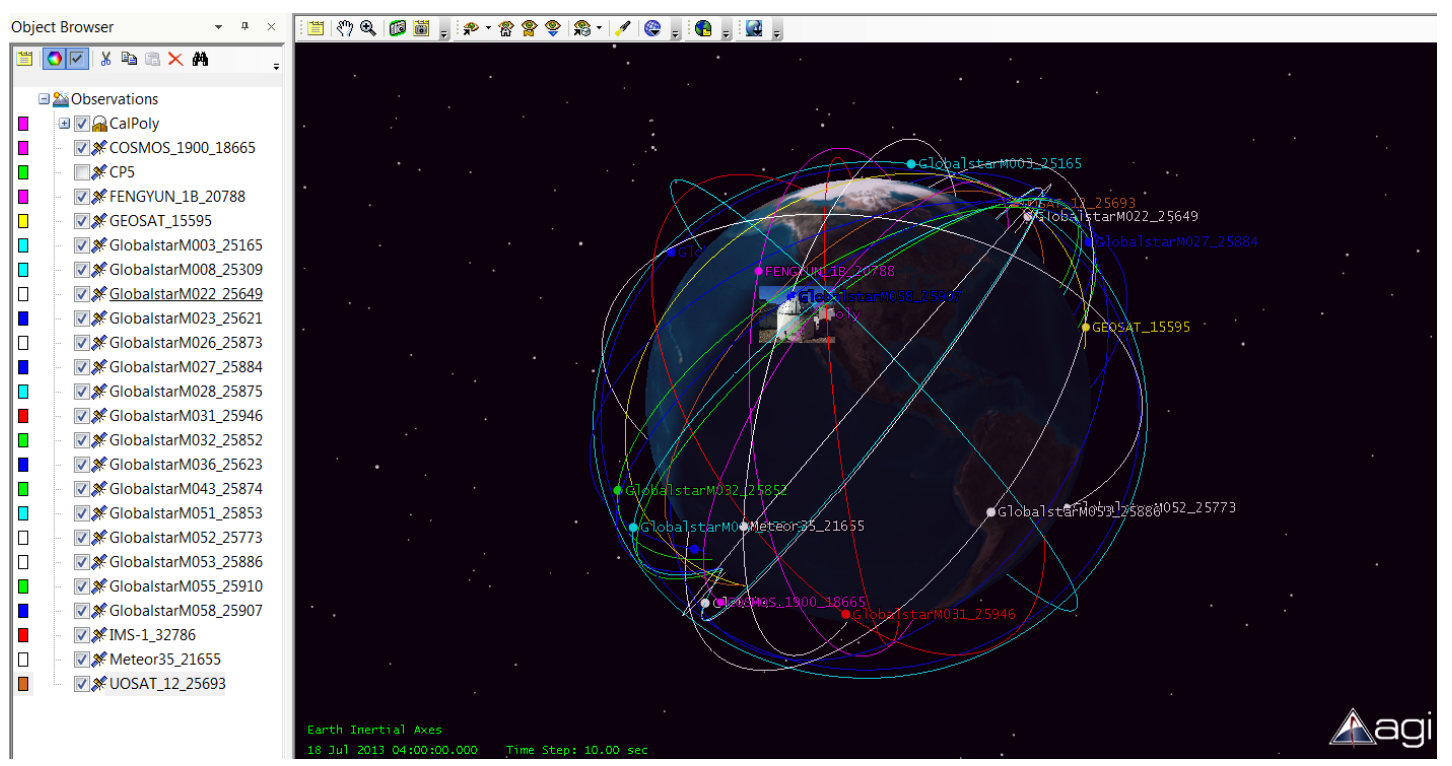

\section{Figure 5.1: Successfully Observed Satellites listed within STK Sce- nario.}

\subsection{Orbit Determination}

To present the results of the various orbit determination methods, direct comparisons are made between the determined COE's and the actual, true COE's. Truth data is gathered from the original TLE's used for orbit propagation, whereupon it is converted from mean to osculating elements by way of Kwok's method (Vallado 9.9.1), handled by the code mean2osc.m[29]. Table 5.1 presents the results of three of the single trio-captures - results of the remaining captures can be found in Table 7.4 of Appendix 7.1. The uncertainty values for the double-r method, shown in Table 5.1, arise from the pixel-selection method-to analyze this error, a simple Monte-Carlo method was used, the details of which are given in Appendix 7.6. Uncertainty values were only given for the double-r method, as the differences in results of the various orbit determination methods (differences in both the COE's and COE uncertainties) were found to be insignificant. For this same reason, only the double-r method was used for the testing of the EKF 
Table 5.1: Single Pass Orbit Determination Results.

\begin{tabular}{|c|c|c|c|c|c|c|c|}
\hline Satellite & Source & $\operatorname{Epoch}(\mathrm{UT})$ & $\mathrm{A}(\mathrm{km})$ & $e$ & $i\left({ }^{\circ}\right)$ & $\Omega\left({ }^{\circ}\right)$ & $\omega\left(^{\circ}\right)$ \\
\hline \multirow{4}{*}{$\begin{array}{c}\text { Globalstar } \\
\text { M003 }\end{array}$} & TLE & $3 / 25 \quad 18: 53$ & 8001.4 & $9.275 \mathrm{E}-4$ & 52.02 & 299.16 & 127.39 \\
\hline & Vallado Gauss & \multirow{3}{*}{ 3/25 3:15 } & 8666.3 & $6.646 \mathrm{E}-2$ & 51.69 & 300.26 & 147.61 \\
\hline & Curtis Gauss & & 8618.3 & $6.548 \mathrm{E}-2$ & 51.85 & 300.45 & 146.89 \\
\hline & double-r & & $\begin{array}{c}8618.3 \\
\pm 49.2\end{array}$ & $\begin{array}{c}6.547 \mathrm{E}-2 \\
\pm 0.492 \mathrm{E}-2\end{array}$ & $\begin{array}{l}51.85 \\
\pm 0.02\end{array}$ & $\begin{array}{c}300.45 \\
\pm 9.5\end{array}$ & $\begin{array}{c}146.89 \\
\pm 0.86\end{array}$ \\
\hline \multirow{4}{*}{$\begin{array}{c}\text { Globalstar } \\
\text { M031 }\end{array}$} & TLE & 4/14 3:56 & 7856.9 & $3.086 \mathrm{E}-4$ & 52.00 & 329.54 & 145.10 \\
\hline & Vallado Gauss & \multirow{3}{*}{$4 / 143: 56$} & 7050.9 & $1.058 \mathrm{E}-1$ & 52.29 & 329.95 & 324.56 \\
\hline & Curtis Gauss & & 7044.6 & $1.044 \mathrm{E}-1$ & 52.39 & 330.06 & 324.67 \\
\hline & double-r & & $\begin{array}{c}7044.6 \\
\pm 4.6\end{array}$ & $\begin{array}{c}1.044 \mathrm{E}-1 \\
\pm 0.007 \mathrm{E}-1\end{array}$ & $\begin{array}{l}\mathbf{5 2 . 3 9} \\
\pm 0.01\end{array}$ & $\begin{array}{r}330.06 \\
\pm 10.55\end{array}$ & $\begin{array}{c}324.67 \\
\pm 0.09\end{array}$ \\
\hline \multirow{4}{*}{$\begin{array}{c}\text { Globalstar } \\
\text { M036 }\end{array}$} & TLE & 4/20 11:06 & 8121.7 & $1.379 \mathrm{E}-3$ & 52.00 & 64.18 & 180.56 \\
\hline & Vallado Gauss & \multirow{3}{*}{$4 / 206: 10$} & 8998.4 & $8.714 \mathrm{E}-2$ & 52.51 & 62.19 & 112.37 \\
\hline & Curtis Gauss & & 8924.7 & $8.414 \mathrm{E}-2$ & 52.35 & 62.06 & 112.95 \\
\hline & double-r & & $\begin{array}{c}8924.7 \\
\pm 10.2\end{array}$ & $\begin{array}{c}8.414 \mathrm{E}-2 \\
\pm 0.097 \mathrm{E}-2\end{array}$ & $\begin{array}{c}52.35 \\
\pm 0.004\end{array}$ & $\begin{array}{c}62.06 \\
\pm 7.8\end{array}$ & $\begin{array}{c}112.95 \\
\pm 0.07\end{array}$ \\
\hline
\end{tabular}

and LS.

In an attempt to gain insight into the source of error responsible for the COE error, the determined and actual state vectors are compared in Table 5.2. Coefficient of determination, $\mathrm{R}^{2}$, values were calculated to reveal any correlation between the state error and the uncertainty in the states (proportional to the states) - large correlation could be an indication that the Monte Carlo error analysis, while correct regarding the sources of error, applied insufficient levels of measurement error. The varied $\mathrm{R}^{2}$ results indicate that it is likely that additional, unknown sources of measurement or process error are responsible for the state errors. Equations used to derived the COE's from the state vectors are shown in Appendix 7.4, in RV2COE2.m, and may be helpful in determining the cause of error in the orbit determination results.

Also apparent in Table 5.2, is an imbalance between the errors in velocity vectors and the errors in the radius vectors - velocity vectors routinely contained larger relative error. For nine cases of single-pass orbit determination (including the first three used in the multi-arc orbit determination), the determined $\mathrm{COE}$ and state vector errors were tested for correlation with the use of Minitab. The resulting Pearson correlation coefficients, and their respective p-values, are given 
Table 5.2: Single Pass Orbit Determination Results cont.

\begin{tabular}{|c|c|c|c|c|c|c|c|}
\hline Satellite & Source & $\mathbf{r}_{x}(\mathbf{k m})$ & $\mathbf{r}_{y}(\mathbf{k m})$ & $\mathbf{r}_{z}(\mathbf{k m})$ & $\mathbf{v}_{x}(\mathbf{k m} / \mathbf{s})$ & $\mathbf{v}_{y}(\mathrm{~km} / \mathrm{s})$ & $\mathbf{v}_{z}(\mathrm{~km} / \mathrm{s})$ \\
\hline \multirow{5}{*}{$\begin{array}{c}\text { Globalstar } \\
\text { M003 }\end{array}$} & TLE & -860.85 & 7049.60 & 3696.95 & -5.1443 & 1.7407 & -4.5052 \\
\hline & \multirow{2}{*}{ double-r } & -813.03 & 7104.22 & 3697.34 & -5.2637 & 1.8140 & -4.6311 \\
\hline & & \pm 1.36 & \pm 4.77 & \pm 1.63 & \pm 0.0081 & $\pm \mathbf{0 . 0 0 9 8}$ & \pm 0.0111 \\
\hline & Error (\%) & 5.56 & 0.77 & 0.01 & 2.32 & 4.21 & 2.79 \\
\hline & $\mathbf{R}^{2}$ & \multicolumn{6}{|c|}{0.36} \\
\hline \multirow{5}{*}{$\begin{array}{c}\text { Globalstar } \\
\text { M031 }\end{array}$} & TLE & -4179.73 & 5643.08 & 3511.53 & -5.3220 & -1.0575 & -4.6223 \\
\hline & double-r & -4160.57 & 5553.84 & 3533.78 & -5.0887 & -1.0255 & -4.4279 \\
\hline & Govite-1 & \pm 0.45 & $\pm \mathbf{0 . 7 9}$ & $\pm \mathbf{0 . 1 8}$ & $\pm \mathbf{0 . 0 0 1 7}$ & $\pm \mathbf{0 . 0 0 1 4}$ & $\pm \mathbf{0 . 0 0 1 6}$ \\
\hline & Error (\%) & 0.46 & 1.58 & 0.63 & 4.39 & 3.03 & 4.20 \\
\hline & $\mathbf{R}^{2}$ & \multicolumn{6}{|c|}{0.17} \\
\hline \multirow{4}{*}{$\begin{array}{c}\text { Globalstar } \\
\text { M036 }\end{array}$} & TLE & -5648.03 & -1100.79 & 5716.33 & -1.2410 & -6.4438 & -2.4736 \\
\hline & double-r & $\begin{array}{c}\mathbf{- 5 6 5 2 . 9 0} \\
\pm 0.15\end{array}$ & $\begin{array}{c}-1103.76 \\
\pm 0.74\end{array}$ & $\begin{array}{c}\mathbf{5 7 6 4 . 1 7} \\
\pm \mathbf{0 . 8 2}\end{array}$ & $\begin{array}{l}\mathbf{- 1 . 3 0 6 1} \\
\pm \mathbf{0 . 0 0 1 0}\end{array}$ & $\begin{array}{l}\mathbf{- 6 . 5 9 2 6} \\
\pm 0.0027\end{array}$ & $\begin{array}{l}\mathbf{- 2 . 5 1 1 1} \\
\pm \mathbf{0 . 0 0 1 0}\end{array}$ \\
\hline & Error (\%) & 0.09 & 0.27 & 0.84 & 5.25 & 2.31 & 1.51 \\
\hline & $\mathbf{R}^{2}$ & \multicolumn{6}{|c|}{0.40} \\
\hline
\end{tabular}

for correlations below a significance level of 0.15 . Pearson correlation coefficients are located on a scale from -1 to 1: -1 indicates perfect inverse correlation, 1 indicates directly-proportional correlation, and 0 indicates no correlation. A p-value of 0.1 indicates that there is a $10 \%$ chance that the correlation is coincidental. Because the number of data points are few, the results shown in Table 5.3 are not definitive, and should not be considered representative of all angles-only observations. Because it is not immediately clear how state vector errors translate into measurement errors, or explain the shortfalls of the observation process, two observational parameters were also tested for correlation: arc distance, $\Delta \mathrm{t}$ (time duration covered by a trio-capture), and range coverage, $\Delta \mathrm{R}$ (difference in minimum and maximum range within a trio-capture). As correlation likely exists between the $i, a, e$, and $\omega$ errors, and error is correlated between many of the state vector dimensions, it may be the case that additional correlation between the COE's and states exist, but are not reflected by the data. The only COEstate error correlations at a significance lower than 0.15 are those between $\mathrm{r}_{y}$ and $e$, and $\mathrm{r}_{y}$ and $i$. However, if correlations can be treated as syllogistic (if A implies $\mathrm{B}$ and $\mathrm{B}$ implies $\mathrm{C}$, then $\mathrm{A}$ implies $\mathrm{C}$ ), then it can be hypothesized that increases in arc distance and range coverage effectively decrease the errors in $i, a, e$, and 
Table 5.3: Correlation between Determined COE and State Vector Errors, and Observational Parameters (Obs.).

\begin{tabular}{|c|c|c|c|c|}
\hline Type & Element 1 & Element 2 & Correlation & p-value \\
\hline \multirow{5}{*}{ COE-COE } & $i$ & $a$ & 0.91 & 0.001 \\
\hline & $i$ & $\omega$ & 0.62 & 0.072 \\
\hline & $i$ & $e$ & 0.62 & 0.078 \\
\hline & $e$ & $a$ & 0.55 & 0.122 \\
\hline & $e$ & $\omega$ & 0.53 & 0.141 \\
\hline \multirow{5}{*}{ State-State } & $\mathbf{r}_{z}$ & $\mathbf{v}_{x}$ & 0.91 & 0.001 \\
\hline & $\mathbf{r}_{y}$ & $\mathbf{r}_{z}$ & 0.77 & 0.016 \\
\hline & $\mathbf{v}_{x}$ & $\mathbf{v}_{z}$ & 0.75 & 0.02 \\
\hline & $\mathbf{r}_{y}$ & $\mathbf{v}_{x}$ & 0.58 & 0.101 \\
\hline & $\mathbf{r}_{z}$ & $\mathbf{v}_{z}$ & 0.58 & 0.101 \\
\hline \multirow{2}{*}{ COE-State } & $e$ & $\mathbf{r}_{y}$ & 0.68 & 0.043 \\
\hline & $i$ & $\mathbf{r}_{y}$ & 0.58 & 0.1 \\
\hline \multirow{5}{*}{ State-Obs. } & $\Delta \mathbf{R}$ & $\mathbf{v}_{z}$ & -0.81 & 0.008 \\
\hline & $\Delta \mathbf{t}$ & $\mathbf{r}_{z}$ & -0.58 & 0.1 \\
\hline & $\Delta \mathbf{R}$ & $\mathbf{v}_{x}$ & -0.58 & 0.105 \\
\hline & $\Delta \mathbf{t}$ & $\mathbf{v}_{z}$ & -0.55 & 0.124 \\
\hline & $\Delta \mathrm{t}$ & $\mathbf{v}_{x}$ & -0.54 & 0.131 \\
\hline
\end{tabular}

$\omega$.

Additional observational data could provide clearer relationships between the observational parameters and determined COE error, and could in turn, provide beneficial modifications to satellite observation techniques. Based on the data presented in this paper, and assuming syllogistic correlations, it is theorized that observing a satellite for as long of an arc as possible, and near zenith and horizon locations, would achieve the lowest levels of orbit determination error.

A comparison of the mean run times for the three orbit determination methods is shown in Table 5.4. The methods were ran 50 times for each method and for each trio-capture (referred to by the date of the observation). Although Vallado's Gauss method code consistently converged the quickest of the three, and Curtis' Gauss converged the slowest, the differences in run times are considered negligible for the purposes of this project. However, if such codes were used for 
Table 5.4: Comparison of Performance Times for Orbit Determination Methods.

\begin{tabular}{|c|c|c|c|c|c|c|c|}
\hline Method & $3 / 25$ & $3 / 27$ & $4 / 12$ & $4 / 143: 56$ & $4 / 144: 57$ & $4 / 20$ & Mean \\
\hline Vallado Gauss & 0.00676 & 0.00672 & 0.0065 & 0.00656 & 0.00646 & 0.00674 & 0.00662 \\
\hline Curtis Gauss & 0.0113 & 0.0113 & 0.0113 & 0.0112 & 0.0112 & 0.0117 & 0.0113 \\
\hline double-r & 0.00812 & 0.00832 & 0.00834 & 0.00832 & 0.00868 & 0.00834 & 0.00835 \\
\hline
\end{tabular}

a very large batch of observations, for instance 20,000 for a statistical study, the difference would be substantial. Approximately 1 minute of run time would be saved selecting the double-r method over Curtis' Gauss, even though the two methods converge to the same level of accuracy. It is prudent, however, to recall that the double-r method is capable of handling observations separated by days, whereas Gauss' method is limited to observations occurring within 5-10 minutes of each other[29].

For the case of the double trio-captures, both the results of the initial singlepass orbit determination and the post-EKF and -LS results are shown in Table 5.5. Of the 13 observed trio sets, 1 failed to converge-accuracy statistics for the successfully-converged sets are shown in Table 5.6. For two of the three multi-arc cases (Globalstar M032 and Globalstar M008), the second angle-sets converged to unrealistic orbits. Because of this, the EKF and LS methods experienced significant error when the state vector results of the first angle-set were used as the initial state epoch. To circumvent this problem, the source TLE's were propagated forward to the time of the first observation, guaranteeing an accurate initial state. For the remaining multi-arc case (Globalstar M058), the angles-only orbit determination results of the first angle-set were utilized as the initial state epoch. Therefore two scenarios are presented: an a priori orbit determination, and an a posteriori orbit determination built upon a preexisting TLE-derived state.

As seen in the tables of this section, large errors exist primarily in the determi- 
Table 5.5: Double Pass Orbit Determination Results.

\begin{tabular}{|c|c|c|c|c|c|c|c|}
\hline Satellite & Source & Epoch (UT) & $A(\mathrm{~km})$ & $e$ & $i\left({ }^{\circ}\right)$ & $\Omega\left({ }^{\circ}\right)$ & $\omega\left(^{\circ}\right)$ \\
\hline \multirow{2}{*}{ Globalstar } & TLE & $5 / 93: 37$ & 8090.2 & 1.90E-4 & 51.996 & 35.54 & 27.07 \\
\hline & Double-R & $5 / 113: 44$ & 7966.0 & $1.38 \mathrm{E}-2$ & 52.042 & 29.97 & 306.39 \\
\hline \multirow{2}{*}{ M058 } & EKF & \multirow{2}{*}{ 5/11 5:47 } & 7974.8 & 1.33E-2 & 52.042 & 29.97 & 322.30 \\
\hline & LS & & 8090.1 & 1.66E-3 & 52.174 & 29.77 & $\mathbf{1 8 5 . 7 2}$ \\
\hline \multirow{2}{*}{ Globalstar } & TLE & 5/9 19:31 & 7791.7 & 1.65E-4 & 52.002 & 54.39 & 47.32 \\
\hline & Double-R & $5 / 114: 14$ & 8539.2 & $8.24 \mathrm{E}-2$ & 52.460 & 50.08 & 113.62 \\
\hline \multirow{2}{*}{ M032 } & EKF & \multirow{2}{*}{ 5/11 6:10 } & 7789.1 & $8.45 \mathrm{E}-4$ & 51.994 & 50.22 & 62.96 \\
\hline & LS & & 7790.2 & $1.10 \mathrm{E}-2$ & 52.403 & 49.29 & 203.74 \\
\hline \multirow{2}{*}{ Globalstar } & TLE & $5 / 94: 03$ & 7902.1 & 2.12E-4 & 51.996 & 56.69 & 155.37 \\
\hline & Double-R & 5/11 4:27 & 7281.8 & $7.76 \mathrm{E}-2$ & 51.634 & 50.80 & 298.18 \\
\hline \multirow{2}{*}{ M008 } & EKF & \multirow{2}{*}{$5 / 116: 24$} & 7900.3 & 9.13E-4 & 51.991 & 50.83 & 77.94 \\
\hline & LS & & 7900.8 & 5.74E-3 & 52.150 & 50.39 & 200.80 \\
\hline
\end{tabular}

Table 5.6: Mean Accuracy of the Orbit Determination Methods (This table shows the mean percent error for the single trio-capture, double-r results, as well as the mean increase (positive), or decrease (negative), in the accuracy (\% change in error) once the Kalman filter is applied. The error values are $1 \sigma)$.

\begin{tabular}{|c|c|c|c|c|c|}
\hline Method & $\mathbf{A}(\mathbf{k m})$ & $e$ & $i\left(^{\circ}\right)$ & $\Omega\left(^{\circ}\right)$ & $\omega\left(^{\circ}\right)$ \\
\hline double-r Error & $\mathbf{4 8 6} \pm \mathbf{2 9 8}$ & $\mathbf{0 . 0 5 5} \pm \mathbf{0 . 0 3 5}$ & $\mathbf{0 . 2 1} \pm \mathbf{0 . 1 8}$ & $\mathbf{3 . 0} \pm \mathbf{2 . 1}$ & $\mathbf{7 3} \pm \mathbf{5 5}$ \\
\hline EKF Improvement & $\mathbf{4 5 7} \pm \mathbf{3 9 4}$ & $\mathbf{0 . 0 5 3} \pm \mathbf{0 . 0 4 5}$ & $\mathbf{0 . 2 7} \pm \mathbf{0 . 2 4}$ & $\mathbf{0 . 0 6} \pm \mathbf{0 . 0 7}$ & $\mathbf{4 4 . 0} \pm \mathbf{2 5 . 4}$ \\
\hline LS Improvement & $\mathbf{4 9 6} \pm \mathbf{3 2 9}$ & $\mathbf{0 . 0 5 2} \pm \mathbf{0 . 0 3 4}$ & $\mathbf{0 . 0 4} \pm \mathbf{0 . 1 7}$ & $\mathbf{- 0 . 4 7} \pm \mathbf{0 . 3 0}$ & $\mathbf{- 2 3 . 6} \pm \mathbf{1 0 4 . 9}$ \\
\hline
\end{tabular}

nation of the satellites' semi-major axis, eccentricity, and argument of perigee. It is hypothesized that these errors are due in part to the relatively short arc of the satellites' orbits that are observed; the angle-sets are separated by 1.5-8.5 minutes and cover $6.6 \%-9.5 \%$ of the satellites' orbital periods. Although large error values accompany the results of the EKF and LS (only three data sets were used), it is apparent that the use of the these techniques at least partially improved the accuracy of the orbit determination-3+ trio-captures would certainly continue to improve the COE's, and such an exercise could prove worthwhile at demonstrating the potential for high-fidelity orbit determination. From the limited data, it is apparent that the extended Kalman filter provided marginally better improvement than the least squares method - the EKF also operated faster than the LS by about an order of magnitude. For the cases in which the source TLE was used for generating the initial state, it is apparent that these methods could 
Table 5.7: Circularization of Orbit Determination Results with use of Fmincon (Globalstar M031).

\begin{tabular}{|c|c|c|c|c|c|c|}
\hline Source & $\mathbf{A ~ ( k m )}$ & $e$ & $i\left(^{\circ}\right)$ & $\Omega\left(^{\circ}\right)$ & $\omega\left(^{\circ}\right)$ & $\theta\left(^{\circ}\right)$ \\
\hline Original Determined & $\mathbf{7 0 4 1 . 7}$ & $\mathbf{1 . 0 5 E - 1}$ & $\mathbf{5 2 . 3 9}$ & $\mathbf{3 3 0 . 1}$ & $\mathbf{3 2 4 . 6 7}$ & $\mathbf{1 8 0 . 3 5}$ \\
\hline Optimized & $\mathbf{7 7 7 9 . 6}$ & $\mathbf{1 . 2 7 E - 5}$ & $\mathbf{5 2 . 3 9}$ & $\mathbf{3 3 0 . 1}$ & $\mathbf{1 0 9 . 2 9}$ & $\mathbf{3 5 . 7 3}$ \\
\hline TLE & $\mathbf{7 8 5 6 . 9}$ & $\mathbf{3 . 0 9 E}-4$ & $\mathbf{5 2 . 0 0}$ & $\mathbf{3 2 9 . 5}$ & $\mathbf{1 4 5 . 1 0}$ & $\mathbf{3 5 7 . 9 3}$ \\
\hline
\end{tabular}

potentially be used to continuously update the COE's with reasonable accuracy.

Additional improvement could focus on providing better estimates of covariance and process noise matrices, to potentially increase the effectiveness of the Kalman filter.

In a final attempt at improving the results of orbit determination, sans additional EKF iterations, MATLAB's fmincon function was used to effectively "circularize" the generated orbit. By constraining the optimizer to maintain angular momentum to within $10 \%$ of its initial value, and optimizing for a minimum eccentricity value, a much more accurate set of COE's were generated. This process, handled by the code orbit_det_withOPT.m, is predicated upon the knowledge that the satellite of interest has a very low eccentricity (0.0001), limiting its potential use for a priori blind-search observations. Results of this optimization exercise are shown in Table 5.7. From this example, it is clear that such a simple optimization of the orbit's eccentricity can greatly improve certain orbital elements (e.g. semi-major axis, argument of perigee, and true anomaly) while holding the remaining elements constant.

\subsection{Temporal TLE Error Growth}

Nine satellites were observed multiple times across several days - the calculated, modulated cross-track errors are shown in Fig. 5.2. The level of CTE seen in the acquired observations far exceeds that shown in Fig. 2.4 taken from Ma- 


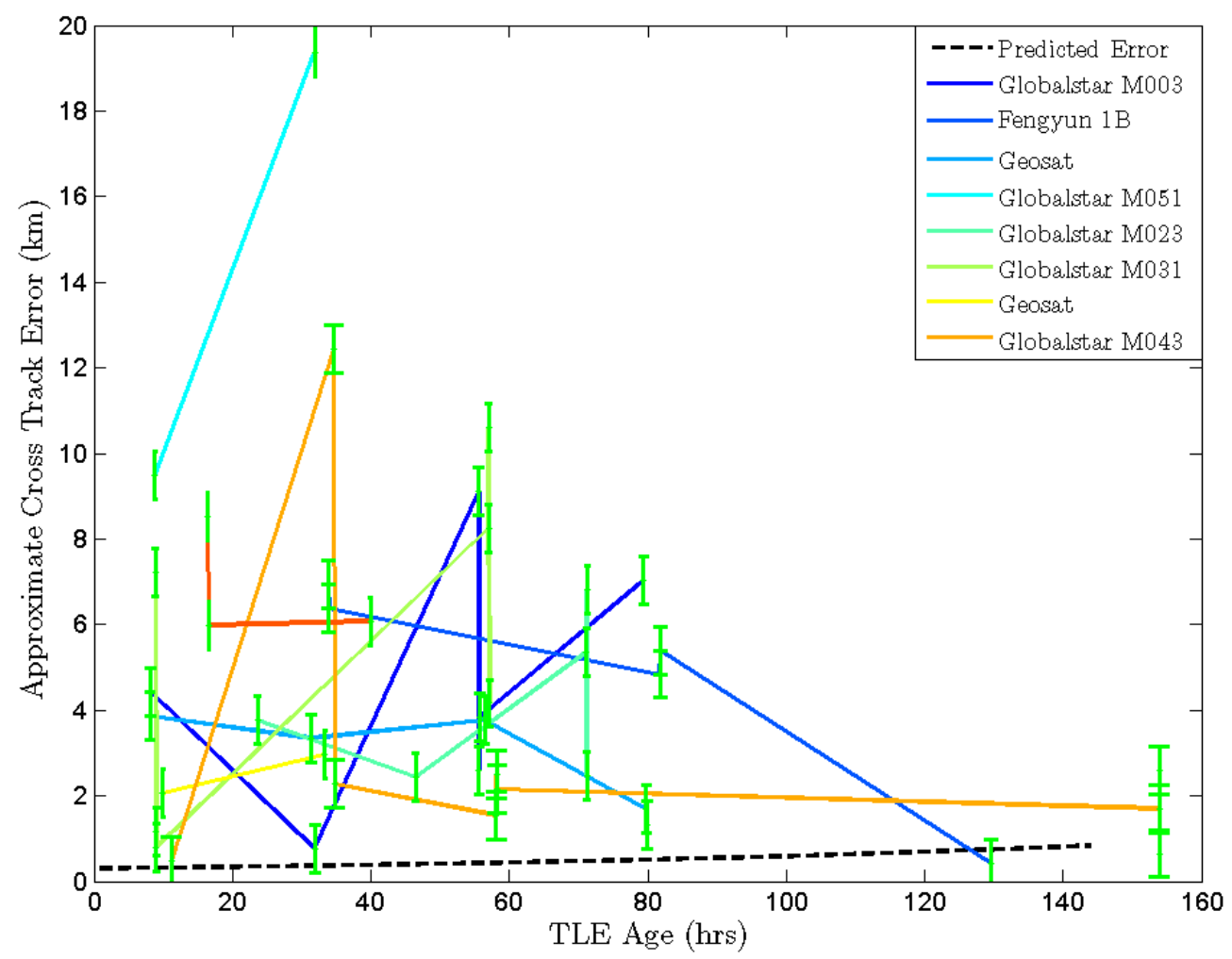

Figure 5.2: Cross-Track Error Results.

son 2009[15]. For a $1000 \mathrm{~km}$ altitude satellite passing directly overhead, a $15 \mathrm{~km}$ cross-track error corresponds to a $\sim 0.86^{\circ}$ angular error. To provide a CTE model, a linear regressive fit to each of the satellites' growth was applied, resulting in Eq. 5.1a.

$$
\begin{array}{r}
\text { Mean }: C T E=0.054 \times T \text { LEage }+4.44 \\
\text { Mean }+1 \sigma: C T E=0.204 \times T \text { LEage }+7.18
\end{array}
$$

Because the uncertainty in calculated CTE- uncertainty due to the pixel selection process (shown as error bars in Fig. 5.2) - is too low to account for the excessive CTE, it is likely that some unknown source of error might be responsible. However, in the event that the results accurately describe the level of CTE that should be expected when using the observation techniques discussed in this 


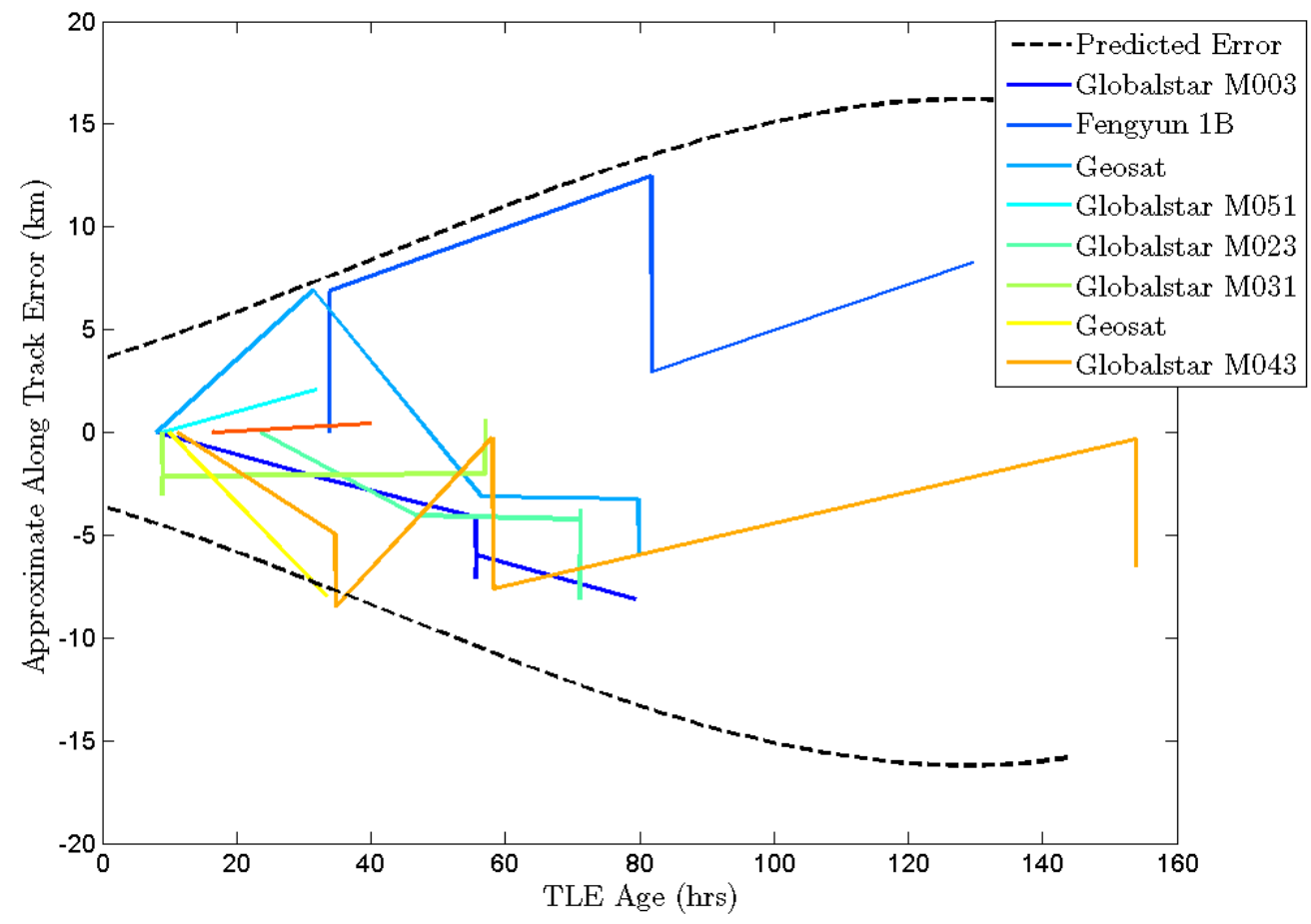

Figure 5.3: Along-Track Error Results.

paper, it remains relevant to apply Eq. 5.1a to the telescope optimization portion of this project.

As for the case of along-track error, the results were much closer to the levels predicted in Fig. 2.4. But due to the unknown levels of error within the timestamps used to calculate ATE, and the assumptions mentioned in Section 4.2.3, the validity of these results are questionable. That being said, these results may warrant a reduction in the recording time margin ( \pm 30 seconds) used in this project. Considering the case of the lowest-altitude satellite observed during this project-IMS-1 at $627 \mathrm{~km}$ - an ATE of $40 \mathrm{~km}$ present during an overhead pass, would result in a timing error of only 5.3 seconds. For a $1000 \mathrm{~km}$ altitude satellite, a $15 \mathrm{~km}$ along-track error corresponds to a $\sim 2$ second timing error. The calculated ATE's are shown in Fig. 5.3. 


\subsection{Magnitude Prediction Model}

To evaluate the accuracy of the magnitude prediction model, it is compared to the value predicted by N2YO. Because the magnitude prediction model produces both a low and high estimate, the average of the two values will be used for the purpose of evaluation. As the N2YO prediction is provided irrespective of the satellite's elevation in the night sky, it is shifted according to the change in observer-satellite range and atmospheric extinction. Additional analysis of the telescope limiting magnitude estimation, provided by Eq. 4.13a, is also included in the results shown in Fig. 5.4. The variation seen in the telescope limiting magnitude is caused by a term left out of Eq. 4.13a, a reduction proportional to the percent of the moon illuminated, found to be a maximum of $-2.9 \mathrm{~V}$ magnitude reduction for a full moon[21]. But because the effect of the moon is also dependent upon the unknown separation distance between the satellite and the moon, the maximum magnitude reduction is estimated to be -2 for the purpose of this paper. The margin of error given to the photometric results are the $1 \sigma$ variation seen within the set of images for a single satellite observation. This variation is likely due to uncertainties in the calculation of noise background within each image, as any error in the noise background will disproportionately affect the amount of light attributed to the satellite vs. the companion star.

A zoomed view of the last 15 observations are shown in Fig. 5.5. Overall, the magnitude prediction model outperformed N2YO; on average, the prediction model used by this paper had an error of 0.93 magnitudes $(\sigma=0.75)$, while the N2YO predictions were off by 1.14 magnitudes $(\sigma=0.79)$. Furthermore, of the 96 observations, this paper's model provided a prediction within the photometric margin of error 21 times, as opposed to 14 times for N2YO. These results demonstrate the validity of developing simple magnitude prediction models, the 


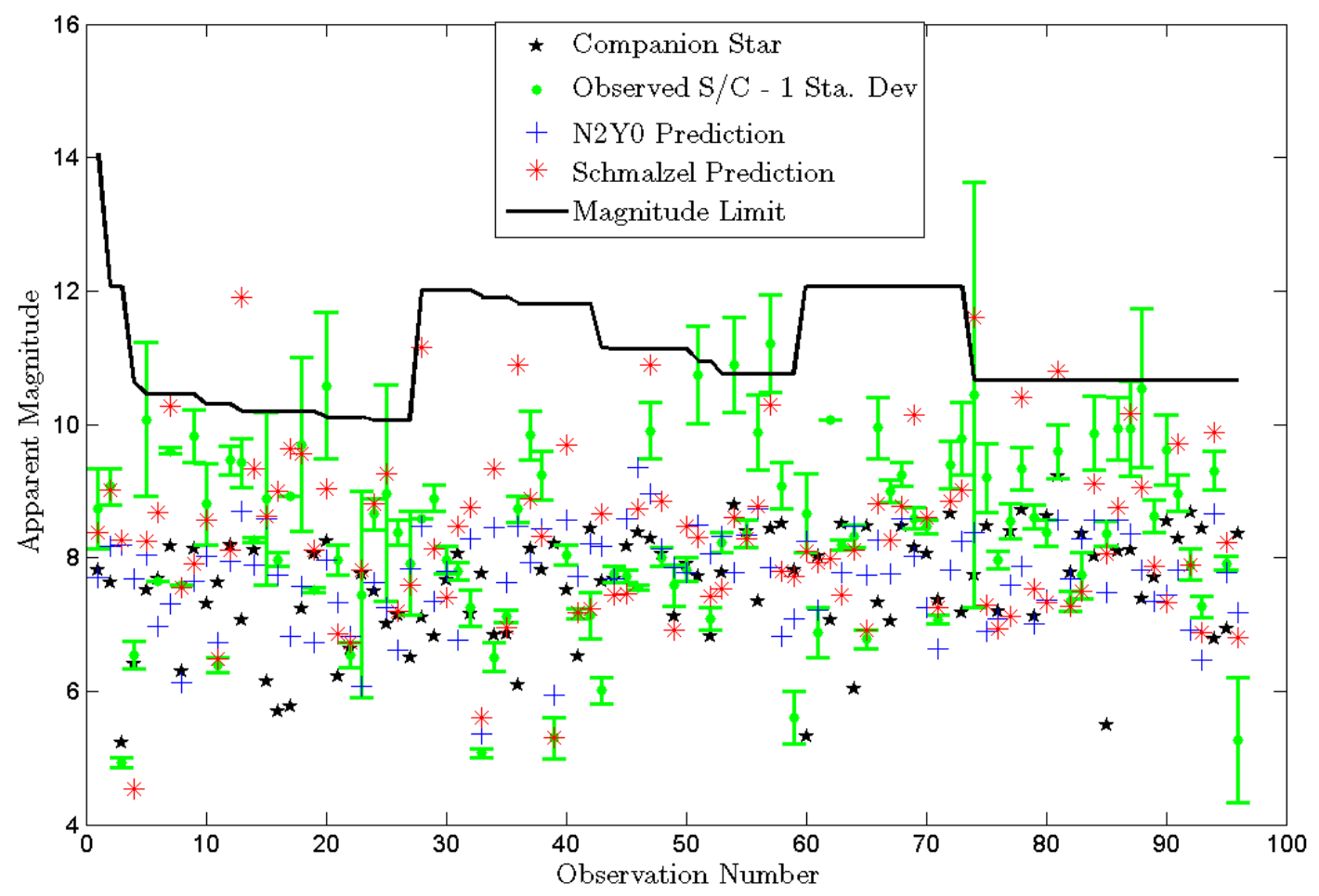

Figure 5.4: Magnitude Prediction Model Results.

likes of which can surpass online resources such as N2YO or Heavens Above. It is also hypothesized that the prediction model would have likely performed better had dimension values for the Globalstar satellites (a majority of the successful observations) been known.

An additional takeaway from this section, is the extent to which the theoretical telescope limiting magnitude accurately describes the actual limiting magnitude. But due to the fact that failed observation attempts can't be attributed entirely to insufficient satellite brightness, as a pointing error may be responsible, it is difficult to provide a complete statistical analysis. Of the 96 successful observations, the upper error bars of 16 observations came within 0.5 magnitudes of the predicted limiting magnitude of the telescope. Considering that the pragmatic limiting magnitude is lower than the theoretical limit, it is likely that a reduction 


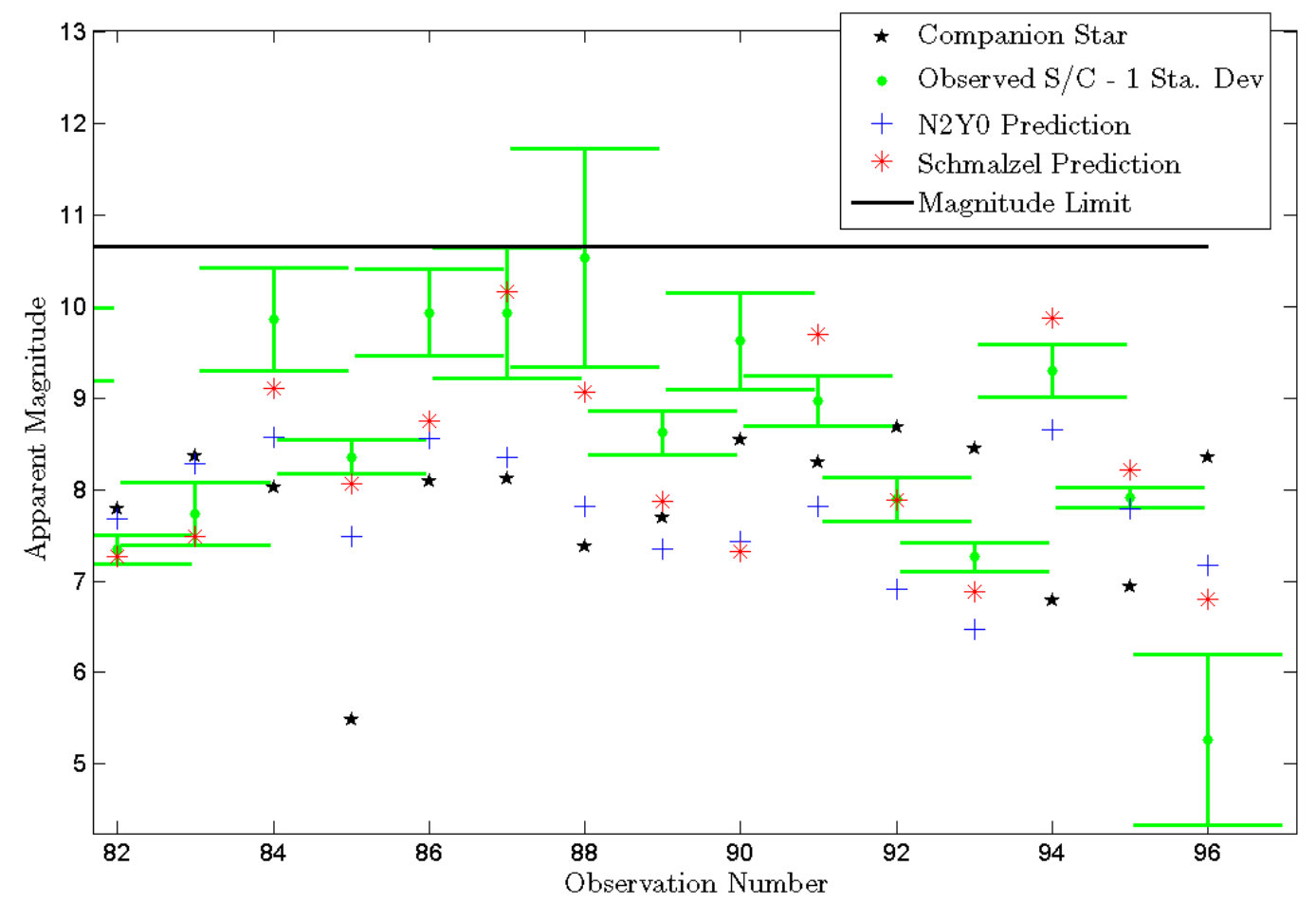

Figure 5.5: Magnitude Prediction Model Results - Last 15 Observations.

factor such as the camera exposure, focal reduction, or moon effect, is excessive. With this in mind, and considering the predictions made in Table 2.1, the magnitude limit seen in Fig. 5.4 suggests that a normal cubesat is likely observable under ideal conditions (e.g. new moon, or low satellite geometrical phase angle).

\subsection{Telescope Optimization}

Three test cases were presented to the telescope fmincon optimizer: CP5 cubesat, IMS-1 $\left(L_{C}=0.8485 \mathrm{~m}\right.$ and $\left.\mathrm{h}=627 \mathrm{~km}\right)$, and Servis-2. For each of these satellites, the optimizer was ran for both levels of temporal error growth shown in Fig. 2.4 (slow) and Eq. 5.1a (fast). The results of these test cases are given in Table 5.8 - each of the optimized telescopes used a 0.2 focal reducer. Visualizations of the optimized telescope dimensions for the IMS-1 case, along with the 


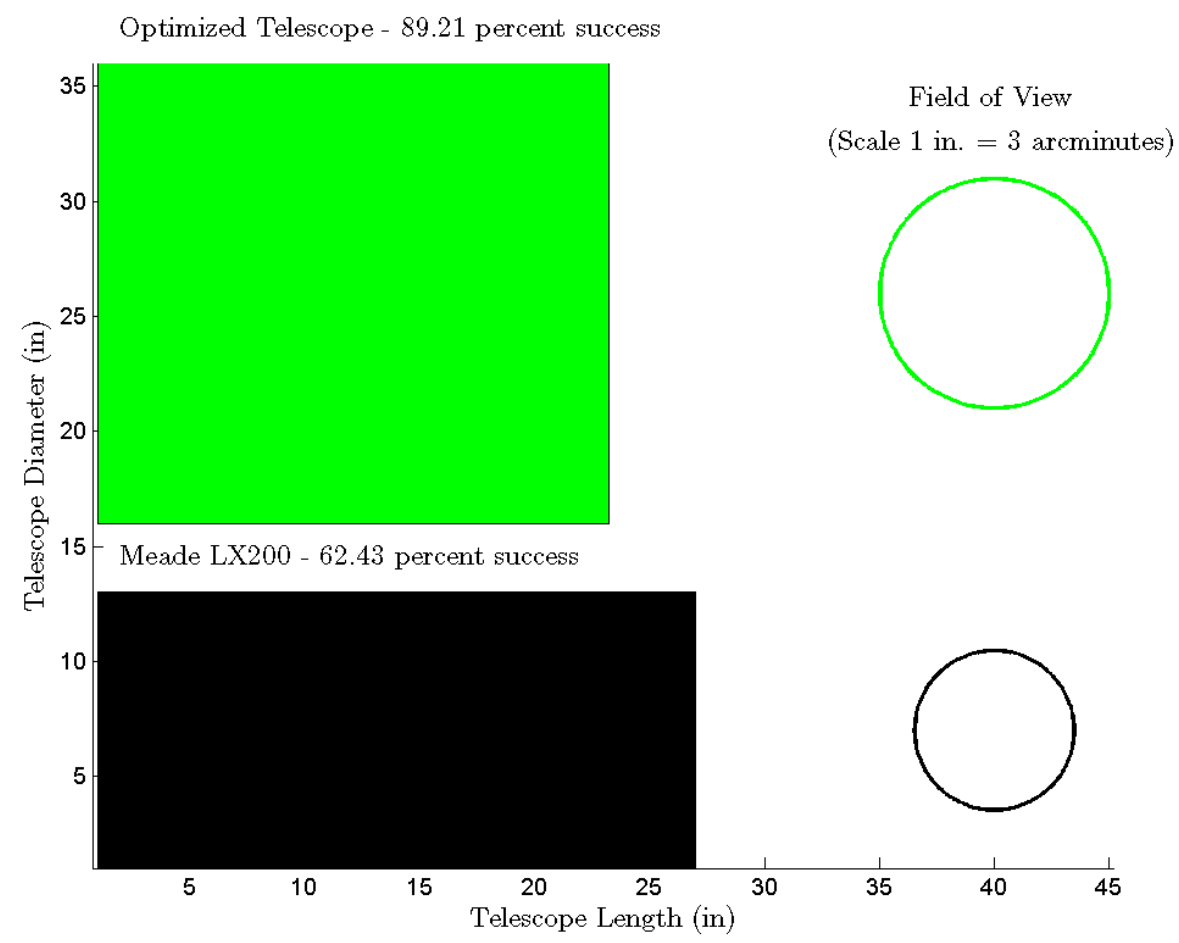

Figure 5.6: Telescope Optimization — IMS-1, Slow Temporal Growth.

FOV size, are shown in Figures 5.6 and 5.7, in comparison to the Meade LX200 telescope used for observations in this project. Because all test cases involved in this section were simulated satellite passes, all magnitude predictions were calculated for an elevation of $45^{\circ}$, a solar phase angle of $90^{\circ}$, and a TLE age of 5 days.

While it appears that the probabilities of a successful capture for CP5 contradict the findings of the previous section, it is likely that the probability would increase significantly for a higher elevation observation and/or lower SPA. Having considered specific test cases, the next step involved analysis of a large, diverse population of 2400 satellites. Ranging from picosatellite classification (0.1-10 $\mathrm{kg})$ to large satellites $(1500-4500 \mathrm{~kg})$, target objects were tested at altitudes of 400-2000 km. Fig. 5.8 and Fig. 5.9 show the results of this analysis, for the 


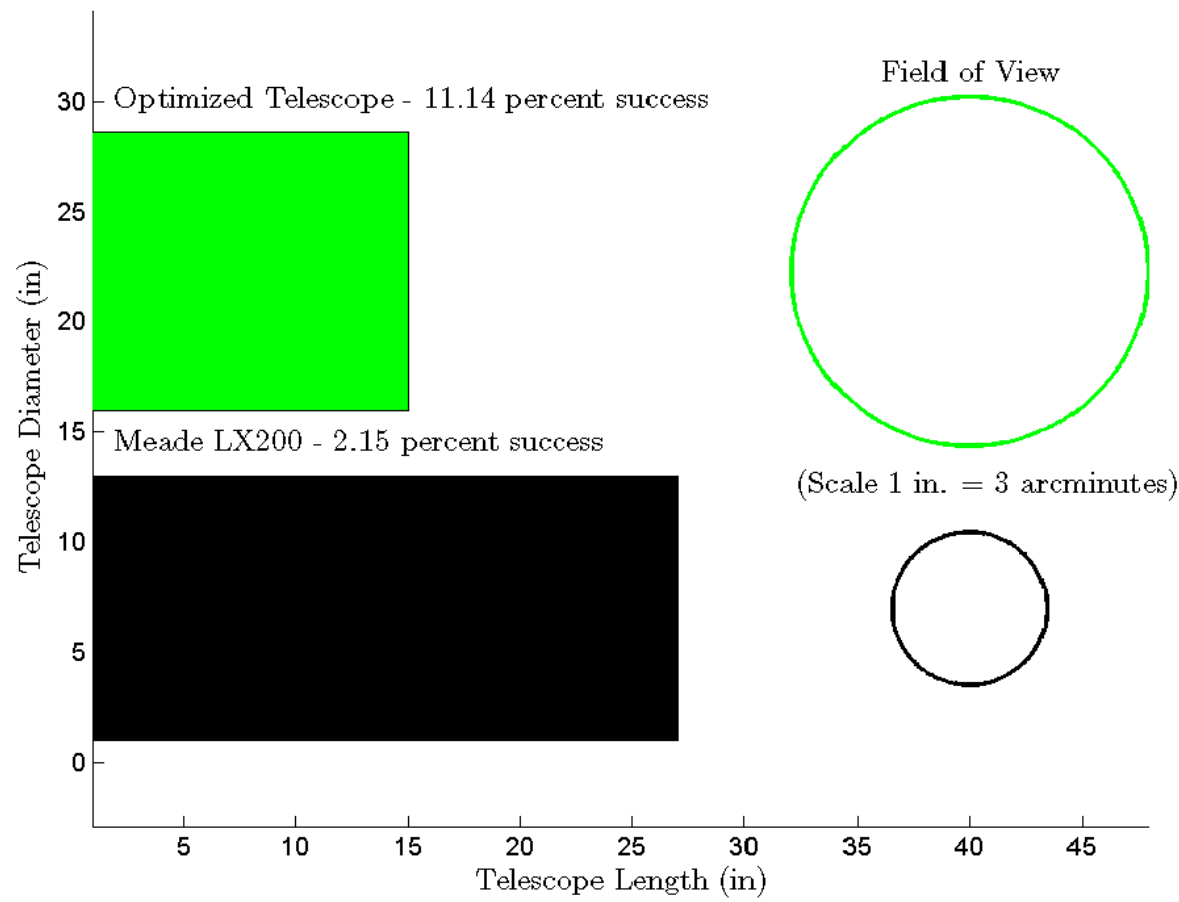

Figure 5.7: Telescope Optimization — IMS-1, Fast Temporal Growth.

Table 5.8: Telescope Optimization for three test cases. $\mathrm{S}=$ slow temporal growth rate, $\mathrm{F}=$ fast temporal growth rate.

\begin{tabular}{|c|c|c|c|c|c|c|}
\hline \multirow{2}{*}{ Satellite } & \multirow{2}{*}{$L_{C}(\mathbf{m})$} & \multirow{2}{*}{$h(\mathbf{k m})$} & \multirow{2}{*}{$\begin{array}{c}D_{T} \text { (in) } \\
\mathbf{S} / \mathbf{F}\end{array}$} & \multirow{2}{*}{$\begin{array}{l}\text { Scope Length } \\
\text { (in) } \mathrm{S} / \mathrm{F}\end{array}$} & \multicolumn{2}{|c|}{ Probability of Capture (\%) } \\
\hline & & & & & Optimized S/F & Meade LX200 S/F \\
\hline & & & $20 / 12$ & $25.4 / 26$ & $0.77 / 0.027$ & $0.05 / 0.003$ \\
\hline IMS-1 & & 627 & $20 / 12.6$ & $22.2 / 14$ & $89.21 / 24.13$ & $62.43 / 0.027$ \\
\hline Servis 2 & 2.6926 & 1207.7 & $20 / 12.6$ & $25.4 / 14$ & $99.89 / 54.29$ & $98.44 / 14.28$ \\
\hline
\end{tabular}




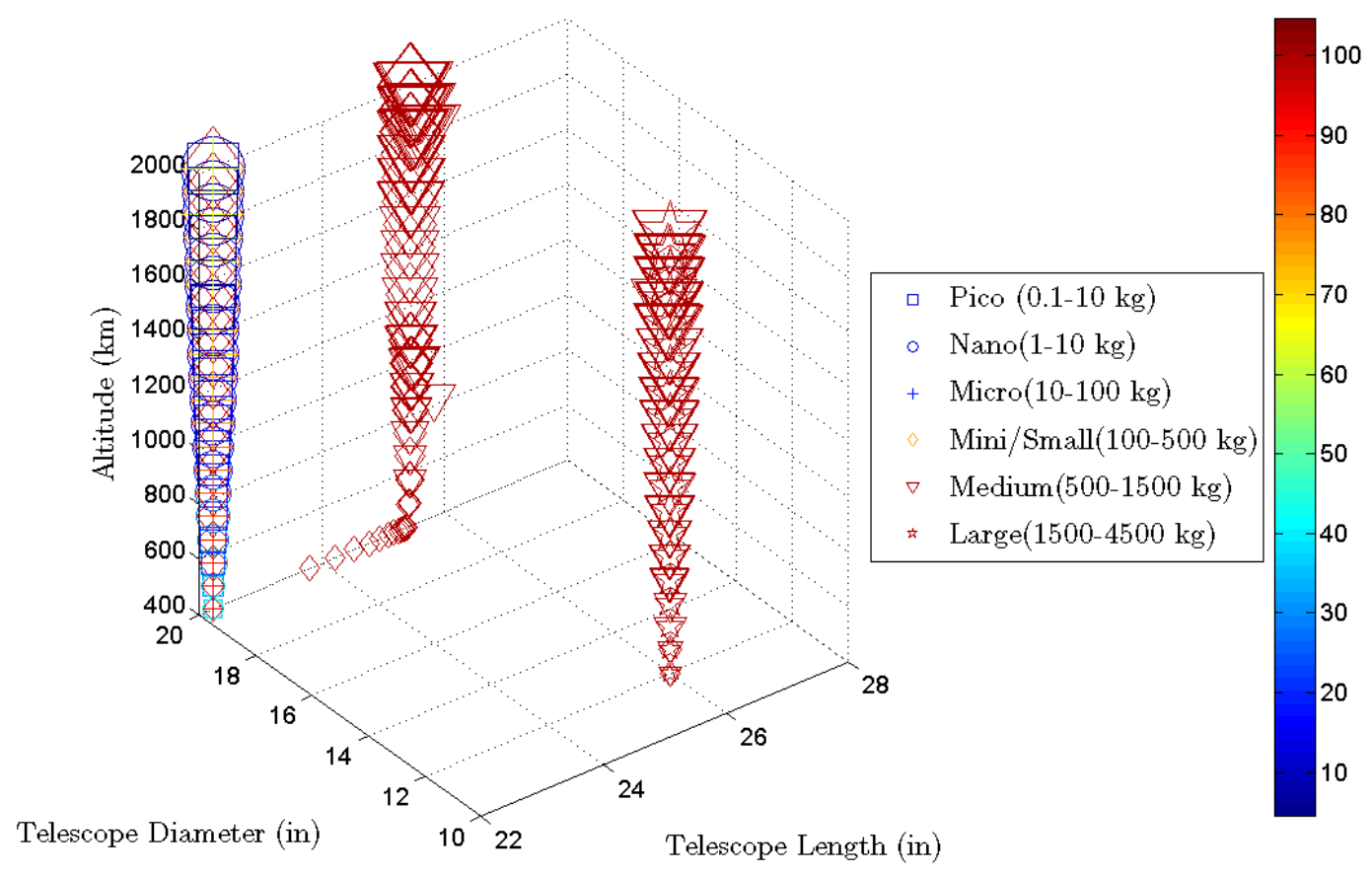

Figure 5.8: Telescope Optimization - Full Population, Slow Temporal Growth. The colorbar indicates the probability of a successful observation, and the size of each marker is proportional to the mass of the satellite (within its own category)

two temporal growth rates. A linear relationship between the satellite mass and satellite characteristic length was utilized, and was calculated from 11 satellites within these mass classifications,

$$
L_{C}=0.0052 m_{a}+0.3128
$$

Satellites appear to cluster around specific sizes of telescopes, suggesting an ideal size for observing a wide range of targets. For the slow temporal growth rate, the larger satellites (Mini-Large) are best obvserved with either a 20 x 25.5 (f/5.74) or 12 x 26 in. (f/9.75) telescope, while a 20 x 22.2 in. (f/5.0) telescope is selected for the smaller satellites (Pico-Mini). When the fast temporal growth rate was used, clusters existed at $\sim 12-12.5 \times 14$ in. (f/5.04) for the larger satellites (Mini-Large), at $20 \times 22.2$ in. (f/5.0) for the smallest satellites (Pico-Nano). 


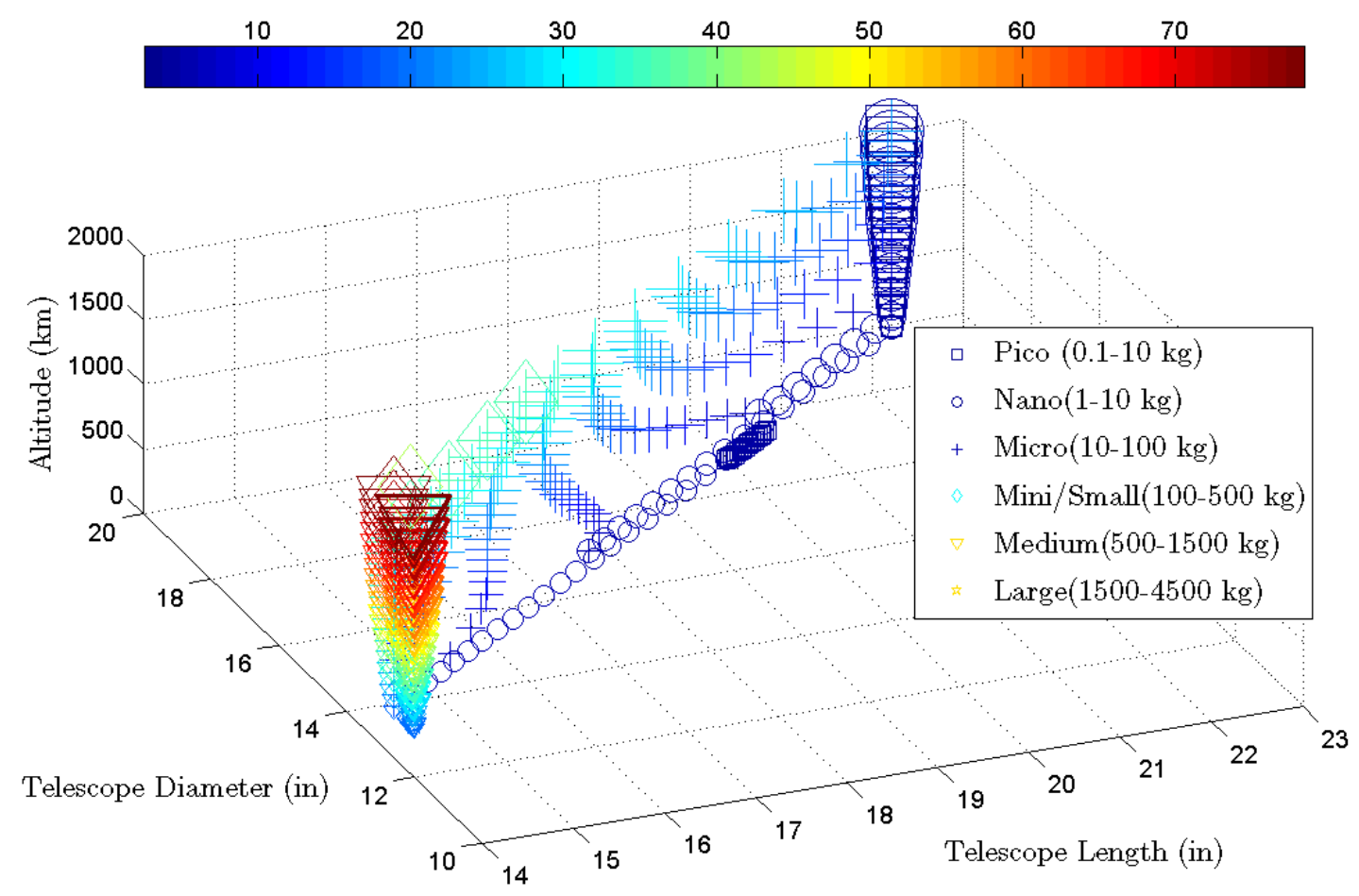

Figure 5.9: Telescope Optimization — Full Population, Fast Temporal Growth. The colorbar indicates the probability of a successful observation, and the size of each marker is proportional to the mass of the satellite (within its own category).

However, many ideal dimensions for Nano and Micro-class satellites were found along the $\mathrm{f} / 5$ line connecting the two clusters. The commercial telescope most closely matching these sizes are listed in Table 5.9. Because the type of telescope often determines the focal length to telescope length ratio, a matching focal ratio does not always mean a matching telescope length. And although these telescopes match the optimized dimensions, their large size (Dobsonian type telescopes) may inhibit their ability to accomplish satellite observations.

It is clear from Table 5.8 that an optimized telescope is capable of providing a better probability of observational success, for both slow and fast rates of temporal TLE error growth. Should a researcher or institution seek a telescope for observing satellites of a certain size, the utilization of a telescope optimizer 
Table 5.9: Commercial Telescopes matched to Optimization Results.

\begin{tabular}{|c|c|c|c|}
\hline Cluster SFR & Commercial Telescope & Telescope Dimensions & Cost (\$) \\
\hline f/9.75 & Meade LX200 & 12 in. f/10 & $\$ 4500$ \\
\hline f/5.0 & Obsession 20 & 20 in. f/5 & $\$ 7200$ \\
\hline f/5.04 & Obsession 12.5 & 12.5 in. f/5 & $\$ 3500$ \\
\hline
\end{tabular}

can be both advantageous and money-saving. 


\section{CHAPTER 6}

\section{CONCLUSIONS}

Given the open-ended nature of this thesis' objective - to demonstrate that amateur telescopes can provide observational data relevant to aerospace researchit is difficult to provide a definitive, quantifiable conclusion. As much of this project's efforts to provide relevant data are considered preliminary endeavors, the quality of data obtained is admittedly mediocre. But considering the quality of equipment used, and the relative simplicity of the observation method, the investigations within this report can be considered successful as many promising results were obtained. Although the orbit elements determined through the use of angles-only observations would not provide the accuracy necessary for guidance, navigation, and control operations, the demonstration of the extended Kalman filter shows promise. If one or more amateur telescopes could provide repeated observations of a satellite, it is likely that an accurate set of COE's could be calculated. Furthermore, it was demonstrated that accurate COE's could be determined through the use of TLE-derived initial states - upon which the gathered data was built upon - and through the use of state optimization (example of eccentricity minimization).

As for the temporal error growth, magnitude prediction, and telescope optimization investigations, the results speak to the question concerning the use of amateur telescopes to target low-priority objects. Given the observed temporal growth of CTE, it is evident that satellites with aging TLE's (at least up to 6 days old) are still observable within a reasonable FOV available to an amateur 
astronomer. In addition, the results of the magnitude prediction model suggest that small satellites, such as cubesats, may be viable targets under ideal observing conditions. Unfortunately, of the few attempts to image microsatellites (10-100 kg), only one was successful: the 0.6 x $0.6 \mathrm{~m}, 83 \mathrm{~kg}$ IMS-1 satellite. But if a cubesat were to be observed under the exact same conditions that IMS-1 was found to have an apparent magnitude of 7.5-7.7, it would be expected to be within the range of 9.5-9.7 magnitude - this prediction would be located $\sim 1.4$ below the telescope's limiting magnitude.

The IMS-1 satellite is a prime example of the results seen within this paper: although the generated data is limited in quality and quantity, it has provided information that could improve and inform future research efforts. All things considered, this paper has successfully demonstrated that it is indeed feasible to generate applicable astrometric and photometric data, with the use of low-cost, amateur-sized telescopes.

Considering the preliminary nature of these applications, it is useful to act under the assumption that another student or researcher could advance and improve the investigations carried out in this project. The process, results, and analytical tools detailed in this paper act as useful precursors to future research.

\subsection{Recommendations for Future Research}

This section details several suggestions for future research that builds upon the material covered in this thesis - any of these recommendations might provide challenging research for a Senior Project or Master's thesis. 
Orbit Determination

As mentioned in the orbit determination section of the Results chapter, further analysis of the usefulness of the extended Kalman filter is recommended. Should $3+$ consecutive satellite passes be captured, additional iterations of the EKF and LS can be initiated. Should the accuracy of the generated COE's continue to improve with each iteration, a significant step towards validationvalidation that orbit determination with amateur telescopes is feasible - will have been taken. It could also be possible to demonstrate that continuous updates to a satellite's COE's, through the use of an EKF, whether or not large perturbations to a satellite's orbit could be absorbed accurately.

It may also prove worthwhile to continue the investigation into the selective optimization of the obtained COE's. Does such an a posteriori circularization provide a consistent improvement to orbit determination? Could other orbital elements apart from eccentricity act as a variable from which to optimize the orbit?

Optical Fence

Given the difficulty in observing such objects as cubesats that push the boundaries of telescopes' limiting magnitudes, a multiple-telescope optical fence may provide a solution. Because telescope magnification power and FOV are inversely related, it is likely that a telescope designed to observe low-magnitude objects may suffer from failed observations caused by CTE. However, if two telescopes could be aligned perpendicular to a satellite's projected direction of movement, with some crossover, an extended FOV can be obtained. In anticipation of such an operation, the MATLAB code scopefence.m was developed to, when given a satellite's projected path and N number of telescopes, provide N RA/Dec target 
angles for the telescopes. As a 14 in. Celestron telescope is housed next door to the Cal Poly Dome Observatory, an attempt at enacting an optical fence is a challenging but real possibility. Additional operations could include using the optical fence to identify any significant CTE as the satellite passes through the first observed point of its arc-the telescope best suited for accurate slewing could then correct for the CTE and continue subsequent observations at the second and third points of the satellite's arc. This would permit operators to devote less resources to controlling the secondary telescopes.

Magnitude Prediction Model/Photometry

There are a few possible additions to the photometric analysis and the magnitude prediction model that could improve the fidelity of the associated results. The magnitude prediction model would benefit greatly from a more accurate sizing model for satellites of unknown dimensions. A simple approach could focus on providing a statistical relationship between the size and mass of satellites of known dimensions, for a greater number of satellites than the sample used for this project (26). A more involved approach could involve considerations of satellite shapes, densities, and extended objects, such as antennas or solar panels. Further possibilities include providing better estimates for the satellite geometrical phase angle (potentially based on assumptions of the satellite's method of control, e.g. three-axis stabilized) and albedo (satellite's surface materials).

Improvement of the photometric analysis could be accomplished by accounting for companion star color, and the telescopic effect of vignetting: the reduction in brightness near the edges of an image. Because atmospheric extinctions affects stars of different colors by varying amounts, a small error arises in the calculation of a satellite's apparent magnitude. And as a satellite's brightness is found 
from multiple frames within an image set, some in which the satellite is located near the periphery of the frame, any vignetting would negatively affect the mean apparent magnitude. Quantifying the affect of vignetting - if it is found to be an issue - would dictate a change to the image analysis.

Miscellaneous Improvements to Observation Process

Several improvements to the equipment and process used to observe satellites could improve the functionality of the telescope system. As analysis of ATE was limited by a lack of accurate timestamps, the inclusion of a GPS time-inserter could remedy this issue. It may also be beneficial to save the images generated from the camera in FITS format, as opposed to JPEG. This change may allow more traditional image reduction and analysis used by astronomers, with applications such as Image Reduction and Analysis Facility (IRAF) and SAOImage DS9. Switching to FITS format could potentially automate the photometry analysis.

Another potential improvement includes the use of SGP4 propagation within MATLAB, to remove STK entirely from the observation preparation process. This upgrade was previously attempted, but STK-level accuracy was not achieved. If a new attempt were to be successful, the pool of satellite target candidates could be increased (as much preparation time would be saved by bypassing STK). Presenting a larger number of potential targets could improve aspects of observational research.

For the observations in which a focal reducer was used, the probability of secondary companion stars increased significantly. It may prove worthwhile to utilize these stars as additional reference points for data analysis. In the process of RA/Dec angle determination, the secondary star(s) can be used to determine the orientation of the RA/Dec plane, thereby validating the method used in this 
project (alignment of the predicted and actual direction of satellite movement). Furthermore, the additional stars can be used to increase the accuracy of the comparative photometry. 


\section{CHAPTER 7}

\section{APPENDICES}

7.1 Selected Image Sets \& Raw Data

Table 7.1: Observational Results - Astrometry Data (Observational data including the predicted target (Tar.) and actual determined (Det.) RA/Dec angles).

\begin{tabular}{|c|c|c|c|c|c|c|}
\hline Satellite & Tar. RA & Tar. Dec & Det. RA & Det. Dec & CTE (km) & ATE (km) \\
\hline Meteor 3-5 & 186.174 & 23.954 & 186.296 & 24.044 & 2.880 & 6.772 \\
\hline Globalstar M003 & 88.509 & -15.755 & 88.707 & -15.730 & 6.712 & 9.169 \\
\hline Fengyun 1B & 86.966 & -13.443 & 87.322 & -13.385 & 8.818 & 0.492 \\
\hline Fengyun 1B & 32.959 & 48.504 & 33.316 & 48.707 & 10.066 & 7.357 \\
\hline Geosat & 73.587 & 0.430 & 73.857 & 0.481 & 5.548 & 6.226 \\
\hline Globalstar M051 & 204.494 & 66.744 & 204.463 & 66.751 & 1.398 & 3.189 \\
\hline Globalstar M003 & 85.298 & -15.289 & 85.383 & -15.291 & 2.441 & 7.009 \\
\hline Geosat & 135.052 & -27.453 & 135.464 & -27.492 & 9.326 & 13.143 \\
\hline Globalstar M051 & 206.931 & 62.883 & 207.442 & 62.956 & 26.574 & 5.302 \\
\hline Globalstar M003 & 30.193 & 23.345 & 30.381 & 23.525 & 12.636 & 5.028 \\
\hline Globalstar M003 & 65.146 & 0.757 & 65.134 & 0.774 & 0.226 & 2.068 \\
\hline Globalstar M003 & 99.858 & -30.490 & 99.976 & -30.440 & 5.971 & 3.228 \\
\hline Fengyun 1B & 101.013 & -31.106 & 101.293 & -31.148 & 7.507 & 12.949 \\
\hline Fengyun 1B & 49.237 & 43.960 & 49.599 & 44.124 & 8.761 & 3.419 \\
\hline Globalstar M023 & 54.065 & 34.313 & 54.191 & 34.406 & 4.688 & 8.326 \\
\hline Geosat & 77.885 & -10.983 & 78.103 & -10.953 & 5.754 & 3.118 \\
\hline Globalstar M003 & 72.267 & -8.171 & 72.479 & -8.074 & 9.183 & 1.047 \\
\hline
\end{tabular}




\begin{tabular}{|c|c|c|c|c|c|c|}
\hline Satellite & Tar. RA & Tar. Dec & Det. RA & Det. Dec & CTE (km) & ATE (km) \\
\hline Globalstar M023 & 155.706 & -4.020 & 155.968 & -4.049 & 7.737 & 4.282 \\
\hline Geosat & 123.899 & -30.850 & 124.041 & -31.009 & 0.733 & 2.990 \\
\hline Geosat & 53.546 & 44.797 & 53.833 & 44.798 & 2.977 & 0.373 \\
\hline Fengyun 1B & 104.893 & -29.993 & 105.079 & -30.090 & 4.068 & 8.760 \\
\hline Globalstar M023 & 58.632 & 31.006 & 58.886 & 31.141 & 7.732 & 4.102 \\
\hline Globalstar M023 & 102.754 & 5.111 & 102.959 & 5.202 & 5.965 & 0.229 \\
\hline Globalstar M023 & 141.455 & -28.617 & 141.600 & -28.710 & 1.252 & 4.585 \\
\hline Globalstar M031 & 53.513 & 31.950 & 53.874 & 32.082 & 9.315 & 4.919 \\
\hline Globalstar M031 & 96.498 & 13.178 & 96.634 & 13.163 & 2.185 & 1.849 \\
\hline Globalstar M031 & 133.642 & -18.759 & 133.739 & -18.770 & 2.148 & 2.767 \\
\hline Geosat & 70.309 & 49.900 & 70.718 & 49.952 & 3.646 & 14.983 \\
\hline Globalstar M043 & 105.021 & 54.850 & 105.141 & 54.828 & 1.851 & 9.945 \\
\hline Geosat & 182.775 & 64.577 & 183.276 & 64.420 & 7.649 & 6.999 \\
\hline Globalstar M043 & 86.500 & 58.704 & 86.535 & 58.915 & 8.648 & 4.971 \\
\hline Globalstar M043 & 184.914 & 38.003 & 185.010 & 37.998 & 2.698 & 1.480 \\
\hline Globalstar M031 & 65.695 & 16.731 & 65.907 & 16.886 & 11.720 & 2.910 \\
\hline
\end{tabular}

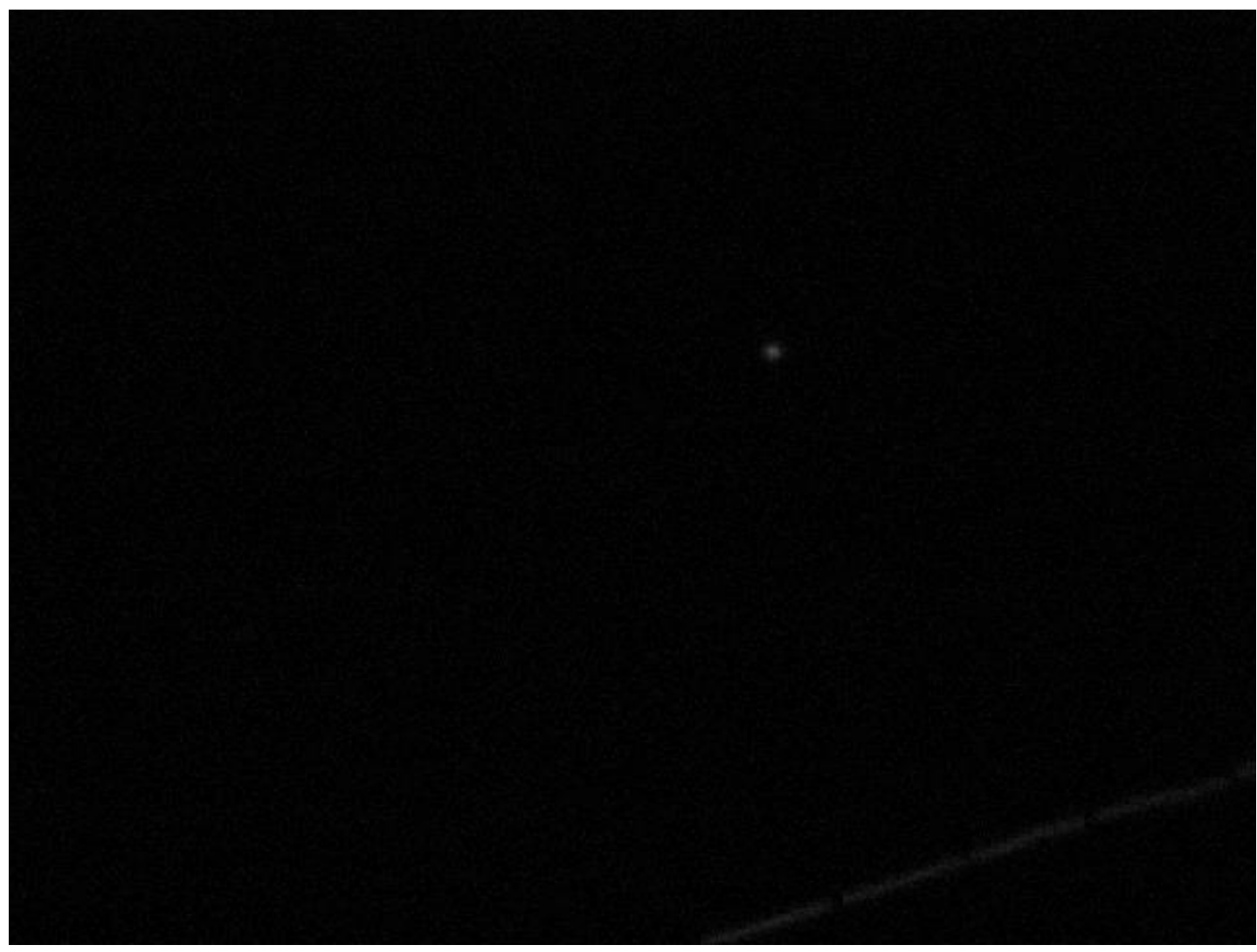

Figure 7.1: Observation of IMS-1. Images taken of the Indian Remote Sensing satellite, IMS-1, on April 17 ${ }^{\text {th }}$, 2013, at 21:18 PST. At $83 \mathrm{~kg}$, this satellite was the least massive of all objects observed. 


\begin{tabular}{|c|c|c|c|c|c|c|}
\hline Satellite & Tar. RA & Tar. Dec & Det. RA & Det. Dec & CTE (km) & $\operatorname{ATE}(\mathrm{km})$ \\
\hline Globalstar M031 & 92.903 & -3.695 & 93.223 & -3.484 & 15.463 & 4.335 \\
\hline Globalstar M031 & 120.966 & -29.104 & 120.977 & -29.167 & 1.874 & 5.563 \\
\hline Cosmos 1900 & 118.142 & 10.713 & 118.409 & 10.606 & 4.520 & 8.256 \\
\hline Globalstar M043 & 72.079 & 61.455 & 72.220 & 61.468 & 4.075 & 9.672 \\
\hline Globalstar M043 & 185.364 & 47.195 & 185.572 & 47.213 & 7.042 & 2.785 \\
\hline Globalstar M043 & 205.087 & 17.857 & 205.298 & 17.787 & 7.473 & 2.320 \\
\hline Globalstar M055 & 114.790 & -29.177 & 114.966 & -29.281 & 9.930 & 3.140 \\
\hline Globalstar M055 & 215.698 & 37.230 & 215.836 & 37.152 & 4.132 & 2.464 \\
\hline Oscar 36 & 199.286 & 50.548 & 199.588 & 50.279 & 5.361 & 6.240 \\
\hline IMS-1 & 213.576 & 44.384 & 213.897 & 44.305 & 5.170 & 7.124 \\
\hline Globalstar M043 & 65.440 & 47.607 & 65.585 & 47.611 & 3.864 & 9.656 \\
\hline Globalstar M043 & 102.123 & 53.008 & 102.245 & 53.069 & 2.892 & 3.429 \\
\hline Globalstar M043 & 180.155 & 12.474 & 180.350 & 12.435 & 5.232 & 3.890 \\
\hline Globalstar M055 & 271.488 & 65.144 & 271.074 & 65.209 & 16.740 & 4.243 \\
\hline Globalstar M053 & 128.166 & -15.724 & 128.418 & -15.804 & 12.269 & 3.268 \\
\hline Globalstar M053 & 206.844 & 42.096 & 206.952 & 41.944 & 7.620 & 3.268 \\
\hline Globalstar M053 & 206.266 & 34.691 & 206.389 & 34.561 & 7.805 & 3.710 \\
\hline Globalstar M036 & 251.705 & 54.436 & 251.600 & 54.519 & 6.026 & 1.680 \\
\hline Globalstar M008 & 259.143 & 62.526 & 259.281 & 62.687 & 8.885 & 0.979 \\
\hline Globalstar M053 & 270.152 & 63.556 & 270.081 & 63.480 & 5.473 & 0.977 \\
\hline Globalstar M036 & 73.981 & 58.002 & 74.402 & 58.089 & 21.631 & 11.370 \\
\hline Globalstar M036 & 245.715 & 62.511 & 245.874 & 62.570 & 6.735 & 5.284 \\
\hline Globalstar M036 & 252.234 & 41.760 & 252.246 & 41.735 & 0.294 & 4.129 \\
\hline Globalstar M050 & 239.421 & 54.776 & 239.485 & $\mathbf{5 4 . 8 3 4}$ & 2.949 & 2.819 \\
\hline Globalstar M036 & 235.805 & 52.908 & 236.339 & 52.705 & 23.926 & 13.437 \\
\hline Globalstar M026 & 150.700 & -33.134 & 151.024 & -33.343 & 17.493 & 13.718 \\
\hline Globalstar M026 & 221.879 & 21.787 & 222.084 & 21.599 & 11.544 & 3.212 \\
\hline Globalstar M028 & 137.310 & -30.269 & 137.653 & -30.489 & 17.739 & 3.170 \\
\hline Globalstar M028 & 216.525 & 28.650 & 216.705 & 28.434 & 9.664 & 1.997 \\
\hline Globalstar M022 & 270.572 & 58.563 & 270.674 & 58.716 & 6.635 & 3.418 \\
\hline Globalstar M026 & 273.679 & 56.609 & 273.545 & 56.637 & 3.659 & 2.080 \\
\hline Globalstar M028 & 290.026 & 56.900 & 290.021 & 57.019 & 1.859 & 0.039 \\
\hline Globalstar M052 & 90.024 & 45.209 & 90.319 & 45.011 & 14.607 & 11.614 \\
\hline Globalstar M052 & 112.536 & 50.149 & 112.726 & 49.923 & 9.177 & 0.210 \\
\hline Globalstar M052 & 145.301 & 48.769 & 145.462 & 48.877 & 4.240 & 2.602 \\
\hline Globalstar M022 & 293.052 & 60.789 & 292.840 & 60.983 & 11.762 & 9.529 \\
\hline Globalstar M022 & 291.321 & 44.920 & 291.661 & 45.015 & 18.719 & 6.457 \\
\hline Globalstar M058 & 62.246 & 53.344 & 62.065 & 53.371 & 8.934 & 0.656 \\
\hline Globalstar M058 & 190.502 & 55.665 & 190.293 & 55.845 & 2.039 & 1.372 \\
\hline Globalstar M058 & 208.357 & 33.803 & 208.218 & 33.985 & 1.688 & 0.258 \\
\hline Globalstar M058 & 218.230 & 13.856 & 218.144 & 14.024 & 0.211 & 1.598 \\
\hline Globalstar M032 & 60.550 & 64.399 & 60.254 & 64.196 & 12.593 & 7.972 \\
\hline Globalstar M032 & 242.155 & 63.417 & 242.513 & 63.615 & 12.921 & 1.035 \\
\hline Globalstar M032 & 246.076 & 37.012 & 246.009 & 37.172 & 1.982 & 5.469 \\
\hline Globalstar M008 & 66.426 & 60.305 & 66.505 & 60.203 & 4.895 & 3.443 \\
\hline Globalstar M008 & 241.459 & 43.707 & 241.237 & 43.861 & 6.942 & 1.587 \\
\hline Globalstar M008 & 246.796 & 21.185 & 246.882 & 21.415 & 6.390 & 1.536 \\
\hline Globalstar M027 & 124.329 & 1.133 & 124.301 & 0.939 & 2.494 & 1.261 \\
\hline Globalstar M027 & 133.923 & 27.879 & 133.814 & 27.728 & 1.803 & 3.673 \\
\hline Globalstar M027 & 296.090 & 55.840 & 296.193 & 56.095 & 9.915 & 3.156 \\
\hline Globalstar M058 & 112.087 & 29.343 & 111.941 & 29.528 & 7.672 & 6.085 \\
\hline Globalstar M058 & 127.343 & 24.324 & 127.243 & 24.471 & 4.270 & 2.144 \\
\hline Globalstar M058 & 149.739 & 10.947 & 149.566 & 10.996 & 2.779 & 3.118 \\
\hline Globalstar M058 & 174.182 & -10.425 & 173.968 & -10.398 & 5.123 & 4.220 \\
\hline Globalstar M032 & 112.089 & 42.802 & 111.907 & 42.662 & 4.344 & 1.717 \\
\hline Globalstar M032 & 142.371 & 43.193 & 142.174 & 43.239 & 0.301 & 3.790 \\
\hline Globalstar M032 & 167.169 & 36.444 & 166.998 & 36.454 & 1.918 & 4.168 \\
\hline Globalstar M008 & 114.298 & 40.110 & 114.150 & 39.919 & 8.302 & 2.768 \\
\hline Globalstar M008 & 140.285 & 39.586 & 140.143 & 39.784 & 6.122 & 2.883 \\
\hline Globalstar M008 & 174.376 & 27.387 & 174.285 & 27.593 & 3.498 & 3.188 \\
\hline
\end{tabular}




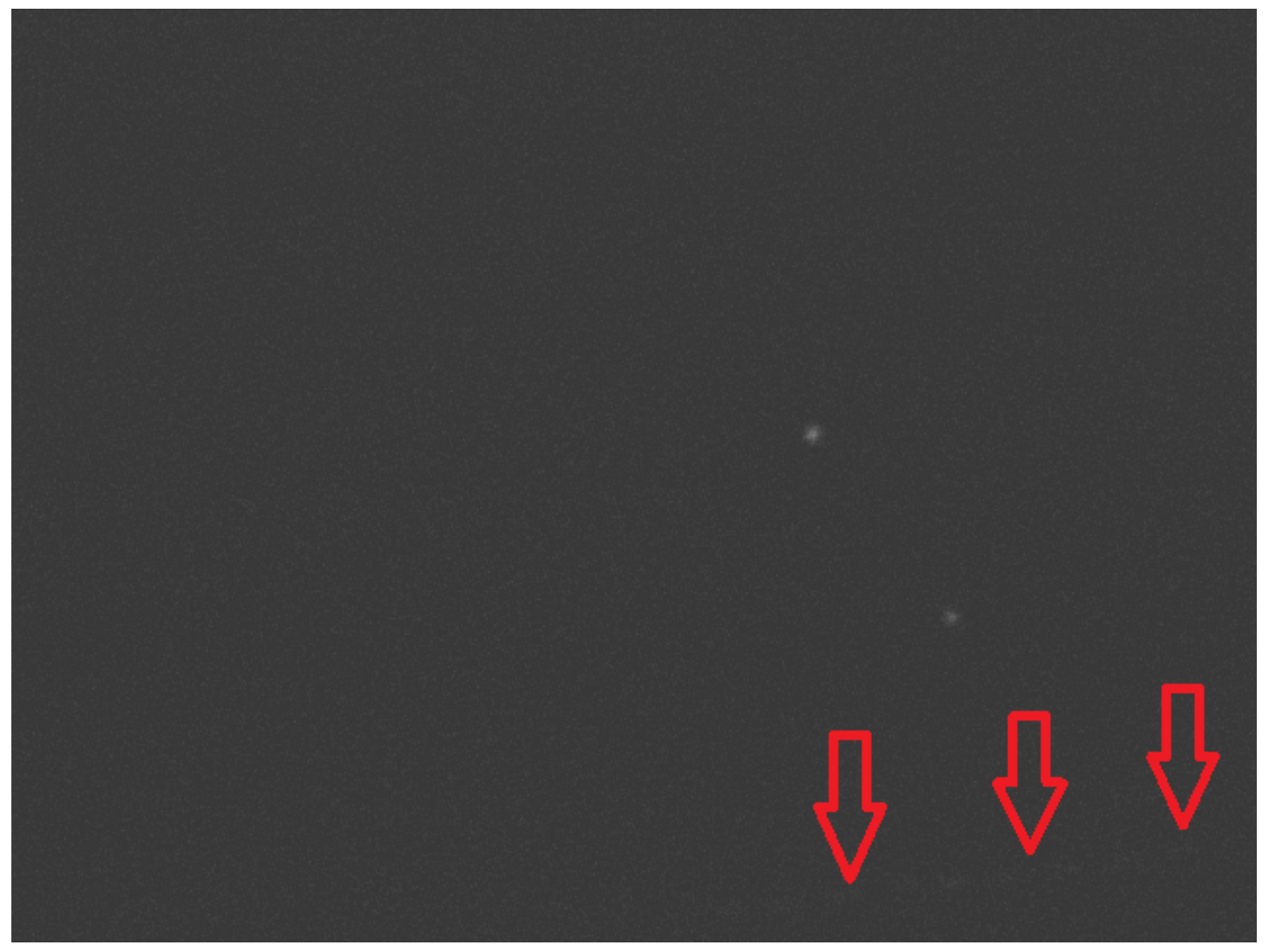

Figure 7.2: Observation of Globalstar M035. Images taken of Globalstar M035, on April 20 ${ }^{\text {th }}, 2013$, at 23:03 PST. At an apparent magnitude of approximately 11.2, this was the dimmest object observed. For the purpose of presentation, every second image from the set was removed, and the brightness and contrast values for the images increased. 


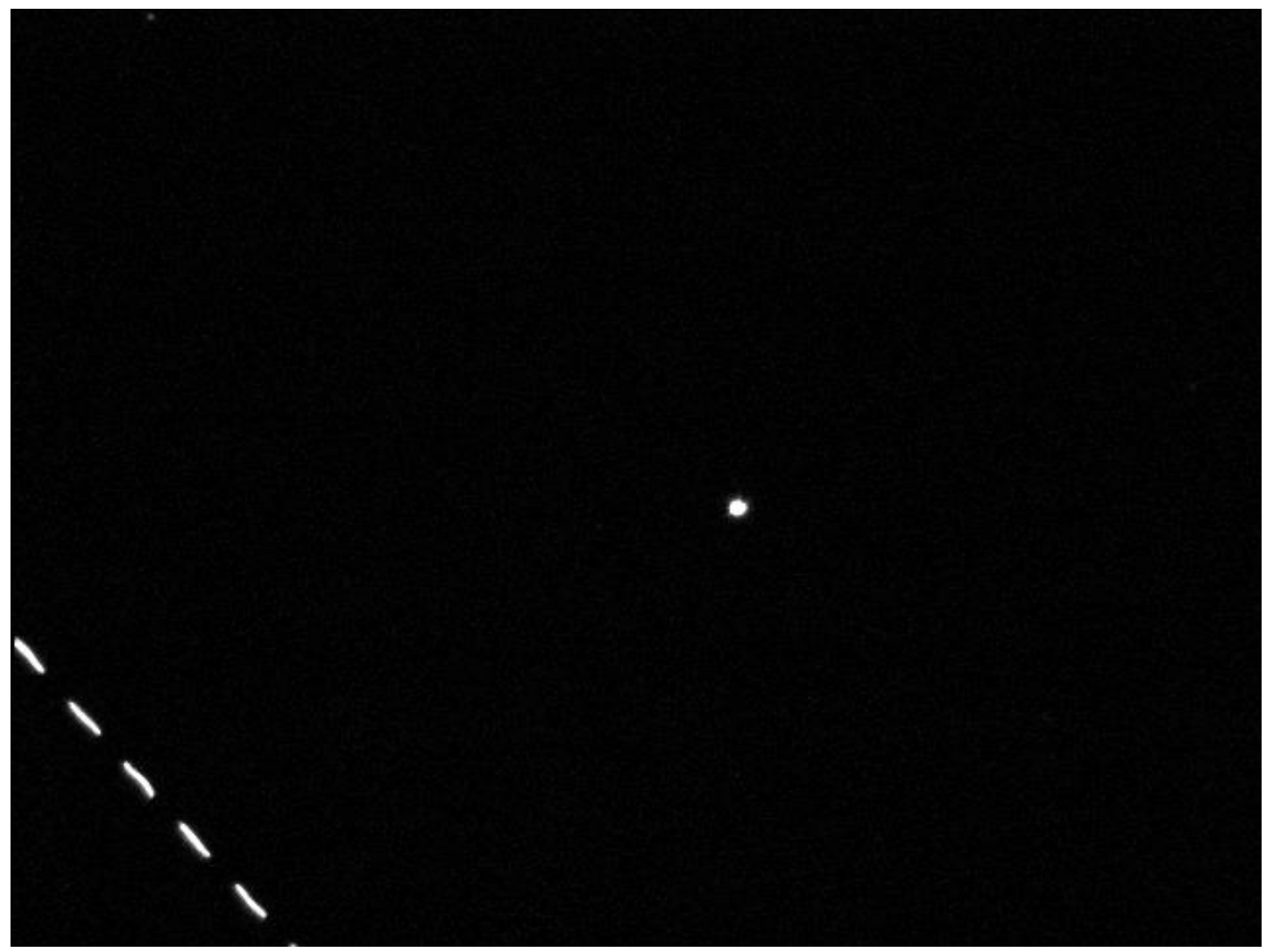

Figure 7.3: Observation of Globalstar M002. Images taken of Globalstar M002, on March 12 ${ }^{\text {th }}, 2013$, at 21:41 PST. At an apparent magnitude of approximately 4.93, this was the brightest object observed. Every second image from the set was removed. 


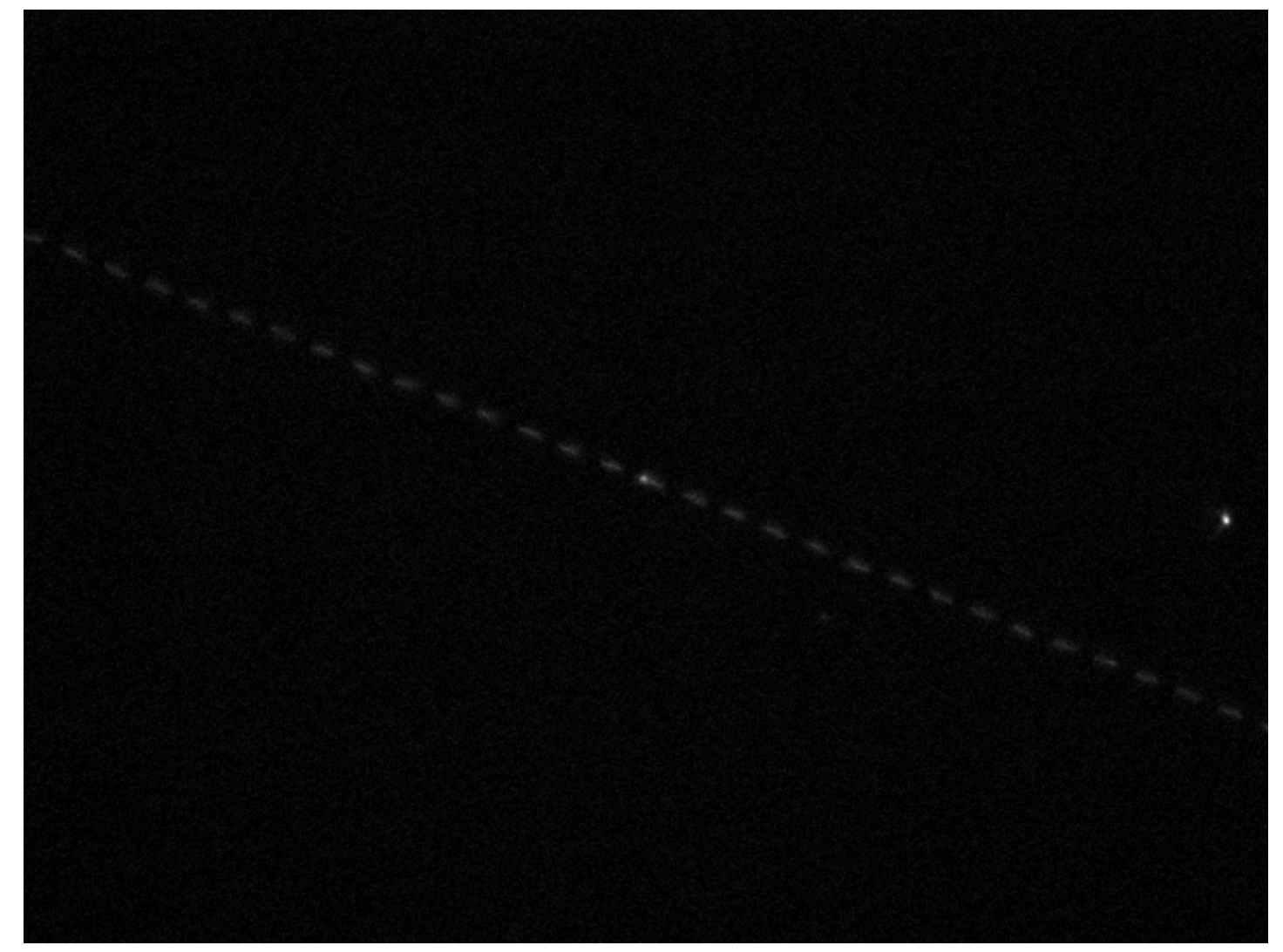

Figure 7.4: Observation of Globalstar M058. Images taken of Globalstar M058, on May 10 ${ }^{\text {th }}, 2013$, at 20:49 PST. This observation had a notably low cross-track error of only $0.21 \mathrm{~km}$ - every second image from the set was removed. 
Table 7.2: Observational Results - Photometry Data Part 1 (Observational data relevant to photometric analysis, where D1 and D2 are dimensions of each satellite in meters (if listed as 0 , dimension was not known), and TLE is the age of the reference TLE).

\begin{tabular}{|c|c|c|c|c|c|c|c|c|}
\hline Satellite & FOV $\left({ }^{\prime}\right)$ & el $\left({ }^{\circ}\right)$ & $R(\mathbf{k m})$ & TLE(hr) & D1 & D2 & Mass(kg) & SPA $\left(^{\circ}\right)$ \\
\hline Globalstar M049 & 4.01 & 44.30 & 2692.00 & 4.79 & 0 & $\mathbf{0}$ & 450 & 89.20 \\
\hline Globalstar M003 & 12.139 & 33.90 & 2458.10 & 8.06 & 0 & 0 & 450 & 111.10 \\
\hline Globalstar M002 & 12.139 & 35.80 & 2676.60 & 8.9 & $\mathbf{0}$ & $\mathbf{0}$ & 450 & 83.70 \\
\hline Meteor 3-5 & 12.139 & 25.30 & 2204.20 & 7.99 & 12.7 & 6.5 & 2150 & 25.00 \\
\hline Globalstar M003 & 12.139 & 32.30 & 2516.70 & 8.16 & 0 & 0 & 450 & 86.60 \\
\hline Fengyun 1B & 12.139 & 32.20 & 1469.30 & 33.81 & 1.8 & 1.4 & 881 & 95.10 \\
\hline Fengyun 1B & 12.139 & 26.90 & 1636.80 & 33.89 & 1.8 & 1.4 & 881 & 125.80 \\
\hline Geosat & 12.139 & 31.50 & 1310.60 & 8.08 & 3 & 0 & 635 & 108.90 \\
\hline Globalstar M051 & 12.139 & 38.90 & 2689.20 & 8.82 & $\mathbf{0}$ & $\mathbf{0}$ & 450 & 69.60 \\
\hline Globalstar M003 & 12.139 & 32.50 & 2507.90 & 31.91 & $\mathbf{0}$ & $\mathbf{0}$ & 450 & 97.40 \\
\hline Geosat & 12.139 & 24.50 & 1527.00 & 31.35 & 3 & $\mathbf{0}$ & 635 & 53.00 \\
\hline Globalstar M051 & 12.139 & 31.90 & 2965.40 & 31.9 & $\mathbf{0}$ & $\mathbf{0}$ & 450 & 66.70 \\
\hline Globalstar M003 & 12.139 & 21.60 & 3041.33 & 55.54 & $\mathbf{0}$ & $\mathbf{0}$ & 450 & 146.90 \\
\hline Globalstar M003 & 12.139 & 35.36 & 2401.43 & 55.61 & $\mathbf{0}$ & $\mathbf{0}$ & 450 & 119.10 \\
\hline Globalstar M003 & 12.139 & 23.19 & 2943.16 & 55.71 & $\mathbf{0}$ & $\mathbf{0}$ & 450 & 84.20 \\
\hline Fengyun 1B & 12.139 & 21.08 & 1874.94 & 81.73 & 1.8 & 1.4 & 881 & 83.28 \\
\hline Fengyun 1B & 12.139 & 35.28 & 1384.10 & 81.83 & 1.8 & 1.4 & 881 & 122.11 \\
\hline Globalstar M023 & 12.139 & 29.46 & 2341.58 & 23.6 & $\mathbf{0}$ & $\mathbf{0}$ & 450 & 123.48 \\
\hline Geosat & 12.139 & 20.65 & 1686.86 & 56.42 & 3 & $\mathbf{0}$ & 635 & 105.70 \\
\hline Globalstar M003 & 12.139 & 34.81 & 2420.25 & 79.39 & $\mathbf{0}$ & $\mathbf{0}$ & 450 & 112.29 \\
\hline Globalstar M023 & 12.139 & 34.79 & 2139.70 & 46.5 & $\mathbf{0}$ & $\mathbf{0}$ & 450 & 29.50 \\
\hline Geosat & 12.139 & 23.81 & 1554.37 & 79.78 & 3 & $\mathbf{0}$ & 635 & 64.40 \\
\hline Geosat & 12.139 & 36.18 & 1199.28 & 79.86 & 3 & $\mathbf{0}$ & 635 & 119.93 \\
\hline Fengyun 1B & 12.139 & 22.79 & 1795.75 & 129.68 & 1.8 & 1.4 & 881 & 81.07 \\
\hline Globalstar M023 & 12.139 & 35.56 & 2115.32 & 71.12 & 0 & 0 & 450 & 123.00 \\
\hline Globalstar M023 & 12.139 & 53.11 & 1694.06 & 71.18 & $\mathbf{0}$ & $\mathbf{0}$ & 450 & 83.62 \\
\hline Globalstar M023 & 12.139 & 24.49 & 2563.68 & 71.27 & $\mathbf{0}$ & $\mathbf{0}$ & 450 & 49.72 \\
\hline Globalstar M031 & 12.139 & 23.55 & 2702.65 & 8.85 & $\mathbf{0}$ & $\mathbf{0}$ & 450 & 141.75 \\
\hline Globalstar M031 & 12.139 & 47.45 & 1867.95 & 8.93 & $\mathbf{0}$ & $\mathbf{0}$ & 450 & 105.72 \\
\hline Globalstar M031 & 12.139 & 35.73 & 2181.59 & 9 & $\mathbf{0}$ & $\mathbf{0}$ & 450 & 65.59 \\
\hline Geosat & 12.139 & 26.04 & 1479.89 & 9.97 & 3 & $\mathbf{0}$ & 635 & 121.97 \\
\hline Globalstar M043 & 12.139 & 42.25 & 2687.51 & 11.2 & $\mathbf{0}$ & $\mathbf{0}$ & 450 & 100.47 \\
\hline Geosat & 12.139 & 55.50 & 915.83 & 33.34 & 3 & $\mathbf{0}$ & 635 & 75.04 \\
\hline Globalstar M043 & 12.139 & 36.88 & 2875.51 & 34.62 & $\mathbf{0}$ & $\mathbf{0}$ & 450 & 110.71 \\
\hline Globalstar M043 & 12.139 & 72.88 & 2113.58 & 34.73 & $\mathbf{0}$ & $\mathbf{0}$ & 450 & 49.66 \\
\hline Globalstar M031 & 12.139 & 22.27 & 2769.35 & 57 & $\mathbf{0}$ & $\mathbf{0}$ & 450 & 137.46 \\
\hline Globalstar M031 & 12.139 & 30.52 & 2375.07 & 57.07 & $\mathbf{0}$ & $\mathbf{0}$ & 450 & 108.71 \\
\hline Globalstar M031 & 12.139 & 22.60 & 2745.32 & 57.13 & $\mathbf{0}$ & $\mathbf{0}$ & 450 & 78.13 \\
\hline Cosmos 1900 & 12.139 & 51.43 & 920.28 & 14.36 & $\mathbf{0}$ & $\mathbf{0}$ & 1500 & 86.38 \\
\hline Globalstar M043 & 12.139 & 34.13 & 2985.84 & 58.05 & $\mathbf{0}$ & $\mathbf{0}$ & 450 & 116.79 \\
\hline Globalstar M043 & 12.139 & 65.18 & 2196.08 & 58.15 & $\mathbf{0}$ & $\mathbf{0}$ & 450 & 58.70 \\
\hline Globalstar M043 & 12.139 & 44.05 & 2628.25 & 58.22 & $\mathbf{0}$ & $\mathbf{0}$ & 450 & 27.43 \\
\hline Globalstar M055 & 12.139 & 22.05 & 2894.03 & 14.97 & $\mathbf{0}$ & $\mathbf{0}$ & 450 & 86.00 \\
\hline Globalstar M055 & 12.139 & 30.85 & 2478.26 & 15.17 & $\mathbf{0}$ & $\mathbf{0}$ & 450 & 48.90 \\
\hline Oscar 36 & 12.139 & 49.94 & 801.43 & 14.13 & 1.1 & 0.885 & 325 & 61.72 \\
\hline
\end{tabular}

Continued... 


\begin{tabular}{|c|c|c|c|c|c|c|c|c|}
\hline Satellite & FOV $\left(^{\prime}\right)$ & el $\left({ }^{\circ}\right)$ & $R(\mathbf{k m})$ & TLE(hr) & D1 & D2 & Mass(kg) & $\operatorname{SPA}\left({ }^{\circ}\right)$ \\
\hline IMS-1 & 12.139 & 41.80 & 906.44 & 9.91 & 0.6 & 0.6 & 83 & 55.65 \\
\hline Globalstar M043 & 12.139 & 24.38 & 3468.03 & 153.87 & $\mathbf{0}$ & 0 & 450 & 130.76 \\
\hline Globalstar M043 & 12.139 & 47.56 & 2533.54 & 153.87 & $\mathbf{0}$ & $\mathbf{0}$ & 450 & 107.19 \\
\hline Globalstar M043 & 12.139 & 60.62 & 2258.87 & 153.87 & $\mathbf{0}$ & $\mathbf{0}$ & 450 & 34.93 \\
\hline Globalstar M055 & 12.139 & 27.64 & 2621.26 & 17.22 & $\mathbf{0}$ & $\mathbf{0}$ & 450 & 89.88 \\
\hline Globalstar M053 & 12.139 & 34.87 & 2922.32 & 16.38 & $\mathbf{0}$ & $\mathbf{0}$ & 450 & 77.39 \\
\hline Globalstar M053 & 12.139 & 47.81 & 2501.90 & 16.55 & $\mathbf{0}$ & $\mathbf{0}$ & 450 & 53.67 \\
\hline Globalstar M053 & 12.139 & 40.17 & 2729.06 & 39.93 & $\mathbf{0}$ & $\mathbf{0}$ & 450 & 46.66 \\
\hline Globalstar M036 & 12.139 & 21.77 & 3196.36 & 17.96 & $\mathbf{0}$ & $\mathbf{0}$ & 450 & 75.46 \\
\hline Globalstar M008 & 12.139 & 27.01 & 2600.46 & 17.13 & $\mathbf{0}$ & $\mathbf{0}$ & 450 & 83.86 \\
\hline Globalstar M053 & 12.139 & 30.58 & 3115.92 & 42.22 & $\mathbf{0}$ & $\mathbf{0}$ & 450 & 88.49 \\
\hline Globalstar M036 & 12.139 & 22.06 & 3180.69 & 19.95 & $\mathbf{0}$ & $\mathbf{0}$ & 450 & 122.89 \\
\hline Globalstar M036 & 12.139 & 42.52 & 2311.93 & 20.06 & $\mathbf{0}$ & $\mathbf{0}$ & 450 & 79.66 \\
\hline Globalstar M036 & 12.139 & 36.12 & 2516.11 & 20.11 & $\mathbf{0}$ & $\mathbf{0}$ & 450 & 66.70 \\
\hline Globalstar M050 & 12.139 & 27.76 & 2741.75 & 19.7 & $\mathbf{0}$ & $\mathbf{0}$ & 450 & 71.84 \\
\hline Globalstar M036 & 12.139 & 32.59 & 2657.15 & 24.09 & $\mathbf{0}$ & $\mathbf{0}$ & 450 & 69.30 \\
\hline Globalstar M026 & 12.139 & 21.27 & 2722.63 & 24.58 & $\mathbf{0}$ & $\mathbf{0}$ & 450 & 62.10 \\
\hline Globalstar M026 & 12.139 & 24.23 & 2572.40 & 24.73 & $\mathbf{0}$ & $\mathbf{0}$ & 450 & 36.64 \\
\hline Globalstar M028 & 12.139 & 23.08 & 2623.85 & 16.44 & $\mathbf{0}$ & $\mathbf{0}$ & 450 & 73.65 \\
\hline Globalstar M028 & 12.139 & 36.88 & 2064.96 & 16.59 & $\mathbf{0}$ & $\mathbf{0}$ & 450 & 43.15 \\
\hline Globalstar M022 & 12.139 & 21.41 & 3110.48 & 17.66 & $\mathbf{0}$ & $\mathbf{0}$ & 450 & 85.20 \\
\hline Globalstar M026 & 12.139 & 25.60 & 2507.94 & 26.74 & $\mathbf{0}$ & $\mathbf{0}$ & 450 & 85.36 \\
\hline Globalstar M028 & 12.139 & 21.26 & 2733.71 & 18.63 & $\mathbf{0}$ & $\mathbf{0}$ & 450 & 93.35 \\
\hline Globalstar M052 & 12.139 & 21.09 & 2740.73 & 26.47 & $\mathbf{0}$ & $\mathbf{0}$ & 450 & 125.82 \\
\hline Globalstar M052 & 12.139 & 36.58 & 2075.33 & 26.52 & $\mathbf{0}$ & $\mathbf{0}$ & 450 & 110.14 \\
\hline Globalstar M052 & 12.139 & 57.32 & 1623.80 & 26.55 & $\mathbf{0}$ & $\mathbf{0}$ & 450 & 89.15 \\
\hline Globalstar M022 & 12.139 & 26.49 & 2834.07 & 19.76 & $\mathbf{0}$ & $\mathbf{0}$ & 450 & 96.16 \\
\hline Globalstar M022 & 12.139 & 20.23 & 3181.58 & 19.79 & $\mathbf{0}$ & $\mathbf{0}$ & 450 & 89.72 \\
\hline Globalstar M058 & 20.896 & 22.10 & 3132.79 & 20.46 & $\mathbf{0}$ & $\mathbf{0}$ & 450 & 142.91 \\
\hline Globalstar M058 & 20.896 & 63.02 & 1874.83 & 20.58 & $\mathbf{0}$ & $\mathbf{0}$ & 450 & 80.16 \\
\hline Globalstar M058 & 20.896 & 54.54 & 2002.75 & 20.61 & $\mathbf{0}$ & $\mathbf{0}$ & 450 & 55.06 \\
\hline Globalstar M058 & 20.896 & 38.21 & 2408.72 & 20.64 & $\mathbf{0}$ & $\mathbf{0}$ & 450 & 33.25 \\
\hline Globalstar M032 & 20.896 & 23.69 & 2601.69 & 7.9 & $\mathbf{0}$ & $\mathbf{0}$ & 450 & 132.76 \\
\hline Globalstar M032 & 20.896 & 39.82 & 1979.48 & 7.98 & $\mathbf{0}$ & $\mathbf{0}$ & 450 & 82.05 \\
\hline Globalstar M032 & 20.896 & 32.12 & 2228.53 & 8.03 & $\mathbf{0}$ & $\mathbf{0}$ & 450 & 57.45 \\
\hline Globalstar M008 & 20.896 & 22.76 & 2816.83 & 21.14 & $\mathbf{0}$ & $\mathbf{0}$ & 450 & 135.63 \\
\hline Globalstar M008 & 20.896 & 39.49 & 2128.62 & 21.28 & $\mathbf{0}$ & $\mathbf{0}$ & 450 & 62.80 \\
\hline Globalstar M008 & 20.896 & 27.09 & 2592.58 & 21.33 & $\mathbf{0}$ & $\mathbf{0}$ & 450 & 43.13 \\
\hline Globalstar M027 & 20.896 & 21.30 & 2724.70 & 16.58 & $\mathbf{0}$ & $\mathbf{0}$ & 450 & 103.59 \\
\hline Globalstar M027 & 20.896 & 42.61 & 1904.05 & 16.63 & $\mathbf{0}$ & $\mathbf{0}$ & 450 & 101.97 \\
\hline Globalstar M027 & 20.896 & 21.44 & 2722.84 & 16.78 & $\mathbf{0}$ & $\mathbf{0}$ & 450 & 93.04 \\
\hline Globalstar M058 & 20.896 & 22.01 & 3137.03 & 25.94 & $\mathbf{0}$ & $\mathbf{0}$ & 450 & 121.12 \\
\hline Globalstar M058 & 20.896 & 31.19 & 2674.81 & 25.99 & $\mathbf{0}$ & $\mathbf{0}$ & 450 & 106.93 \\
\hline Globalstar M058 & 20.896 & 41.59 & 2303.06 & 26.02 & $\mathbf{0}$ & $\mathbf{0}$ & 450 & 82.69 \\
\hline Globalstar M058 & 20.896 & 39.47 & 2365.88 & 26.09 & $\mathbf{0}$ & $\mathbf{0}$ & 450 & 52.81 \\
\hline Globalstar M032 & 20.896 & 24.23 & 2572.93 & 9.88 & $\mathbf{0}$ & $\mathbf{0}$ & 450 & 121.15 \\
\hline Globalstar M032 & 20.896 & 44.80 & 1852.04 & 9.93 & $\mathbf{0}$ & $\mathbf{0}$ & 450 & 99.28 \\
\hline Globalstar M032 & 20.896 & 62.31 & 1561.06 & 9.96 & $\mathbf{0}$ & $\mathbf{0}$ & 450 & 79.24 \\
\hline Globalstar M008 & 20.896 & 22.26 & 2842.02 & 23.18 & $\mathbf{0}$ & $\mathbf{0}$ & 450 & 119.63 \\
\hline Globalstar M008 & 20.896 & 40.01 & 2113.02 & 23.23 & $\mathbf{0}$ & $\mathbf{0}$ & 450 & 99.84 \\
\hline Globalstar M008 & 20.896 & 62.71 & 1673.59 & 23.28 & 0 & $\mathbf{0}$ & 450 & 69.16 \\
\hline
\end{tabular}


Table 7.3: Observational Results - Photometry Data Part 2 (Observational data relevant to photometric analysis-listed are the apparent magnitudes from each source, including the companion star).

\begin{tabular}{|c|c|c|c|c|c|}
\hline Satellite & Star & N2YO & Predicted Low & Predicted High & Actual \\
\hline Globalstar M049 & 7.82 & 7.70 & 7.13 & 9.64 & 8.73 \\
\hline Globalstar M003 & 7.62 & 8.18 & 7.77 & 10.27 & 9.06 \\
\hline Globalstar M002 & 5.23 & 8.20 & 7.01 & 9.52 & 4.93 \\
\hline Meteor 3-5 & 6.42 & 7.68 & 2.56 & 6.51 & 6.54 \\
\hline Globalstar M003 & 7.51 & 8.04 & 6.99 & 9.49 & 10.07 \\
\hline Fengyun 1B & 7.69 & 6.98 & 7.15 & 10.19 & 7.64 \\
\hline Fengyun 1B & 8.16 & 7.31 & 8.75 & 11.80 & 9.60 \\
\hline Geosat & 6.30 & 6.13 & 6.30 & 8.80 & 7.59 \\
\hline Globalstar M051 & 8.14 & 7.65 & 6.67 & 9.17 & 9.82 \\
\hline Globalstar M003 & 7.31 & 8.03 & 7.31 & 9.81 & 8.80 \\
\hline Geosat & 7.63 & 6.72 & 5.23 & 7.73 & 6.38 \\
\hline Globalstar M051 & 8.19 & 7.95 & 6.87 & 9.37 & 9.46 \\
\hline Globalstar M003 & 7.07 & 8.70 & 10.65 & 13.15 & 9.42 \\
\hline Globalstar M003 & 8.12 & 7.88 & 8.08 & 10.58 & 8.26 \\
\hline Globalstar M003 & 6.14 & 8.58 & 7.37 & 9.87 & 8.88 \\
\hline Fengyun 1B & 5.69 & 7.74 & 7.48 & 10.52 & 7.97 \\
\hline Fengyun 1B & 5.77 & 6.83 & 8.11 & 11.15 & 8.92 \\
\hline Globalstar M023 & 7.23 & 7.58 & 8.30 & 10.80 & 9.70 \\
\hline Geosat & 8.07 & 6.72 & 6.87 & 9.37 & 7.51 \\
\hline Globalstar M003 & 8.25 & 7.96 & 7.78 & 10.29 & 10.58 \\
\hline Globalstar M023 & 6.22 & 7.33 & 5.60 & 8.10 & 7.96 \\
\hline Geosat & 6.63 & 6.82 & 5.48 & 7.98 & 6.54 \\
\hline Geosat & 7.77 & 6.08 & 6.57 & 9.07 & 7.45 \\
\hline Fengyun 1B & 7.51 & 7.63 & 7.29 & 10.34 & 8.65 \\
\hline Globalstar M023 & 7.01 & 7.25 & 8.00 & 10.50 & 8.96 \\
\hline Globalstar M023 & 7.13 & 6.62 & 5.94 & 8.44 & 8.38 \\
\hline Globalstar M023 & 6.51 & 7.84 & 6.34 & 8.85 & 7.92 \\
\hline Globalstar M031 & 7.10 & 8.47 & 9.90 & 12.40 & 8.58 \\
\hline Globalstar M031 & 6.83 & 7.35 & 6.89 & 9.39 & 8.89 \\
\hline Globalstar M031 & 7.66 & 7.80 & 6.15 & 8.65 & 7.97 \\
\hline Geosat & 8.07 & 6.77 & 7.23 & 9.73 & 7.80 \\
\hline Globalstar M043 & 7.16 & 8.28 & 7.50 & 10.00 & 7.24 \\
\hline Geosat & 7.76 & 5.36 & 4.34 & 6.84 & 5.07 \\
\hline Globalstar M043 & 6.84 & 8.45 & 8.08 & 10.58 & 6.51 \\
\hline Globalstar M043 & 6.86 & 7.63 & 5.69 & 8.20 & 7.13 \\
\hline Globalstar M031 & 6.08 & 8.46 & 9.64 & 12.14 & 8.73 \\
\hline Globalstar M031 & 8.14 & 7.93 & 7.63 & 10.13 & 9.83 \\
\hline Globalstar M031 & 7.81 & 8.44 & 7.08 & 9.58 & 9.23 \\
\hline Cosmos 1900 & 8.21 & 5.93 & 4.05 & 6.55 & 5.29 \\
\hline Globalstar M043 & 7.51 & 8.57 & 8.45 & 10.95 & 8.04 \\
\hline Globalstar M043 & 6.52 & 7.71 & 5.93 & 8.43 & 7.16 \\
\hline Globalstar M043 & 8.43 & 8.22 & 5.98 & 8.48 & 7.13 \\
\hline Globalstar M055 & 7.64 & 8.18 & 7.41 & 9.91 & 6.01 \\
\hline Globalstar M055 & 7.68 & 7.69 & 6.18 & 8.68 & 7.76 \\
\hline Oscar 36 & 8.17 & 8.58 & 5.97 & 8.95 & 7.72 \\
\hline IMS-1 & 8.37 & 9.35 & 7.48 & 9.98 & 7.55 \\
\hline Globalstar M043 & 8.28 & 8.96 & 9.64 & 12.14 & 9.90 \\
\hline Globalstar M043 & 8.06 & 8.13 & 7.61 & 10.11 & 8.01 \\
\hline Globalstar M043 & 7.12 & 7.85 & 5.67 & 8.17 & 7.58 \\
\hline Globalstar M055 & 7.90 & 7.78 & 7.22 & 9.72 & 7.82 \\
\hline Globalstar M053 & 7.73 & 8.49 & 7.05 & 9.55 & 10.74 \\
\hline Globalstar M053 & 6.82 & 8.07 & 6.17 & 8.67 & 7.08 \\
\hline Globalstar M053 & 7.77 & 8.33 & 6.29 & 8.79 & 8.23 \\
\hline Globalstar M036 & 8.79 & 7.78 & 7.36 & 9.86 & 10.89 \\
\hline Globalstar M008 & 8.39 & 8.34 & 7.04 & 9.54 & 8.35 \\
\hline Globalstar M053 & 7.35 & 8.73 & 7.52 & 10.02 & 9.88 \\
\hline Globalstar M036 & 8.43 & 7.85 & 9.04 & 11.54 & 11.22 \\
\hline
\end{tabular}




\begin{tabular}{|c|c|c|c|c|c|}
\hline Satellite & Star & N2YO & Predicted Low & Predicted High & Actual \\
\hline Globalstar M036 & 8.51 & 6.82 & 6.55 & 9.05 & 9.08 \\
\hline Globalstar M036 & 7.81 & 7.08 & 6.48 & 8.98 & 5.60 \\
\hline Globalstar M050 & 5.33 & 8.24 & 6.85 & 9.35 & 8.66 \\
\hline Globalstar M036 & 8.02 & 7.21 & 6.68 & 9.18 & 6.87 \\
\hline Globalstar M026 & 7.07 & 7.99 & 6.74 & 9.24 & 10.07 \\
\hline Globalstar M026 & 8.51 & 7.78 & 6.19 & 8.69 & 8.19 \\
\hline Globalstar M028 & 6.03 & 8.48 & 6.86 & 9.36 & 8.31 \\
\hline Globalstar M028 & 8.47 & 7.74 & 5.66 & 8.16 & 6.78 \\
\hline Globalstar M022 & 7.32 & 8.27 & 7.55 & 10.05 & 9.95 \\
\hline Globalstar M026 & 7.05 & 7.76 & 7.02 & 9.52 & 9.00 \\
\hline Globalstar M028 & 8.47 & 8.59 & 7.52 & 10.02 & 9.25 \\
\hline Globalstar M052 & 8.16 & 8.03 & 8.90 & 11.40 & 8.59 \\
\hline Globalstar M052 & 8.06 & 7.25 & 7.35 & 9.85 & 8.46 \\
\hline Globalstar M052 & 7.36 & 6.63 & 5.99 & 8.49 & 7.07 \\
\hline Globalstar M022 & 8.66 & 7.81 & 7.60 & 10.10 & 9.39 \\
\hline Globalstar M022 & 7.18 & 8.25 & 7.76 & 10.26 & 9.79 \\
\hline Globalstar M058 & 7.75 & 8.38 & 10.35 & 12.85 & 10.44 \\
\hline Globalstar M058 & 8.47 & 6.90 & 6.05 & 8.55 & 9.19 \\
\hline Globalstar M058 & 7.20 & 7.08 & 5.69 & 8.19 & 7.96 \\
\hline Globalstar M058 & 8.40 & 7.59 & 5.87 & 8.37 & 8.54 \\
\hline Globalstar M032 & 8.71 & 7.88 & 9.16 & 11.66 & 9.33 \\
\hline Globalstar M032 & 7.13 & 7.01 & 6.29 & 8.79 & 8.61 \\
\hline Globalstar M032 & 8.62 & 7.37 & 6.07 & 8.57 & 8.37 \\
\hline Globalstar M008 & 9.22 & 8.56 & 9.54 & 12.04 & 9.59 \\
\hline Globalstar M008 & 7.79 & 7.68 & 6.02 & 8.52 & 7.35 \\
\hline Globalstar M008 & 8.36 & 8.29 & 6.24 & 8.74 & 7.74 \\
\hline Globalstar M027 & 8.02 & 8.57 & 7.86 & 10.36 & 9.87 \\
\hline Globalstar M027 & 5.49 & 7.49 & 6.81 & 9.31 & 8.36 \\
\hline Globalstar M027 & 8.09 & 8.56 & 7.49 & 9.99 & 9.93 \\
\hline Globalstar M058 & 8.12 & 8.36 & 8.92 & 11.42 & 9.93 \\
\hline Globalstar M058 & 7.38 & 7.82 & 7.81 & 10.31 & 10.54 \\
\hline Globalstar M058 & 7.70 & 7.35 & 6.63 & 9.13 & 8.62 \\
\hline Globalstar M058 & 8.55 & 7.44 & 6.08 & 8.58 & 9.62 \\
\hline Globalstar M032 & 8.29 & 7.82 & 8.45 & 10.95 & 8.97 \\
\hline Globalstar M032 & 8.68 & 6.91 & 6.64 & 9.14 & 7.90 \\
\hline Globalstar M032 & 8.44 & 6.47 & 5.63 & 8.13 & 7.27 \\
\hline Globalstar M008 & 6.79 & 8.65 & 8.62 & 11.12 & 9.30 \\
\hline Globalstar M008 & 6.93 & 7.79 & 6.97 & 9.47 & 7.92 \\
\hline Globalstar M008 & 8.35 & 7.17 & 5.55 & 8.05 & 5.27 \\
\hline
\end{tabular}

Table 7.4: Additional Single Pass Orbit Determination Results (Comparison of COE's generated by angles-only orbit determination method, to COE's gathered from propagation-source TLE's).

\begin{tabular}{|c|c|c|c|c|c|c|c|}
\hline Satellite & Source & Epoch (UT) & A (km) & $e$ & $i\left({ }^{\circ}\right)$ & $\Omega\left({ }^{\circ}\right)$ & $\omega\left(^{\circ}\right)$ \\
\hline \multirow{4}{*}{$\begin{array}{c}\text { Globalstar } \\
\text { M023 }\end{array}$} & TLE & $3 / 26 \quad 19: 58$ & $\begin{array}{l}7792.7 \\
\end{array}$ & $1.09 \mathrm{E}-3$ & 52.01 & 327.39 & 356.78 \\
\hline & Vallado Gauss & \multirow{3}{*}{ 3/27 4:07 } & 7621.9 & 2.40E-2 & 52.01 & 326.20 & 323.70 \\
\hline & Curtis Gauss & & 7622.4 & 2.12E-2 & 52.09 & 326.25 & 323.66 \\
\hline & Double-R & & 7622.4 & 2.12E-2 & 52.09 & 326.25 & 323.66 \\
\hline \multirow{4}{*}{$\begin{array}{c}\text { Globalstar } \\
\text { M031 } \\
\end{array}$} & TLE & 4/12 18:41 & 7855.9 & $1.17 \mathrm{E}-4$ & 52.00 & 333.63 & 97.06 \\
\hline & Vallado Gauss & \multirow{3}{*}{ 4/12 3:49 } & 8204.1 & 3.72E-2 & 51.92 & 335.06 & 140.32 \\
\hline & Curtis Gauss & & 8194.5 & $3.88 \mathrm{E}-2$ & 52.01 & 335.13 & 139.15 \\
\hline & Double-R & & 8194.5 & $3.88 \mathrm{E}-2$ & 52.01 & 335.13 & 139.15 \\
\hline \multirow{4}{*}{$\begin{array}{c}\text { Globalstar } \\
\text { M043 }\end{array}$} & TLE & 4/16 $3: 58$ & 8412.5 & 8.40E-4 & 51.94 & 17.10 & 102.54 \\
\hline & Vallado Gauss & \multirow{3}{*}{ 4/14 4:57 } & 8648.9 & $2.08 \mathrm{E}-2$ & 52.02 & 21.78 & 123.18 \\
\hline & Curtis Gauss & & 8585.1 & $1.83 \mathrm{E}-2$ & 51.97 & 21.68 & 124.99 \\
\hline & Double-R & & 8585.1 & $1.83 \mathrm{E}-2$ & 51.97 & 21.68 & 124.99 \\
\hline \multicolumn{2}{|r|}{ tar M043 } & 4/17 3:58 & \multicolumn{5}{|c|}{ Did Not Converge } \\
\hline
\end{tabular}




\subsection{Lessons Learned}

\section{Anticipate Bad Weather}

Anticipating the disruption of an observation schedule due to poor weather is a common sense issue, but it can not be overstated the extent to which a few cloudy nights can severely reduce the quantity of data acquired. In the case of this project, 4 out of the last 5 planned nights of observations were canceled due to weather. Considering that 23 of the 96 successful observations were recorded on the final observation night, both the quantity and diversity of satellites observed would have increased dramatically had the final nights of observations not been canceled. It is advised that observations should be planned for seasons of the year with the lowest level of cloudy weather, and telescope operators should be flexible concerning the nights on which they observe.

\section{Simple Methods Get Results}

Throughout this project, much time and resources were devoted to pursuing observation techniques that were more powerful or efficient than the simple, staring method used for the entirety of the thesis. At different points in time, both active-tracking and a scope fence were considered. Tools were developed to determine the pointing operations of multiple telescopes involved in a scope fence, and active-tracking controllers such as Sidereal Technology's SiTech controller were researched. The staring method was only attempted once the other, more complicated methods were met with operational difficulties. If the staring method had been introduced earlier in the year, the likely outcome would have been an increase in total data acquired. 


\section{Current-J2000 Epoch Mistake}

In the first weeks of observations, before the installation of the $\mathrm{f} / 3.3$ focal reducer, the success rate of capturing satellites was very low-only 2 of 29 observations caught the target satellite. At first, it was thought this low success rate was due to either an inaccuracy in the TLE propagation-leading to cross-track errors larger than the FOV of the telescope - or the satellites were too dim to be observed. Much effort was put into rectifying this supposed problem of an insufficient FOV, including selecting satellite candidates for young TLE ages $(<1$ day) and low elevation observations (to reduce the angular cross track error). In addition to the clear option of adding a focal reducer to the system, an optical fence (multiple telescopes used in unison) was also considered. Once a focal reducer was acquired and put into place, the success rate improved greatly but remained lower than expected: 75 of 133 observations were successful. Attempts to explain and correct this shortfall continued: recording time was increased from $\pm 10 \mathrm{~s}$, to $\pm 30 \mathrm{~s}$, to ensure along-track error was not a problem, and analysis was completed to demonstrate that observation failures were not exclusive to low-magnitude satellites.

It was not until late April (the third month of observations) that the main cause of the issue was uncovered. When RA/Dec angles were generated by MATLAB and STK, they were produced in the Current Epoch frame of reference. As the Earth's spin axis slowly precesses over a $\sim 26,000$ year cycle, the vernal equinox, pictured in Fig. 2.2, gradually shifts. This shift alters the frame of reference in which the RA/Dec angles are measured. When RA/Dec target angles were being placed into SkyX, they were unknowingly designated as in the J2000 frame, not the Current 2013 frame. Depending on the declination of an angle set, the mismatch in the J2000 and Current frames resulted in errors between $\sim 1-10$ 
arcminutes. These errors can be seen in Appendix 7.1, wherein Figs. 7.1 through 7.3 were obtained before the epoch frame correction, and Fig. 7.4 was obtained after. Once this problem was identified, the correct conversion from Current to J2000 frame,

$$
\begin{array}{r}
\alpha_{J 2000}=\alpha_{\text {Current }}-Y S\left(0.01281467+0.0055645 \cos \left(\alpha_{\text {Current }}\right) \tan \left(\delta_{\text {Current }}\right)\right) \\
\delta_{J 2000}=\delta_{\text {Current }}-0.0055645 \times Y S \cos \left(\alpha_{\text {Current }}\right)
\end{array}
$$

where $Y S$ is years-since-2000, was applied to every future observation. After making this correction, 20 out of 20 observations were successful. For data taken before this issue was corrected, all angle-sets were retroactively converted to the J2000 frame in pixel_analysis.m.

\subsection{Custom List of Satellite Targets}




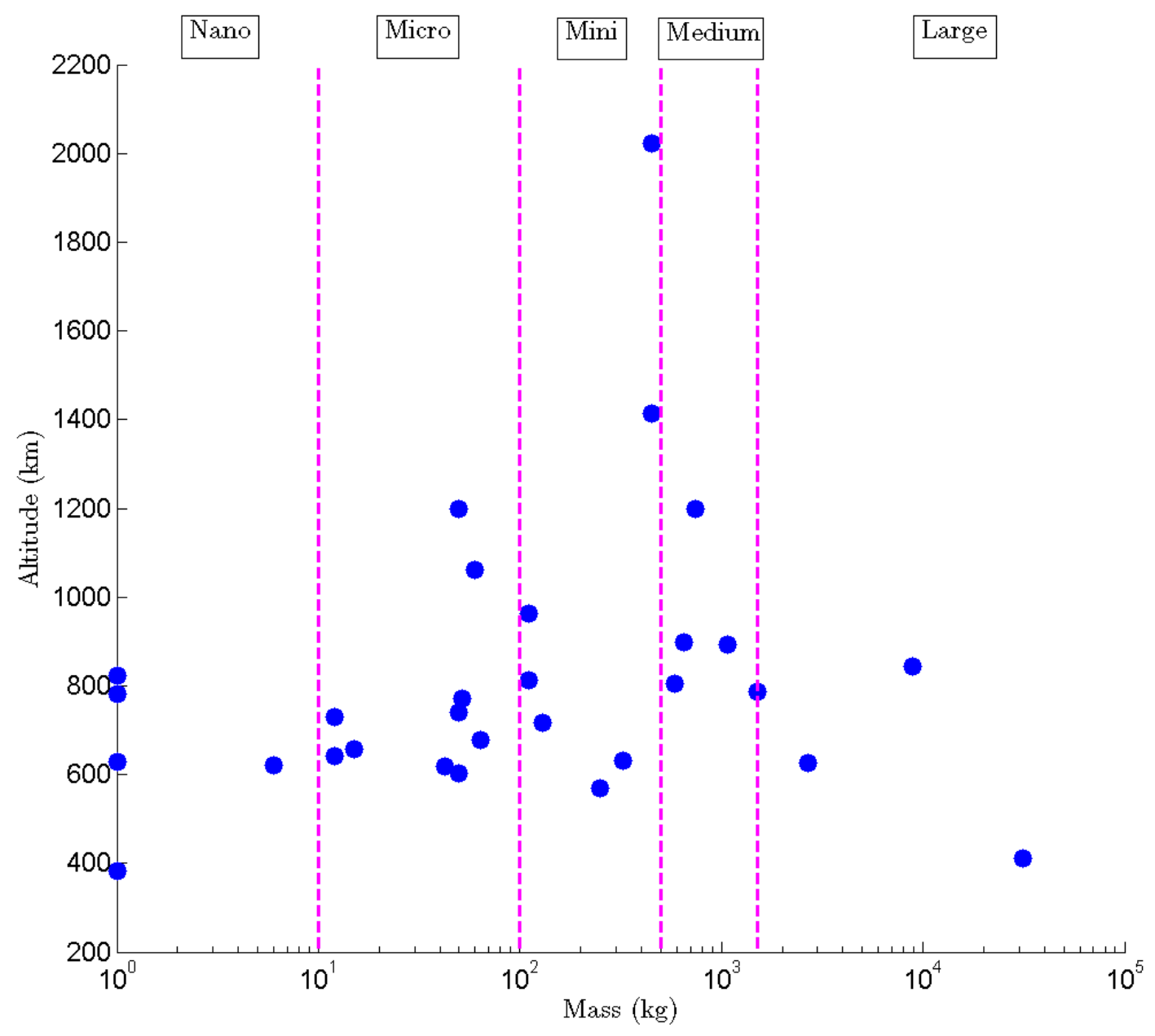

Figure 7.5: Altitude vs. Mass for Satellites of Interest List. 
Table 7.5: Satellites of Interest List.

\begin{tabular}{|c|c|c|c|c|c|}
\hline NORAD ID & Satellite & Period (min) & $i\left(^{\circ}\right)$ & Apogee $(\mathrm{km})$ & Perigee $(\mathrm{km})$ \\
\hline 2965 & OPS 4947 & 106.46 & 89.25 & 1100 & 1025 \\
\hline 13777 & IRAS & 102.84 & 99 & 908 & 879 \\
\hline 18187 & Cosmos 1867 & 100.63 & 65.01 & 803 & 774 \\
\hline 21263 & NOAA 12 & 101.01 & 98.7 & 817 & 796 \\
\hline 23088 & SL-16 R/B & 101.8 & 71 & 845 & 843 \\
\hline 23606 & Cerise & 96.74 & 98.34 & 606 & 599 \\
\hline 24954 & Faisat $2 \mathrm{~V}$ & 104.36 & 82.92 & 993 & 937 \\
\hline 25544 & ISS (ZARYA) & 92.87 & 51.65 & 421 & 409 \\
\hline 25621 & Globalstar M023 & 114.08 & 52.01 & 1421 & 1406 \\
\hline 25693 & Oscar 36 & 97.33 & 64.56 & 645 & 617 \\
\hline 25736 & Mublcom & 99.58 & 97.68 & 743 & 734 \\
\hline 25886 & Globalstar M053 & 127.7 & 52 & 2025 & 2019 \\
\hline 26064 & Falconsat & 100.3 & 100.19 & 800 & 745 \\
\hline 26702 & Odin & 96.03 & 97.64 & 571 & 565 \\
\hline 27608 & Unisat 2 & 97.58 & 64.56 & 659 & 626 \\
\hline 27848 & Cubesat XI-IV & 101.34 & 98.71 & 829 & 815 \\
\hline 27939 & Mozhayets 4 & 98.33 & 97.8 & 689 & 669 \\
\hline 28372 & Aprizesat 1 & 99.4 & 98.05 & 763 & 696 \\
\hline 28493 & Nanosat(1) & 97.85 & 98.38 & 658 & 653 \\
\hline 28650 & Hamsat & 97.04 & 97.57 & 635 & 599 \\
\hline 29678 & Corot & 102.98 & 90.01 & 904 & 895 \\
\hline 29712 & Pehuensat 1 & 96.72 & 97.65 & 611 & 592 \\
\hline 32786 & IMS-1 & 97.25 & 97.75 & 635 & 619 \\
\hline 36037 & Proba 2 & 99.15 & 98.3 & 728 & 707 \\
\hline 36122 & Xiwang-1 & 109.4 & 100.3 & 1204 & 1193 \\
\hline 36588 & Servis 2 & 109.44 & 100.43 & 1214 & 1188 \\
\hline 36797 & Aissat 1 & $\mathbf{9 7 . 1 4}$ & 98.03 & 631 & 613 \\
\hline 36834 & Yaogan 10 & 97.22 & 97.85 & 627 & 624 \\
\hline 38711 & MKA-FKI 1 & 101.14 & 98.94 & 822 & 804 \\
\hline 38853 & Fitsat 1 & 87.84 & 51.63 & 175 & 160 \\
\hline 39087 & AAUSAT3 & 100.41 & 98.63 & 787 & 770 \\
\hline 38763 & CP5 & 97.24 & 64.67 & 773 & 480 \\
\hline
\end{tabular}

\subsection{Explanation of Selected MATLAB Codes}

This appendix provides additional details for select MATLAB algorithms developed for this project - although only excerpts of each code are presented here, accompanied by commentary, the full files (along with algorithms not listed here) can be made available by contacting the author at bschmalzel@gmail.com.

angledet.m

This code determines the right ascension and declination values from images of a successful observation - the pixel locations for the companion star and two locations of the satellite, are provided as inputs. 


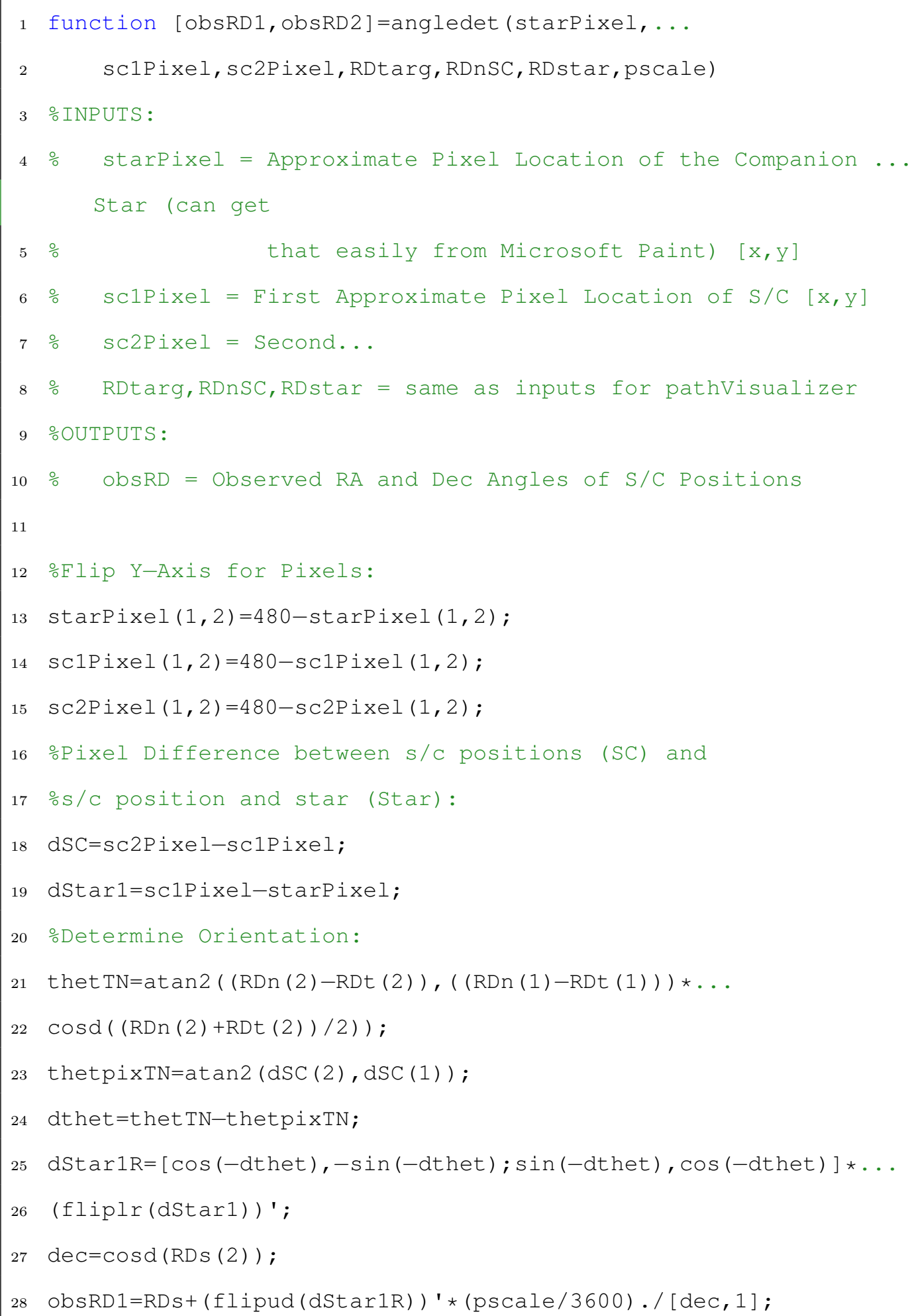


appMag.m

This function is used to estimate the apparent magnitude of a satellite, given a number of variables or assumptions concerning the satellite and its orbit.

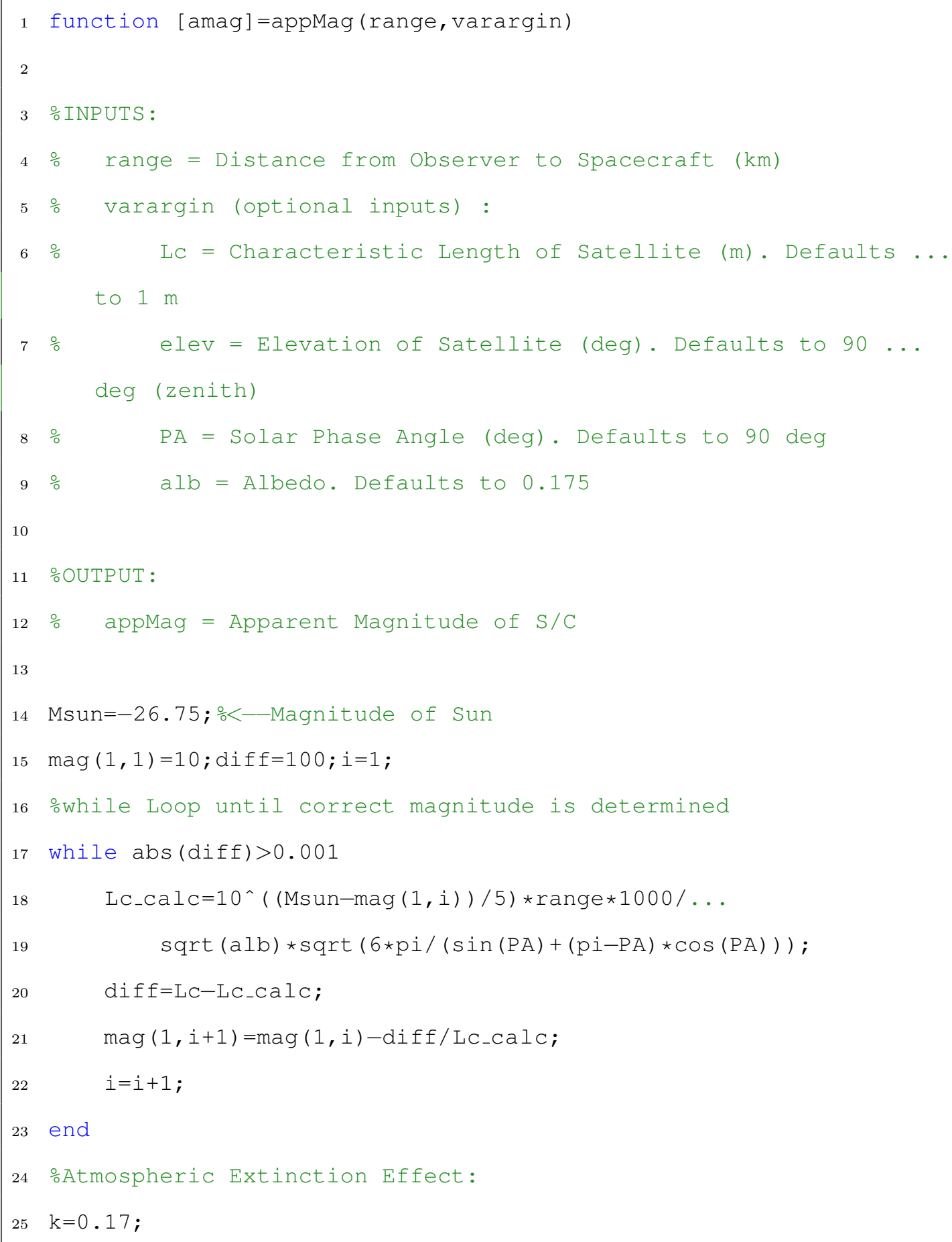




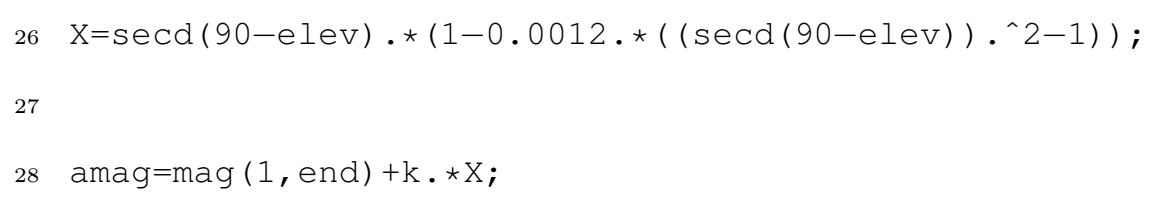

\section{imgPHOT.m}

This file is used to quantify the apparent brightness of an observed satellite. It does so through comparative photometry with the use of the companion, reference star within each image. Because the file is written to read-in specifically-named files, it would likely need to be edited for use in other projects.

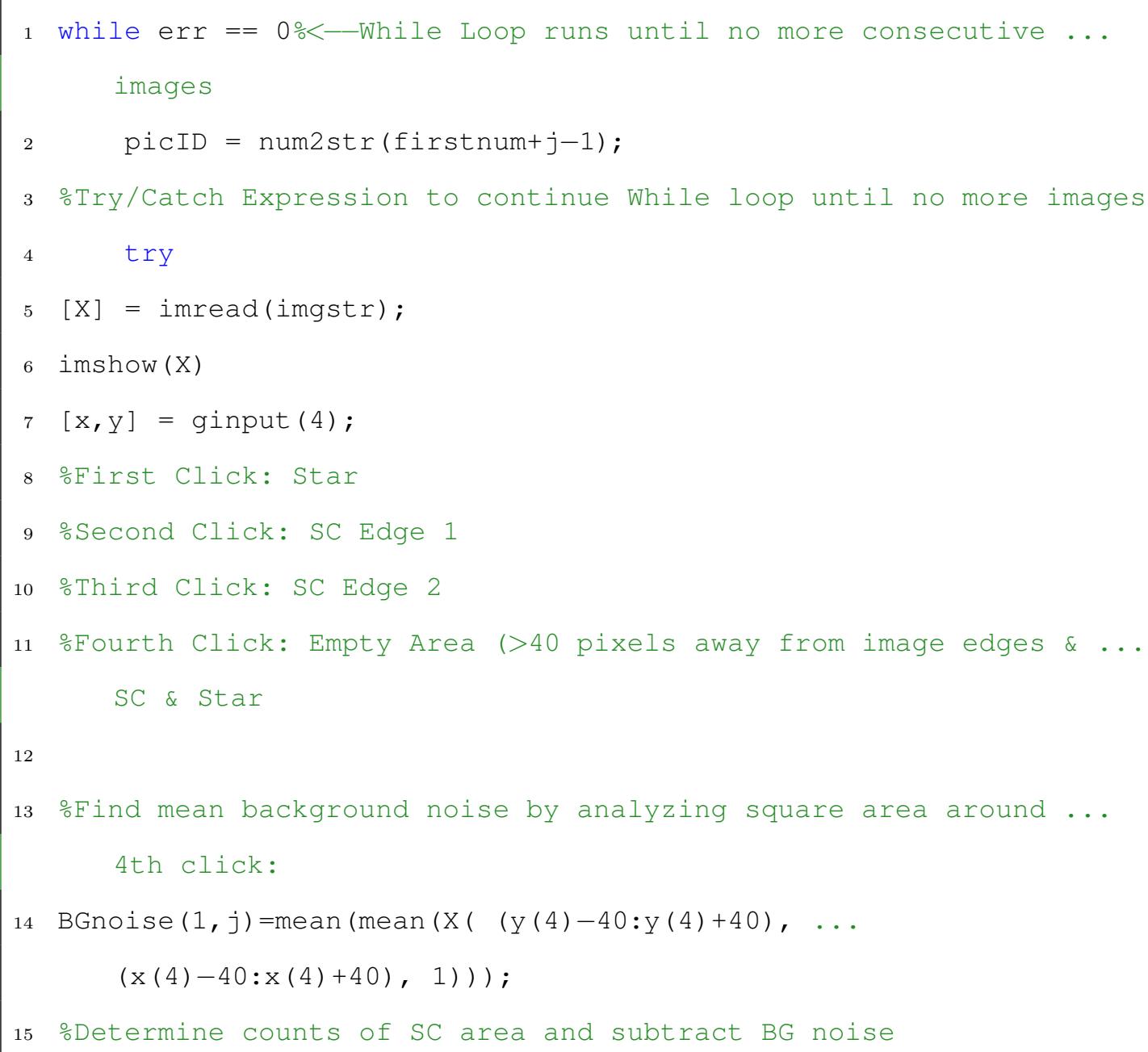




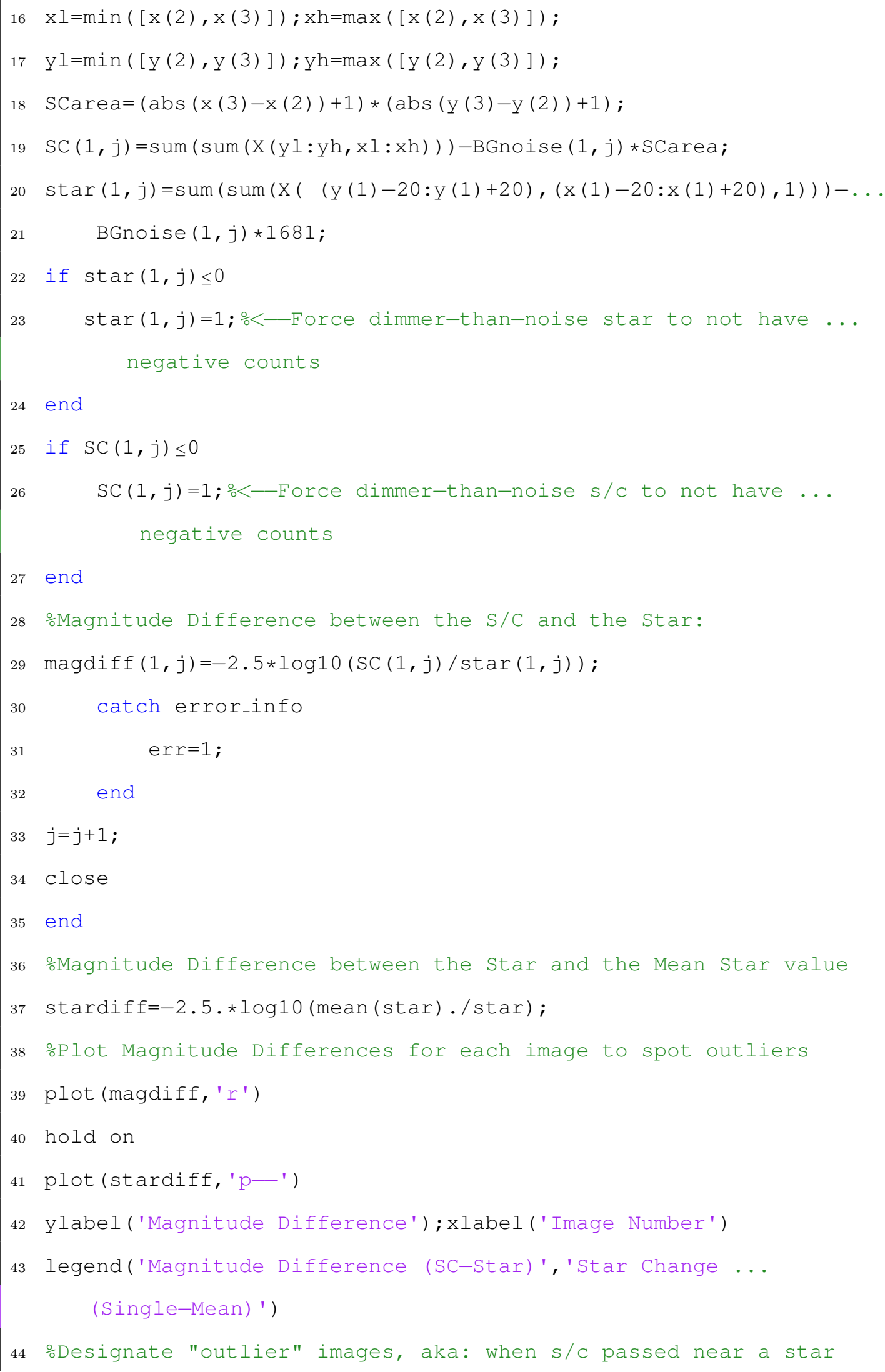




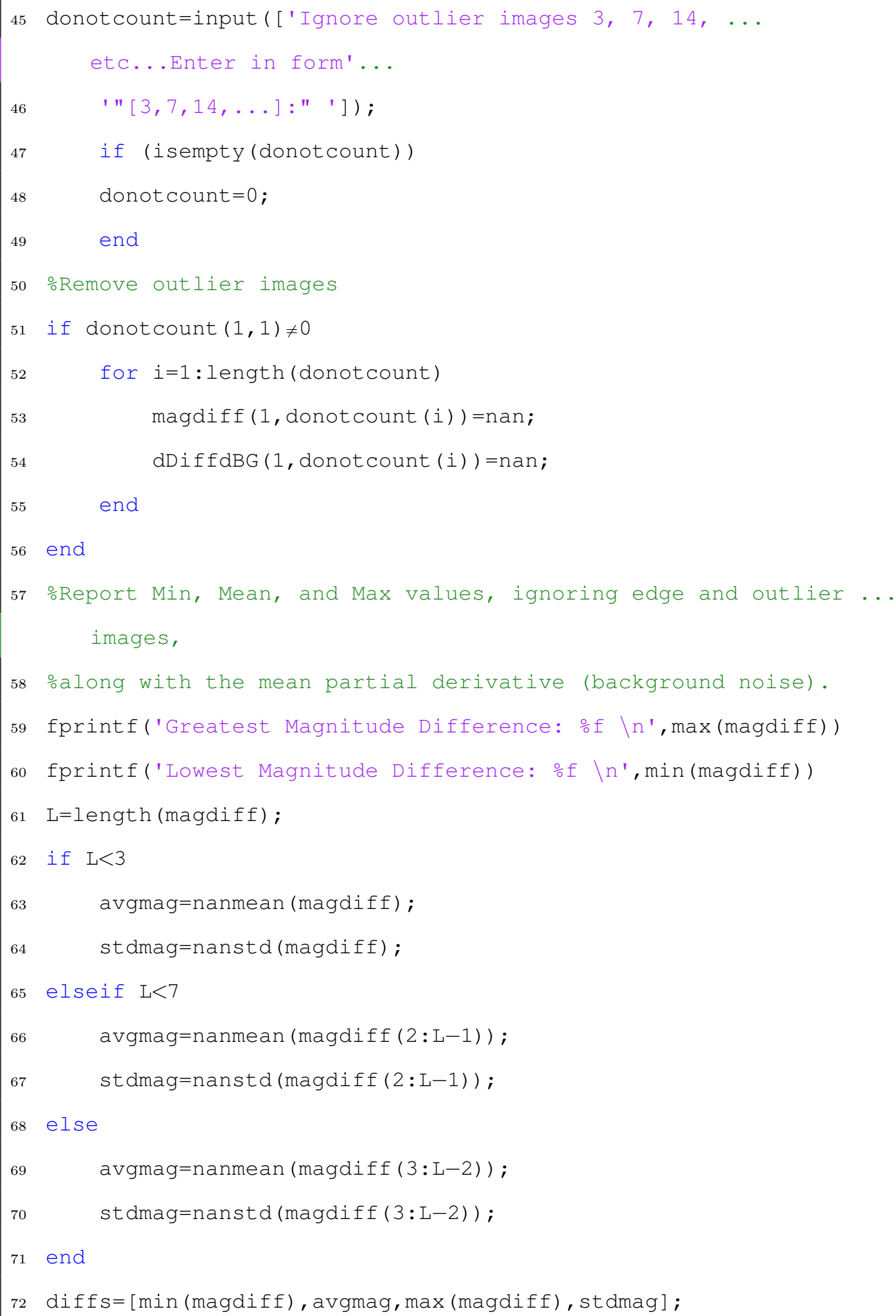




\section{ObservationResults.m}

This code inputs observation data from observations.xls, to be used in both data reduction and analysis, concerning magnitude prediction model validation.

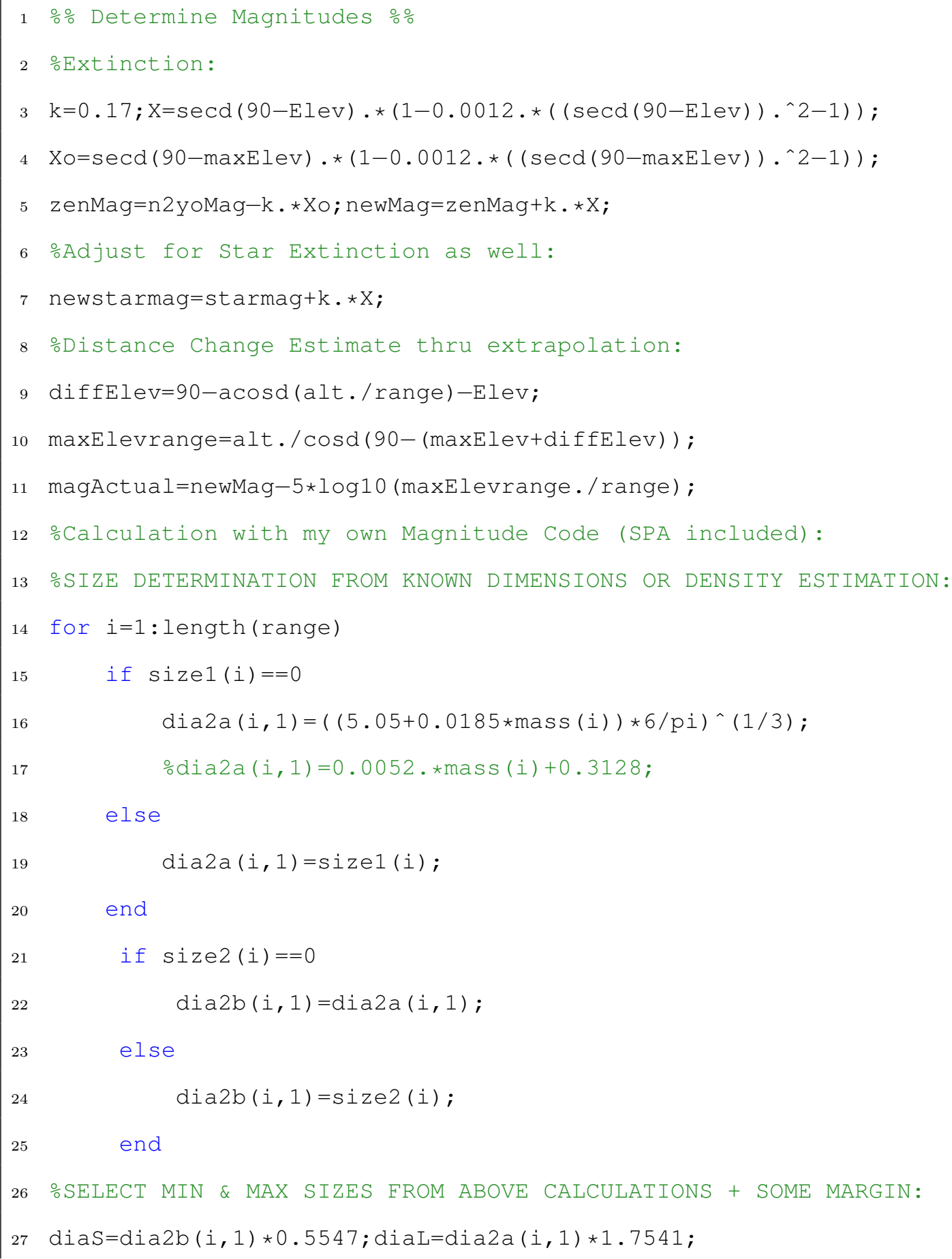




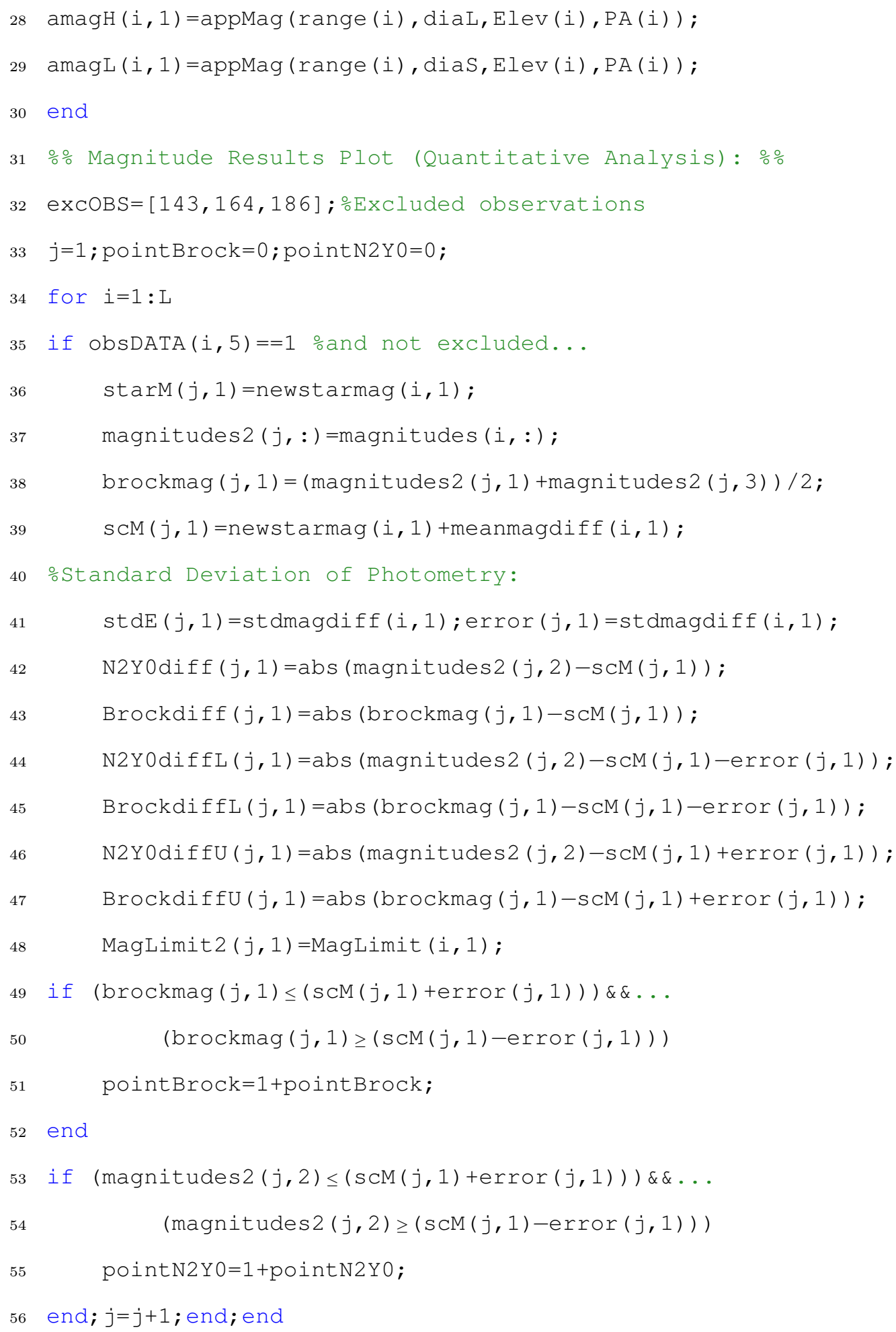


orbit_determination.m

This function is used to generate COE's given three observations of a satellite. The user has the ability to comment in/out the desired angles-only orbit determination technique.

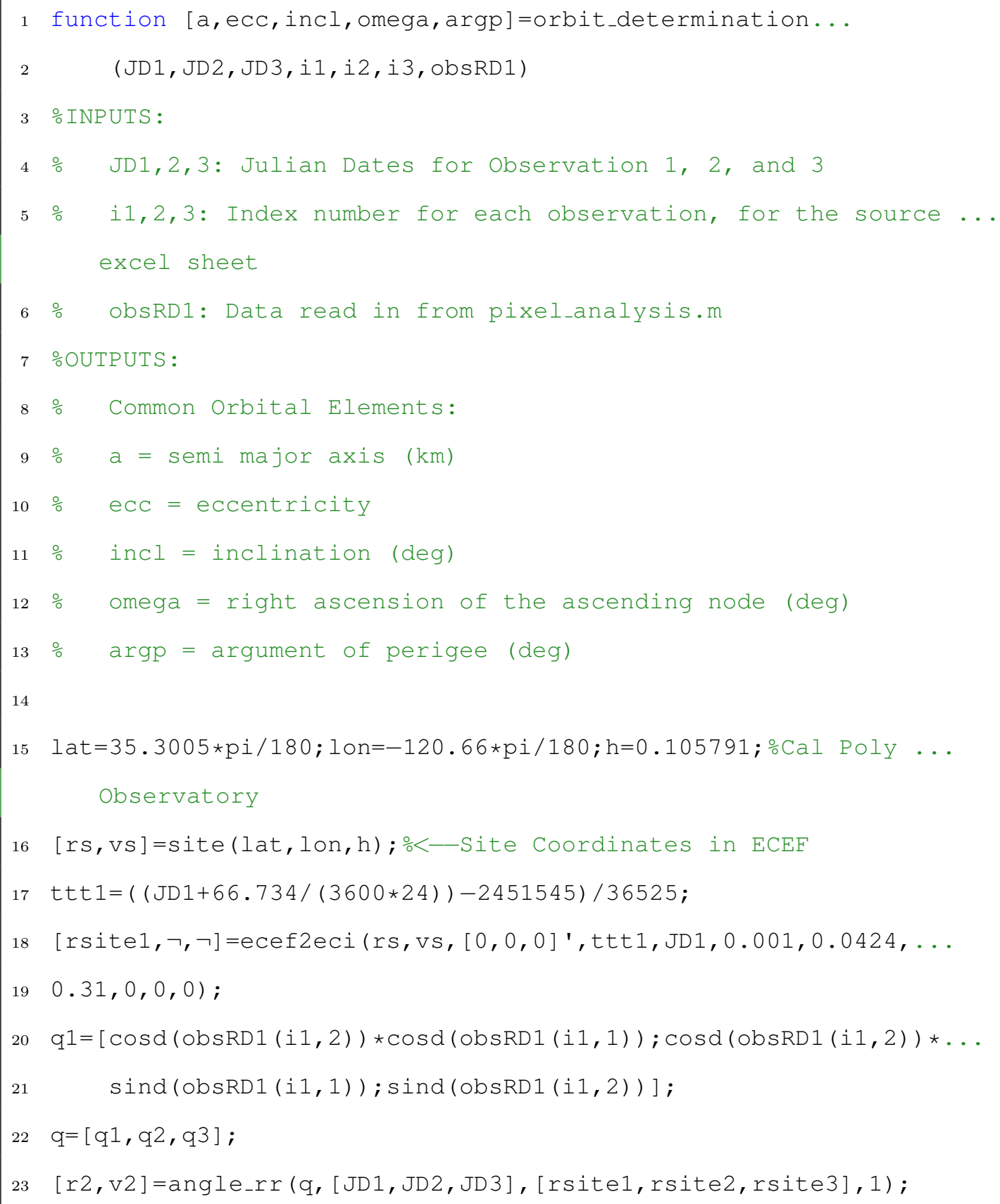


$24 \quad \mathrm{r} 2=\mathrm{r} 2{ }^{\prime} ; \mathrm{v} 2=\mathrm{v} 2^{\prime} ;$

$25[\mathrm{a}$, ecc, incl, omega, argp, $\neg]=\operatorname{RV} 2 \operatorname{CoE} 2(r 2, \mathrm{v} 2,4)$;

26 end

\section{passPredict.m}

This code predicts which satellites (from a Space Track favorites list) will be visible on a given night.

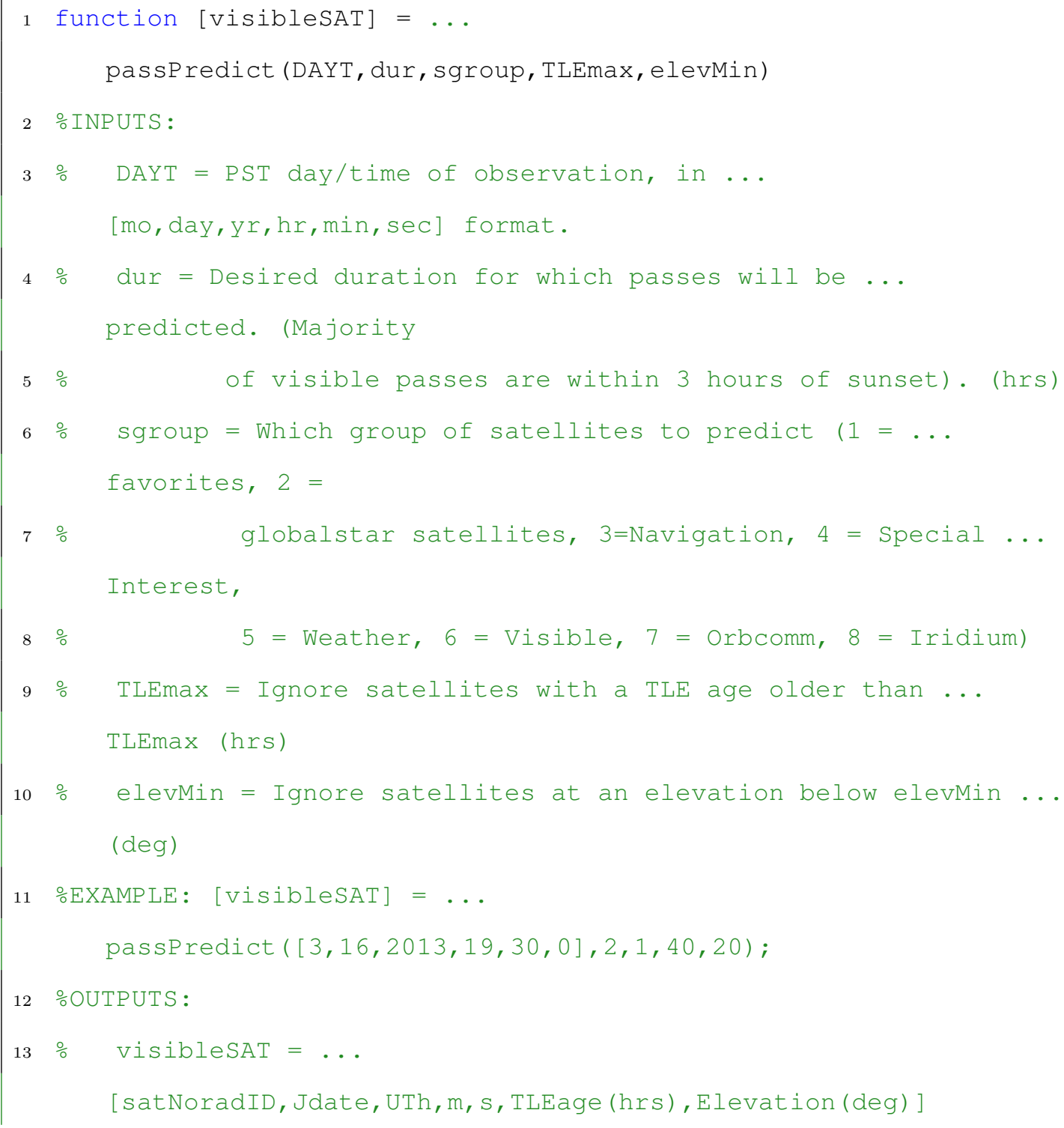




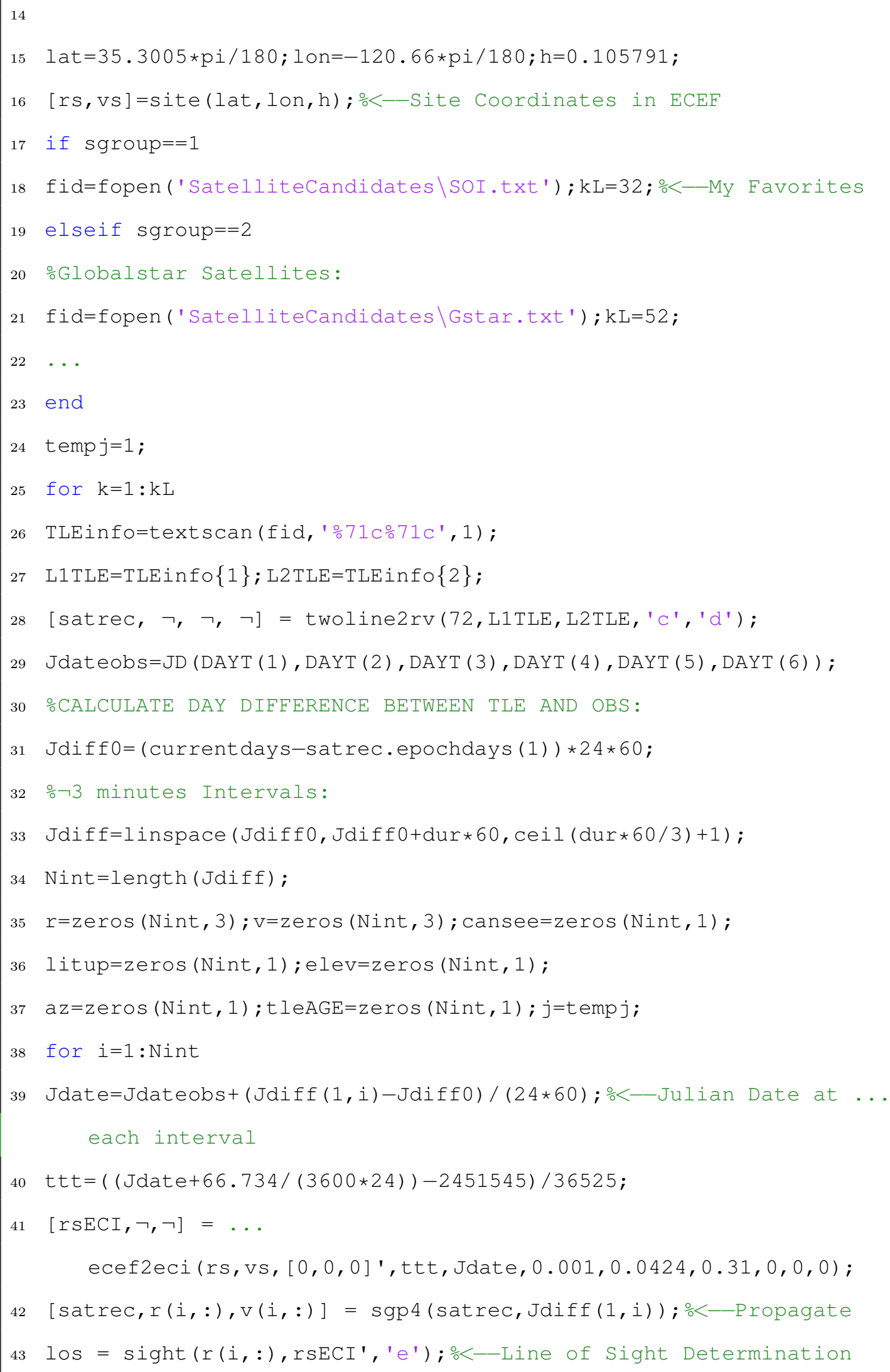




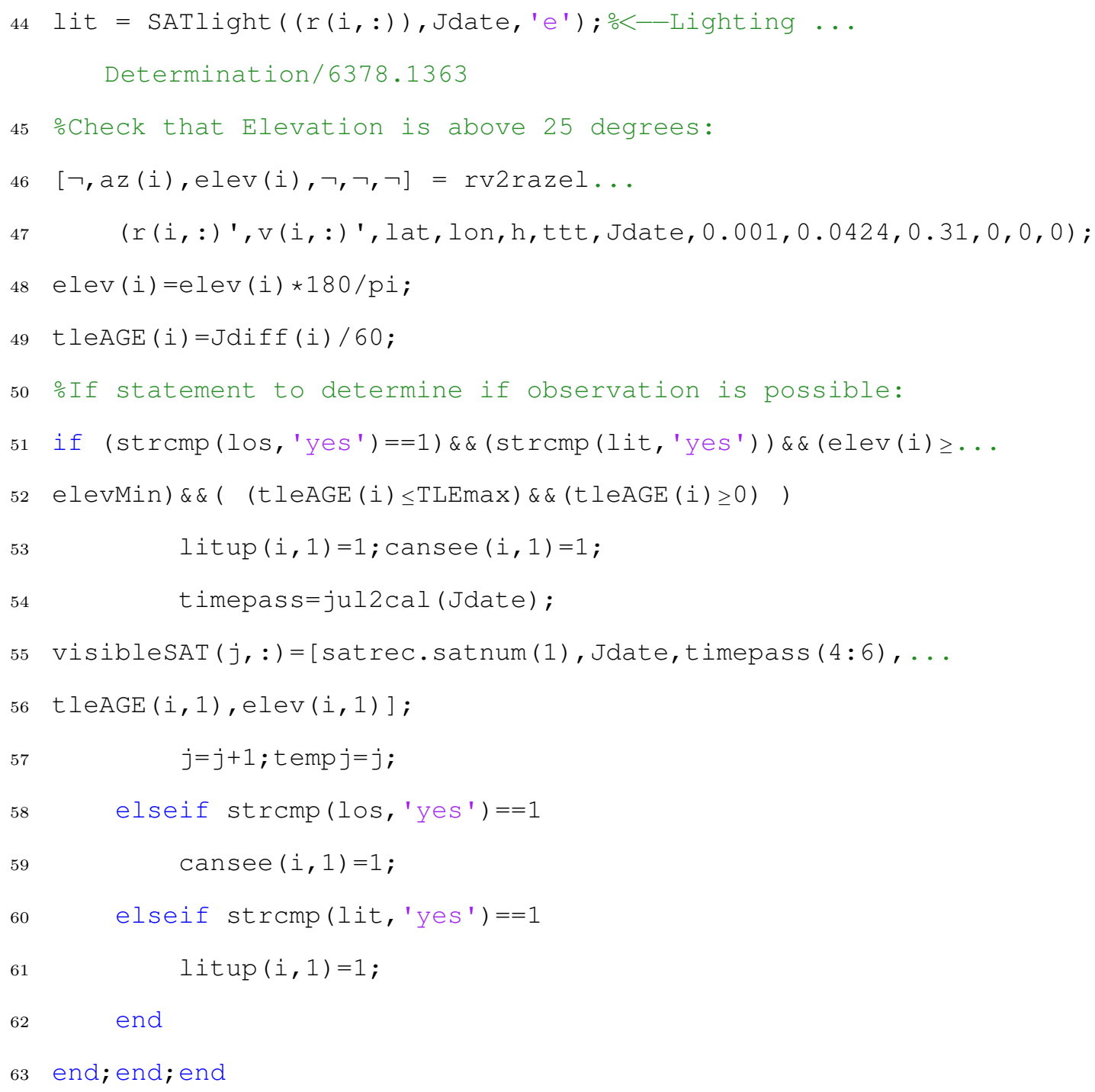

pixel_analysis.m

This code inputs observation data from images_pixelvals.csv, to be used in both data reduction and analysis, concerning orbit determination and temporal error growth.

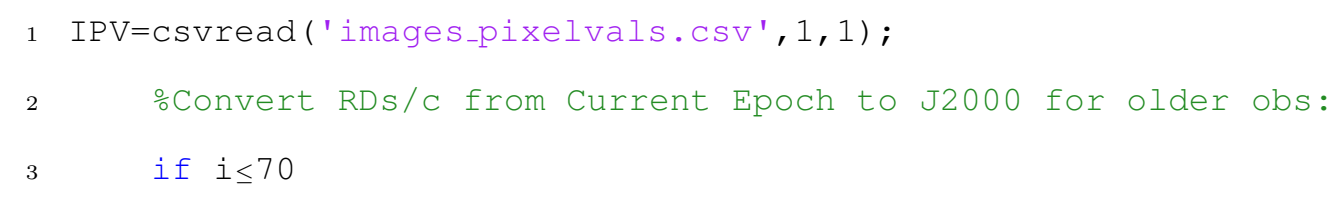




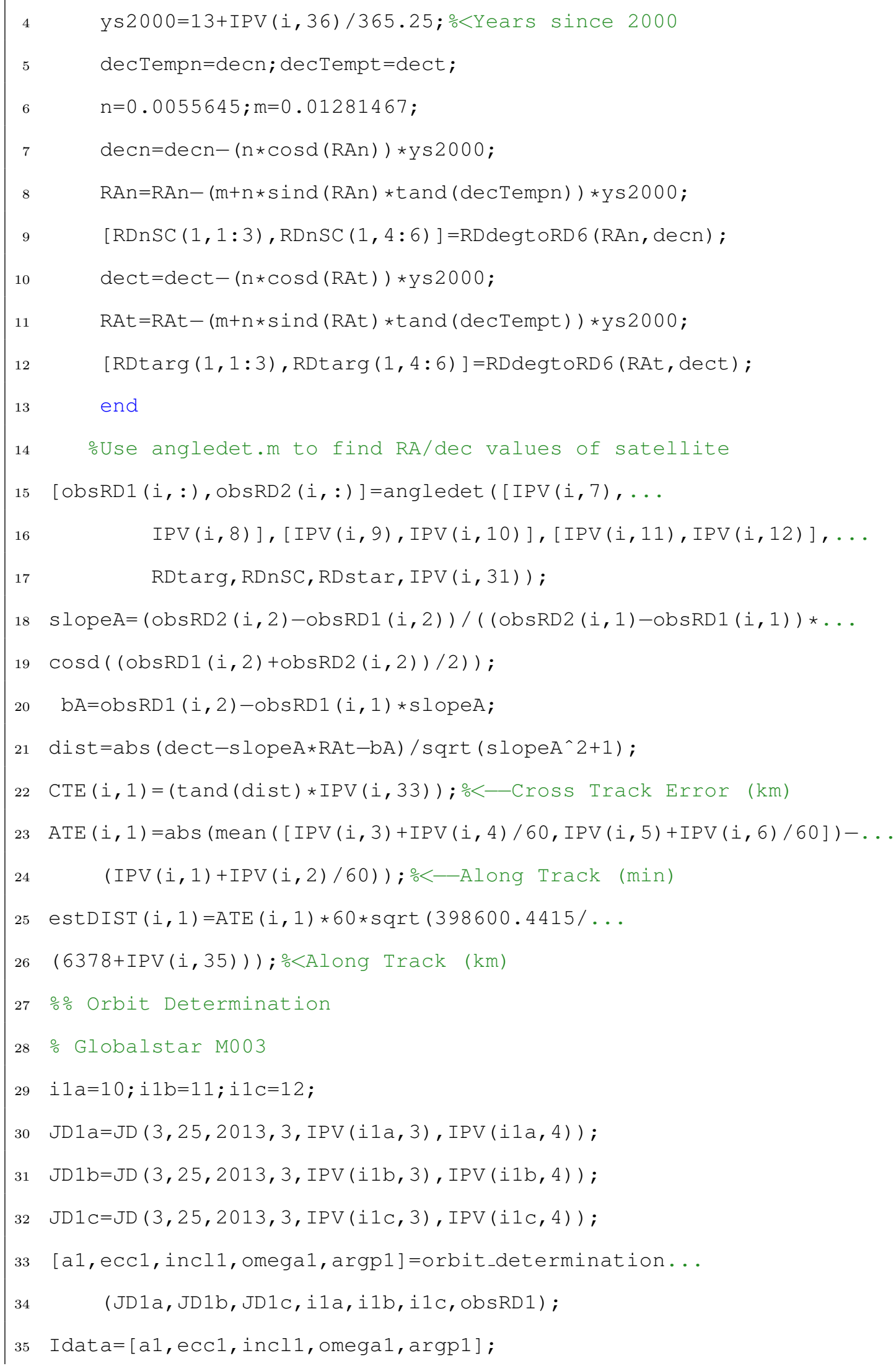


36 other_info=[JD1a, JD1b, JD1c, ila, i1b, ilc] ;

\section{RV2COE2.m}

This function converts Earth-centered inertial state vectors into classical orbital elements.

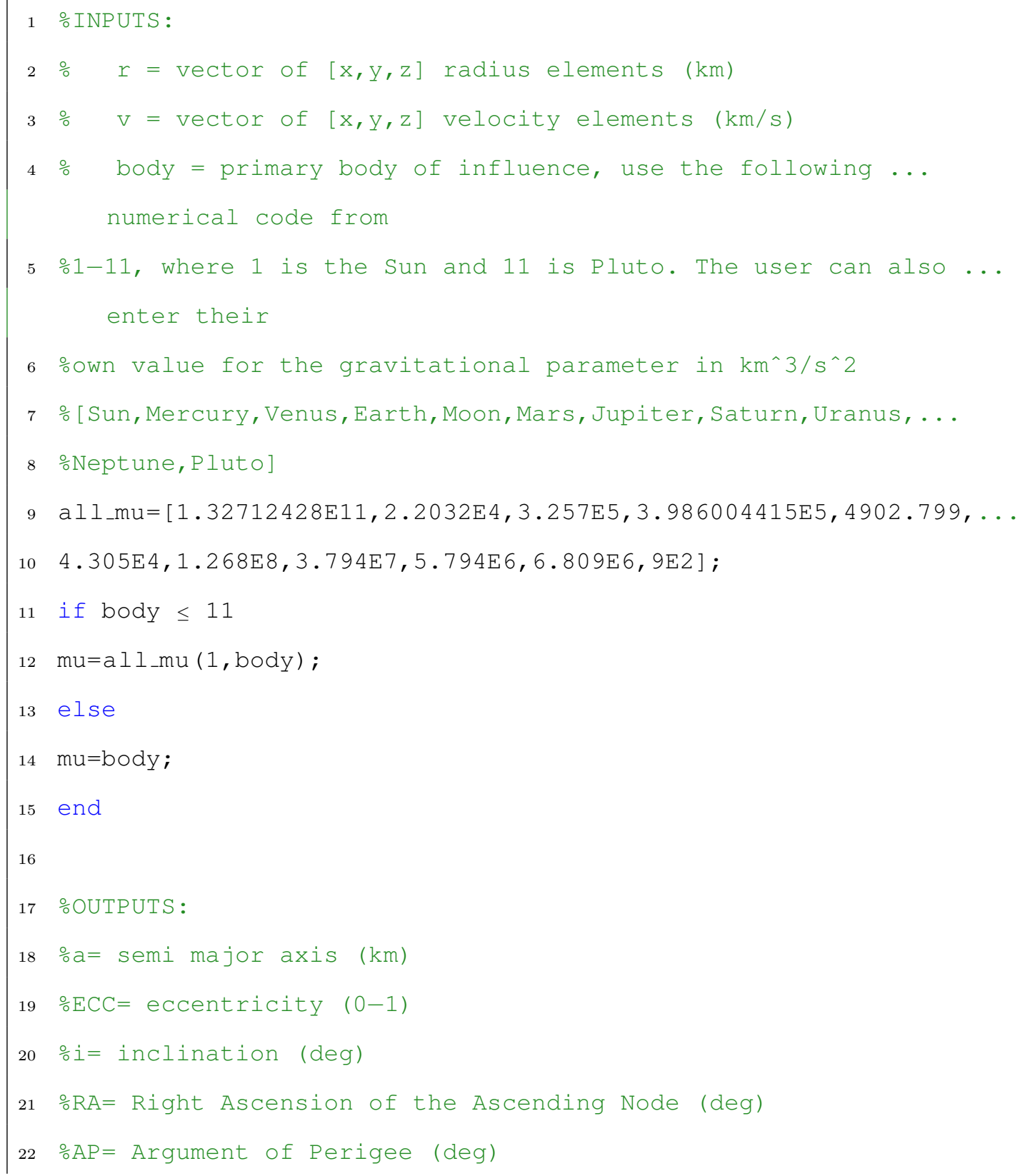




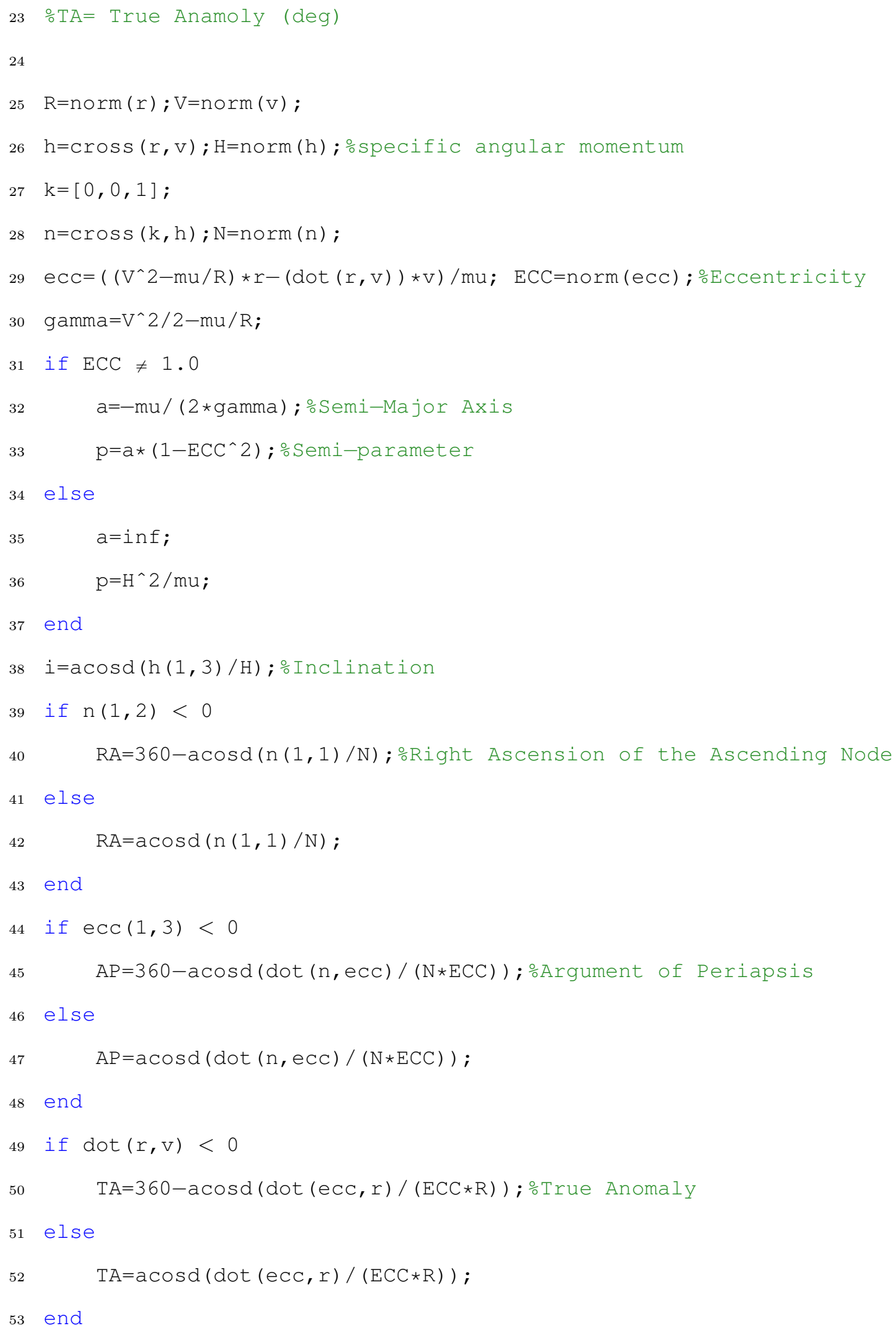




\section{STK2VIZIER.m}

This file converts the current Azimuth Elevation angles from STK into Right Ascension and Declination angles. It then writes them into a space-delimited file for uploading to Vizier.

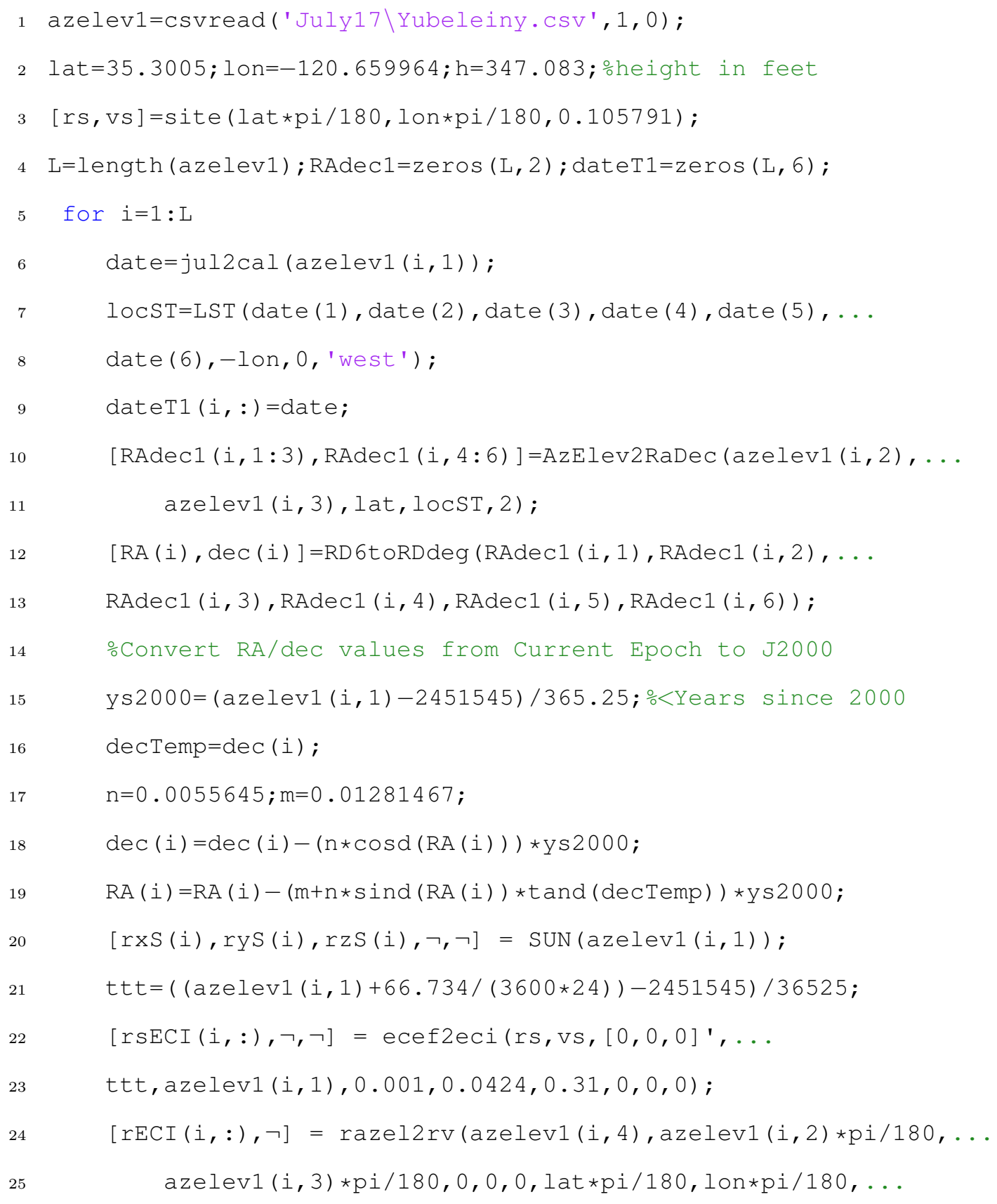




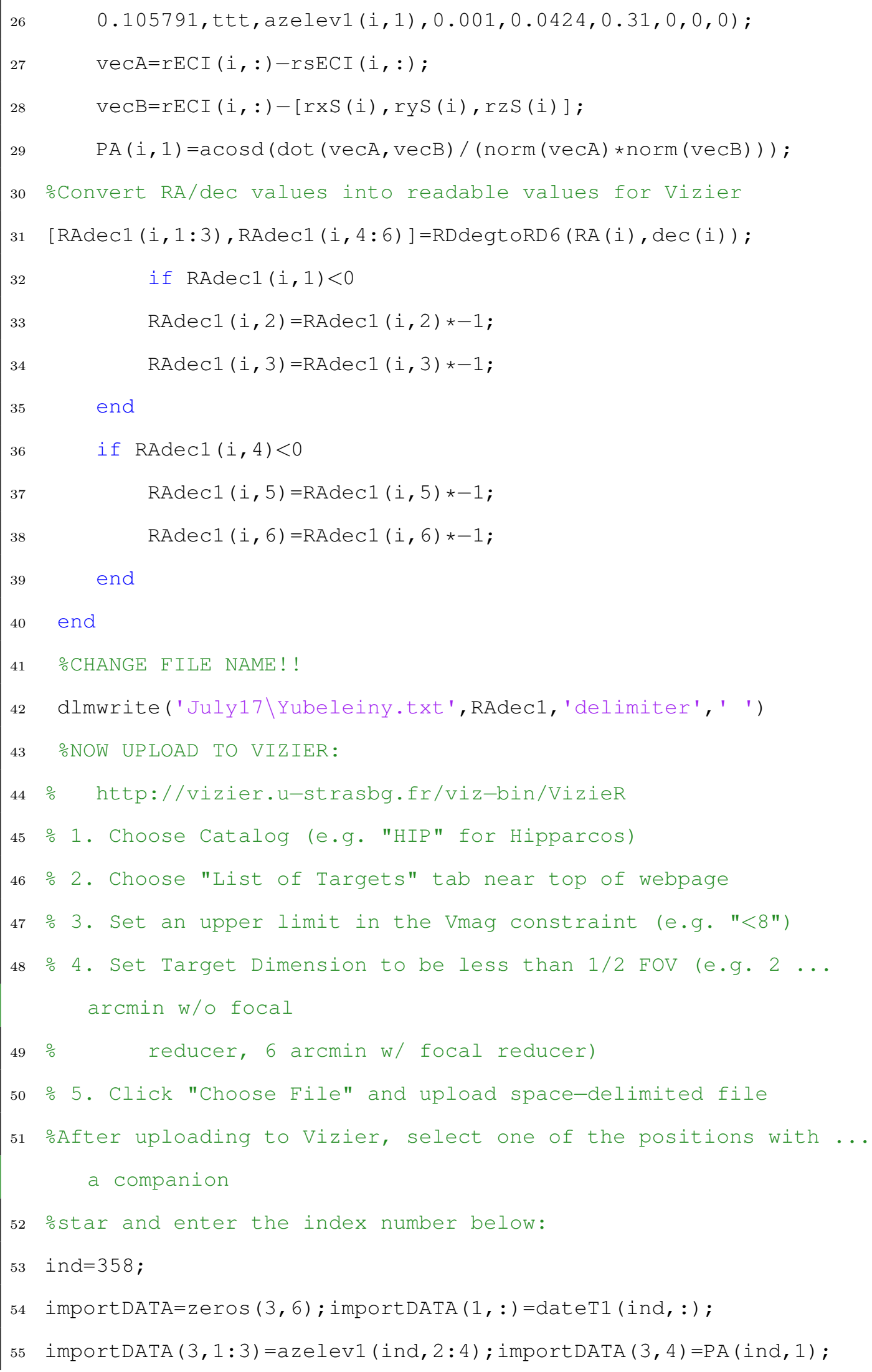


56 importdAtA $(2,:)=\operatorname{RAdec} 1($ ind, : ) ; 


\subsection{Extended Kalman Filter}

In the field of orbit determination, it is often the case that multiple sets of observation angles are gathered for a single satellite. These angle-sets are acquired from multiple overhead passes of the satellite, can be separated by days, and enable differential correction techniques such as least squares and Kalman filtering. This appendix will present a short summary of the technique used in this report, an extended Kalman filter, covered more thoroughly in Vallado's Fundamentals of Astrodynamics and Applications[29].

To enable the use of a Kalman filter, it is first necessary to define initial characteristics of the system: initial covariance, $P$, initial states, $X_{0}$, second moment of process noise, $Q$, and the measurement noise, $R$. Results from Appendix 7.6 are used to estimate the measurement noise,

$$
\begin{array}{r}
B=\left[\begin{array}{l}
0.0033^{\circ} \\
0.0038^{\circ}
\end{array}\right] \\
R=\left[\begin{array}{cc}
\frac{1}{B(1)^{2}} & 0 \\
0 & \frac{1}{B(2)^{2}}
\end{array}\right]
\end{array}
$$

where $b$ are the sensor biases involved in angle determination. The initial covariance and process noise matrices were estimated to be composed of the following values,

$$
P_{0}=\left[\begin{array}{cccccc}
1 k m^{2} & 0 & 0 & 0 & 0 & 0 \\
0 & 1 k m^{2} & 0 & 0 & 0 & 0 \\
0 & 0 & 1 k m^{2} & 0 & 0 & 0 \\
0 & 0 & 0 & 0.0001 \mathrm{~km}^{2} / \mathrm{s}^{2} & 0 & 0 \\
0 & 0 & 0 & 0 & 0.0001 \mathrm{~km}^{2} / \mathrm{s}^{2} & 0 \\
0 & 0 & 0 & 0 & 0 & 0.0001 \mathrm{~km}^{2} / \mathrm{s}^{2}
\end{array}\right]
$$


and process noise errors were determined in the following manner,

$$
v=\left[\begin{array}{c}
1 \mathrm{~km} \\
1 \mathrm{~km} \\
1 \mathrm{~km} \\
0.01 \mathrm{~km} / \mathrm{s} \\
0.01 \mathrm{~km} / \mathrm{s} \\
0.01 \mathrm{~km} / \mathrm{s}
\end{array}\right]
$$

where $v$ is the process noise. The accuracy of these estimations is unknown, and future effort could be spent on better quantifying these initial values.

Right ascension and declination observations, $z$, are inputted into the system, and the Kalman filter begins with the determination of the observation partial derivative, $H$, after which the error state-transition matrix, $\Phi$, is calculated (see Vallado 10.7.1),

$$
\begin{gathered}
X=\left[\begin{array}{l}
r_{x} \\
r_{y} \\
r_{z} \\
v_{x} \\
v_{y} \\
v_{z}
\end{array}\right] \\
z=\left[\begin{array}{l}
\alpha \\
\delta
\end{array}\right] \\
H=\frac{\partial z}{\partial X} \\
\Phi=\frac{\partial X}{\partial X_{0}}
\end{gathered}
$$


Although these matrices can be approximated either analytically or numerically, this project only used the analytical method of finite, central differencing to determine the partials. An algorithm form of a finite differencing technique can also be found in Vallado section 10.4.1. Kepler's universal variable propagator is used to propagate forward the current state, $X$, to the predicted state, $\hat{X}$, from which the MATLAB code topocentric.m is used to convert the states to topocentric right ascension and declination. The filter continues by predicting the subsequent error covariance,

$$
\bar{P}=\Phi P \Phi^{T}+Q
$$

From this point on, the filter determines the residuals, $b$, Kalman gain, $K$, and state error estimate, $\delta x$, and proceeds to update the error covariance and state. These steps directly follow those found in Algorithm 68 of Vallado,

$$
\begin{array}{r}
b=z-H \bar{X} \\
K=\bar{P} H^{T}\left[H \bar{P} H^{T}+R\right]^{-1} \\
\delta x=K b \\
P=\bar{P}-K H \bar{P} \\
X=\bar{X}+\delta x
\end{array}
$$


Table 7.6: Pixel Selection Errors determined by Monte-Carlo Method.

\begin{tabular}{|c|c|c|c|}
\hline Target Satellite & RA Error $\left(^{\circ}\right)$ & Dec Error $\left({ }^{\circ}\right)$ & CTE Error $(\mathrm{km})$ \\
\hline Globalstar M003 & $4.16 \mathrm{E}-03$ & $1.45 \mathrm{E}-03$ & 0.078 \\
$3 / 25$ & $2.19 \mathrm{E}-02$ & $3.95 \mathrm{E}-02$ & 0.637 \\
& $1.64 \mathrm{E}-03$ & $2.12 \mathrm{E}-03$ & 0.091 \\
\hline Globalstar M031 & $3.08 \mathrm{E}-03$ & $2.62 \mathrm{E}-03$ & 0.066 \\
$4 / 14$ & $3.81 \mathrm{E}-03$ & $3.95 \mathrm{E}-03$ & 0.052 \\
& $1.76 \mathrm{E}-03$ & $1.41 \mathrm{E}-03$ & 0.071 \\
\hline Globalstar M036 & $2.65 \mathrm{E}-03$ & $3.61 \mathrm{E}-03$ & 0.166 \\
$4 / 20$ & $2.62 \mathrm{E}-03$ & $1.64 \mathrm{E}-03$ & 0.104 \\
& $1.65 \mathrm{E}-03$ & $1.55 \mathrm{E}-03$ & 0.071 \\
\hline
\end{tabular}

\subsection{Error Analysis}

Error propagation was performed for single-pass orbit determination, crosstrack error, and apparent magnitude. Analysis of along-track error was omitted due to the unknown uncertainty in the computer clock error. Astrometrical uncertainties included pixel selection errors, \pm 1 pixel for star positions and \pm 2 pixels for satellite positions, and $\pm 0.004 \mathrm{arcsec} /$ pix for plate scale. Time stamp errors of $\pm 1 \mathrm{~ms}$ and a guessed computer clock error of $\pm 1 \mathrm{~s}$ were used as well ${ }^{5}$. The effect of these uncertainties were analyzed through the use of a 10,000 iteration Monte-Carlo method. Nine angle-sets were used as a sample (three single-trio observations) and $3 \sigma$ errors were generated from each-these are reported in Table 7.6. For orbit determination, these errors are reported in Tables 5.1 and 5.2 . As for cross-track error, a maximum uncertainty was set to the $3 \sigma$ value of the calculated errors - this uncertainty was calculated to be $0.558 \mathrm{~km}$ and is displayed as error bars in Fig 5.2.

\footnotetext{
${ }^{5}$ Although a computer clock error was used here, it was found that the uncertainties in along-track error were disproportionately affected when compared to the uncertainties in the cross-track error and orbit determination results. This is due to the fact that whatever clock error existed, was added equally to each observation. Therefore it was decided that while little harm would come from applying a general clock error elsewhere in the report (the magnitude of the resulting uncertainty was lower than that due to the pixel-selection errors), the effect of such an error upon the along-track error would not reflect the true uncertainties.
} 


\section{BIBLIOGRAPHY}

[1] M. R. Ackermann, J. T. McGraw, J. B. Martin, and P. C. Zimmer. Blind Search for Micro Satellites in LEO: Optical Signatures and Search Strategies. In AMOS Technical Conference, Sandia National Laboratories, Albuquerque, NM, September 2003.

[2] Adrian Ashford and David Tytell. SkyandTelescope.com's Scope Calculator. http://www.skyandtelescope.com/observing/objects/javascript/8875112.html, 2013.

[3] D. S. Birney, G. Gonzalez, and D. Oesper. Observational Astronomy. Cambridge University Press, New York, second edition, 2006.

[4] Centre de donnes astronomiques de Strasbourg. VizieR Service. http://vizier.u-strasbg.fr/viz-bin/VizieR, November 2011.

[5] Centre de donnes astronomiques de Strasbourg. Simbad Service. http://simbad.u-strasbg.fr/simbad/sim-fbasic, July 2013.

[6] Chaos Software Group, Inc. Atomic Clock Sync: a free configuration utility. http://www.worldtimeserver.com/atomic-clock/, 2013.

[7] H. Cowardin, G. Ojakangas, M. Mulrooney, S. Lederer, , and J. Liou. Optical Signature Analysis of Tumbling Rocket Bodies via Laboratory Measurements. AMOS, 2012.

[8] H. D. Curtis. Orbital Mechanics for Engineering Students. Elsevier, Burlington, MA, second edition, 2010. 
[9] Ed Grayzeck and E. Bell. NASA National Space Science Data Center. http://nssdc.gsfc.nasa.gov/nmc/, July 2013.

[10] M. E. Graff. Development of a Remotely Operated Autonomous Satellite Tracking System. M.S. Thesis, Air Force Institute of Technology, WrightPatterson Air Force Base, Ohio, March 2010.

[11] Hyperphysics Georgia State University. Ecliptic plane. http://hyperphysics.phy-astr.gsu.edu/hbase/Eclip.html, June 2009.

[12] James R. Hansen, NASA History Program Office. The Odyssey of Project Echo. http://history.nasa.gov/SP-4308/ch6.htm, 1994.

[13] D. J. Kessler, N. L. Johnson, J. Liou, and M. Matney. The Kessler Syndrome: Implications to Future Space Operations. In 33rd Annual AAS Guidance and Control Conference, volume 10, Breckenridge, Colorado, February 2010. AAS.

[14] A. Mammano. Observations of artificial satellites. In International Technical Scientific Meeting on Space, number 19-23, Rome, Italy, 1962.

[15] J. Mason. Development of a MATLAB/STK TLE Accuracy Assessment Tool, in Support of the NASA Ames Space Traffic Management Project. M.S. Thesis, International Space University, Moffet Field, CA, August 2009.

[16] A. Milani and G. F. Gronchi. Theory of Orbit Determination. Cambridge University Press, New York, 2010.

[17] H. Miles. Artificial Satellite Observing and its Applications. Faber and Faber Limited, 3 Queen Square, London, 1974.

[18] M. K. Mulrooney, M. J. Matney, , and E. S. Barker. A New Bond Albedo 
for Performing Orbital Debris Brightness to Size Transformations. In $I A C$ 08.A6.2.7, 2008.

[19] N2YO ITPROSTAR. Real Time Satellite Tracking. http://www.n2yo.com/, 2013.

[20] NASA Orbital Debris Program Office. Orbital Debris Graphics. http://orbitaldebris.jsc.nasa.gov/photogallery/beehives.html, October 2012.

[21] F. Patat. Observing During Bright Time: Tips and Tricks. ESO, 2004.

[22] PolySat. Cp5. http://polysat.calpoly.edu/launched-missions/cp5/, 2013.

[23] T. Schildknecht, U. Hugentohler, and A. Verdum. Optical Observations of Space Debris with the Zimmerwal 1-Meter Telescope. Advances in Space Research, 19(2):221-228, 1997.

[24] M. M. Schmunk. Initial Determination of Low Earth Orbits Using Commercial Telescopes. M.S. Thesis, Air Force Institute of Technology, WrightPatterson Air Force Base, Ohio, March 2008.

[25] Space Track Satellite Situation Report, JFCC SPACE, Vandenberg AFB, CA. https://www.spacetrack.org/basicspacedata/query/class/boxscore.html, Accessed 6/25/2013.

[26] M. L. Thrall. Orbit Determination of Highly Eccentric Orbits Using a Raven Telescope. M.S. Thesis, Naval Postgraduate School, Monterey, California, September 2005.

[27] T.S. Kelso. Space track tle retreiver version 2.0.1.1. http://celestrak.com/SpaceTrack/TLERetriever2Help.asp, April 2011. 
[28] T.S. Kelso and David Vallado . Astrodynamics Software, Fundamentals of Astrodynamics and Applications. http://www.celestrak.com/software/vallado-sw.asp, August 2012.

[29] D. A. Vallado. Fundamentals of Astrodynamics and Applications. Microcosm Press/Springer, Hawthorne, CA/New York, NY, third edition, 2007.

[30] D. A. Vallado, P. Crawford, R. Hujsak, and T. Kelso. Revisiting Spacetrack Report \#3. In AIAA/AAS Astrodynamics Specialist Conference, Keystone, CO, August 2006.

[31] D. A. Vallado and D. Finklman. A Critical Assessment of Satellite Drag and Atmospheric Density Modeling. In AAS/AIAA Astrodynamics Specialist Conference, Honolulu, HI, 2008.

[32] D. A. Vallado and J. D. Griesbach. Simulating Space Surveillance Networks. AAS, 11(580), Aug 2011. 